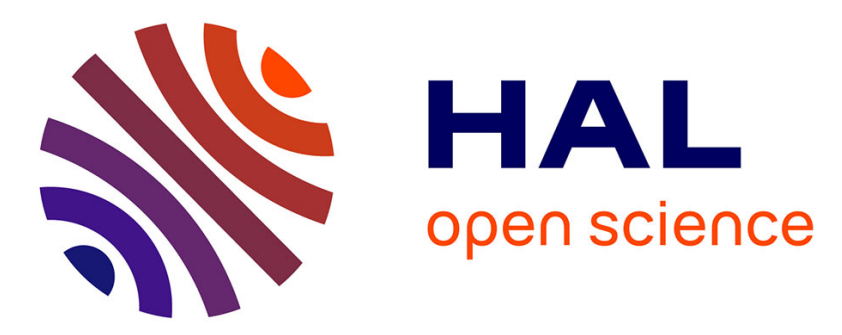

\title{
C 0 -rigidity of Lagrangian submanifolds and punctured holomorphic discs in the cotangent bundle
}

\author{
Cedric Membrez, Emmanuel Opshtein
}

\section{To cite this version:}

Cedric Membrez, Emmanuel Opshtein. C 0 -rigidity of Lagrangian submanifolds and punctured holomorphic discs in the cotangent bundle. Compositio Mathematica, 2021, 157 (11), pp.2433-2493. 10.1112/S0010437X21007570 . hal-03432683

\section{HAL Id: hal-03432683 \\ https://hal.science/hal-03432683}

Submitted on 17 Nov 2021

HAL is a multi-disciplinary open access archive for the deposit and dissemination of scientific research documents, whether they are published or not. The documents may come from teaching and research institutions in France or abroad, or from public or private research centers.
L'archive ouverte pluridisciplinaire HAL, est destinée au dépôt et à la diffusion de documents scientifiques de niveau recherche, publiés ou non, émanant des établissements d'enseignement et de recherche français ou étrangers, des laboratoires publics ou privés. 


\title{
$\mathcal{C}^{0}$-rigidity of Lagrangian submanifolds and punctured holomorphic discs in the cotangent bundle
}

\author{
Cedric Membrez* and Emmanuel Opshtein
}

May 25, 2021

\begin{abstract}
Our main result is the $\mathcal{C}^{0}$-rigidity of the area spectrum and the Maslov class of Lagrangian submanifolds. This relies on the existence of punctured pseudoholomorphic discs in cotangent bundles with boundary on the zero section, whose boundaries represent any integral homology class. We discuss further applications of these punctured discs in symplectic geometry.
\end{abstract}

\section{Introduction}

The main concern of this paper is a $\mathcal{C}^{0}$-rigidity result for the area spectrum and Maslov class of Lagrangian submanifolds. It can be stated in the language of $\mathcal{C}^{0}$-symplectic geometry. We recall the following definition due to Eliashberg-Gromov:

Definition. A symplectic homeomorphism $h:(M, \omega) \rightarrow\left(M^{\prime}, \omega^{\prime}\right)$ is a homeomorphism which is a $\mathcal{C}^{0}$-limit of symplectic diffeomorphisms.

The Eliashberg-Gromov $\mathcal{C}^{0}$-rigidity Theorem shows that a smooth symplectic homeomorphism is a symplectic diffeomorphism. This definition raises the question of determining how close symplectic homeomorphisms are to their smooth cousins. In [BO16] it was suggested to study this question from the perspective of the action of symplectic homeomorphisms on submanifolds.

Question 1. Let $N \subset(M, \omega)$ be a submanifold and assume that its image by a symplectic homeomorphism is a smooth submanifold $N^{\prime} \subset\left(M^{\prime}, \omega^{\prime}\right)$. Is $N^{\prime}$ symplectomorphic to $N$ ? Which classical symplectic invariants must coincide for $N$ and $N^{\prime}$ ?

The question is far from having an easy and definitive answer as demonstrated by the collection of results showing $\mathcal{C}^{0}$-rigidity (e.g. [LS94, Ops09, HLS15, HLS16]) or in contrast $\mathcal{C}^{0}$-flexibilty (e.g. [BO16, BHS16]). In the large the picture suggested by these works is that $\mathcal{C}^{0}$-rigidity prevails for coisotropic submanifolds (although several of their invariants have not yet undergone investigation), while it fails completely for most others. For instance in [BO16] a symplectic homeomorphism of $\mathbb{R}^{6}$ is constructed that maps an open symplectic

* Partially supported by the Swiss National Science Foundation grant 155540 and the European Research Council Advanced grant 338809 
disk to another smooth symplectic disk with half the symplectic area. On the other hand, from [LS94] we know that if $L \subset M$ is a closed Lagrangian submanifold and $h(L)$ is smooth for a symplectic homeomorphism $h$, then $h(L)$ is Lagrangian. Our main result is that in this situation the area spectrum and the Maslov class of $L$ and $h(L)$ coincide. More precisely, given a Lagrangian submanifold $L \subset(M, \omega)$, define its area homomorphism

$$
\begin{aligned}
\mathcal{A}_{\omega}^{L}: H_{2}(M, L) & \longrightarrow \mathbb{R} \\
\sigma & \longmapsto \int_{\Sigma} \omega, \text { where }[\Sigma]=\sigma,
\end{aligned}
$$

and its Maslov index $\mu_{L}: \pi_{2}(M, L) \rightarrow \mathbb{Z}$ (see $\S 4.1$ for the definitions of Maslov indices).

Theorem 1. Let $h:(M, \omega) \rightarrow\left(M^{\prime}, \omega^{\prime}\right)$ be a symplectic homeomorphism that sends a closed Lagrangian submanifold $L$ to a smooth (hence Lagrangian) submanifold $L^{\prime}$. Then

a) $\mathcal{A}_{\omega^{\prime}}^{L^{\prime}}=h_{*} \mathcal{A}_{\omega}^{L}$

b) $\mu_{L^{\prime}}=h_{*} \mu_{L}$.

It is not hard to see that theorem 1.a) provides a positive answer to question 1 for Lagrangian submanifolds, when the Nearby Lagrangian conjecture holds for $T^{*} L$ (see proposition 4.2). This conjecture is known to hold in $T^{*} \mathbb{T}^{2}$ [DRGI16], so symplectic homeomorphisms act on 2-dimensional tori in the same way as symplectic diffeomorphisms do. For instance, a symplectic homeomorphism of $\mathbb{C P}^{2}$ cannot take the Chekanov torus to the Clifford torus. The $\mathcal{C}^{0}$-rigidity of the area homomorphism of Lagrangian tori has already been proved in [BO16], but even follows from a theorem by Benci-Sikorav (see [Sik89]).

For the sake of completeness we briefly discuss an alternative approach to theorem 1. This approach differs from the one proposed in this paper and relies on deep results by Abouzaid and Abouzaid-Kragh on Lagrangian submanifolds in cotangent bundles [Abo11, Kra11], but thus far a complete proof has not been written explicitly in the literature. Given a closed manifold $L$ endowed with a Riemannian metric $g$, we define $\ell_{g}^{\min }(\beta)$ to be the length of the minimizing geodesic in a class $\beta \in H_{1}(L ; \mathbb{Z})$. In the cotangent bundle $\pi: T^{*} L \rightarrow L$ endowed with its standard symplectic structure $d \lambda=d(p d q)$ we also define

$$
\mathcal{W}(L, g, r):=\left\{\|p\|_{g}<r\right\}:=\left\{(q, p) \in T^{*} L \mid\|p\|_{g_{q}}<r\right\} \subset T^{*} L
$$

where $\|\cdot\|_{g_{q}}$ is the natural dual norm on $T_{q}^{*} L$. Let $L^{\prime} \subset \mathcal{W}(L, g, \varepsilon)$ be a Lagrangian submanifold whose projection to the zero-section induces an isomorphism in the homology of degree 1. In this context, Kragh-Abouzaid proved on one hand in [Kra11, Appendix E] that

$$
\mu_{L^{\prime}}\left(\beta^{\prime}\right)=0, \quad \forall \beta^{\prime} \in H_{1}\left(L^{\prime}\right),
$$

and Abouzaid computed on the other hand the wrapped Floer homology of $L^{\prime}$ with a fiber of the cotangent bundle [Abo11]. This computation allows for constructing a graph selector for $L^{\prime}$ [AOO16], similar to the one obtained in [PPS03] via generating functions for those $L^{\prime}$ which are Hamiltonian isotopic to the zero-section. This graph selector can be used as in [PPS03] to show that

$$
\lambda\left(\beta^{\prime}\right) \leq \varepsilon \ell_{g}^{\min }\left(\pi_{*} \beta^{\prime}\right) \quad \forall \beta^{\prime} \in H_{1}\left(L^{\prime}\right)
$$


By the Weinstein neighbourhood theorem, these local results imply that if $L, L^{\prime}$ are two Lagrangian submanifolds of a symplectic manifold $M$ satisfying the assumptions of theorem 1 , the area homomorphisms of $L$ and $L^{\prime}$ are $\varepsilon$-close and their Maslov classes coincide, which in turn implies theorem 1 . This approach puts the $\mathcal{C}^{0}$-rigidity of these symplectic invariants in the framework of Abouzaid's work on wrapped Floer homology and Fukaya categories.

In the present paper we choose to explain another approach, that lies more within the classical framework of pseudoholomorphic techniques in symplectic geometry. The advantage is twofold. Apart from providing an easier and totally self-contained proof of theorem 1 , the technical tool on which it relies is a new existence result for certain punctured holomorphic curves in cotangent bundles, that proves very useful. The following result is the technical core of our paper.

Theorem 2. Let $(L, g)$ be a closed Riemannian manifold and $\beta \in H_{1}(L ; \mathbb{Z})$. Assume that $g$ is generic, in the sense that it has a unique minimizing geodesic $\gamma(\beta)$ in class $\beta$. Then, for every almost complex structure $J$ on $T^{*} L$ compatible with $d \lambda$ and $g$-cylindrical at infinity, there exists a J-holomorphic punctured disc $u:(D \backslash\{0\}, \partial D) \rightarrow\left(T^{*} L, L\right)$ asymptotic to a lift of $\gamma(\beta)$ at 0 .

See the begining of section 3 , and in particular definition 3.1 for a complete description of $g$-cylindrical almost complex structures. This theorem has several applications. Some of them, e.g. theorem 1 or theorem 5.2, can be obtained by arguments based on existing techniques such as wrapped Floer homology [Abo11] or symplectic homology [AS06, SW06], but theorem 2 provides a unified way of looking at these results. For instance, we recover (1) and (2) under stronger assumptions, which turn out to be enough for the proof of theorem 1.

Theorem 3. Let $(L, g)$ and $\left(L^{\prime}, g^{\prime}\right)$ be two closed Riemannian manifolds and $\iota: L^{\prime} \hookrightarrow$ $\left(T^{*} L, d \lambda\right)$ a Lagrangian embedding such that $\pi_{*} \circ \iota_{*}: H_{1}\left(L^{\prime}, \mathbb{Z}\right) \rightarrow H_{1}(L, \mathbb{Z})$ is an isomorphism. Assume that $\iota$ extends to a symplectic embedding $\mathcal{I}$ of a neighborhood of $L^{\prime}$ such that

$$
L \subset \mathcal{I}\left(\mathcal{W}\left(L^{\prime}, g^{\prime}, \varepsilon^{\prime}\right)\right) \subset \mathcal{W}(L, g, \varepsilon) \subset T^{*} L
$$

for some $\varepsilon, \varepsilon^{\prime}>0$. Then for all $\beta^{\prime} \in H_{1}\left(L^{\prime} ; \mathbb{Z}\right)$ we have

a) $\left|\iota^{*} \lambda\left(\beta^{\prime}\right)\right| \leq \varepsilon \ell_{g}^{\min }\left(\pi_{*} \circ \iota_{*} \beta^{\prime}\right)$,

b) $\mu_{\iota\left(L^{\prime}\right)}\left(\iota_{*} \beta^{\prime}\right)=0$.

On the other hand, these holomorphic curves also have further applications, like the computation of some Poisson bracket invariants that can be used to bound the size of Weinstein neighbourhoods of Lagrangian submanifolds. We briefly elaborate further on this application. In [EGM16], invariants of tuples $(L, \pi, M)$ were constructed, where $L$ is a Lagrangian torus of $M$ with a fibration $\pi: L \rightarrow S^{1}$. We generalize this invariant to arbitrary Lagrangian submanifolds, replacing $\pi$ by any cohomology class $a$ of degree 1 . We denote this invariant by $b p(L, a, M)$ (see section 5.2). Theorem 2 allows an exact computation of this invariant in cotangent bundles. 
Theorem 4. Let $(L, g)$ be a closed Riemannian manifold and $\mathcal{W}(L, g, r):=\left\{\|p\|_{g}<r\right\} \subset$ $T^{*} L$. Let also $a \in H^{1}(L ; \mathbb{Z})$ be a primitive class. Then we have

$$
b p(L, a, \mathcal{W}(L, g, r))=\frac{r}{\|a\|_{\mathrm{st}}}=r \inf \left\{\frac{\ell_{g}(\gamma)}{a([\gamma])} \mid \gamma \in \mathcal{C}^{1}\left(S^{1}, L\right), a([\gamma])>0\right\} .
$$

The invariants $b p(L, a, M)$ being monotone in $M$, the previous theorem allows us to bound the size of Weinstein neighbourhoods of Lagrangian submanifolds. We illustrate this idea with the example of the Clifford torus in $\mathbb{C P}^{2}$ (see section 5.2).

We wish to make a final remark in this introduction. The problem of $\mathcal{C}^{0}$-flexibility/rigidity of submanifolds was introduced in [Ops09, HLS15, BO16]. At least for the second author of the present paper, the formalization of these questions in the framework of $\mathcal{C}^{0}$-symplectic geometry came with the hope of starting a study of a new type of geometry. Ideally, the $\mathcal{C}^{0}$-rigid properties of $\mathcal{C}^{0}$-rigid submanifolds (e.g. the coisotropic ones) would provide interesting invariants. For instance, the fact that the characteristic foliation of a hypersurface is $\mathcal{C}^{0}$-rigid [Ops09] led to conjecturing that topological hypersurfaces in a symplectic manifold are covered by "characteristic sets", which would be invariant under symplectic homeomorphisms. In [HLS15], the concept of a $\mathcal{C}^{0}$-Lagrangian submanifold was introduced: these are topological $n$-dimensional submanifolds of symplectic manifolds which can be locally straightened via symplectic homeomorphisms to $\mathbb{R}^{n} \subset \mathbb{C}^{n}$. In this perspective theorem 1 raises the question whether $\mathcal{C}^{0}$-Lagrangian submanifolds can be equipped with an area homomorphism invariant by $\mathcal{C}^{0}$-symplectic homeomorphisms. This is however not the case, starting from dimension 6. Let indeed $L:=\partial D \times \partial D \times \partial D \subset \mathbb{C}^{3}$, where $D$ is the closed Euclidean disc of radius 1 centered at 0 in $\mathbb{C}$. Then $D^{\prime}:=D \times\{1\} \times\{1\}$ is a disc of area $\pi$ attached to this Lagrangian submanifold. By [BO16] there exists a symplectic homeomorphism $h$ of $\mathbb{C}^{3}$ such that $h\left(D^{\prime}\right)=\frac{1}{2} D^{\prime}:=D\left(0, \frac{1}{2}\right) \times\{1\} \times\{1\}$. Then $h(L)$ is a $\mathcal{C}^{0}$-Lagrangian submanifold of $\mathbb{C}^{3}$, but the area of $h\left(D^{\prime}\right)$ does not coincide with the area of $D^{\prime}$. Even worse, since the homeomorphism $h$ can have support localized in an arbitrary neighbourhood of $D^{\prime}$, the area of smooth discs with boundary on $h(L)$ do not depend only on the relative homology class of these discs.

Organisation of the paper. In $\S 2$ we present yet another short proof of theorem 1 for Lagrangian tori based on holomorphic discs, which illustrates our motivation for establishing theorem 2. $\S 3$ is the technical core of the paper and provides the proof of theorem 2 . Although this result might not be a surprise to experts in the field, we have not been able to find details in the literature and we give a detailed account here. The Fredholm theory for punctured holomorphic discs in symplectic cobordisms with boundary on a Lagrangian submanifold is provided in appendix A for the sake of completeness. In $\S 4$ we establish our main applications, theorems 1 and 3 . In $\S 5$ we discuss further applications of theorem 2 like theorem 4.

Aknowledgements. E.O. wishes to thank Nicolas Vichery and Frol Zapolsky for their suggestion of using the work of Abouzaid to prove theorem 1. He also wishes to very warmly thank Charles Boubel for many discussions on Riemannian geometry. Part of this work was realized during a visit of E.O. to the Technion and Tel Aviv University in fall 
2014. He thanks Misha Entov and Lev Buhovski for the invitation and for many stimulating discussions, in particular on $p b_{4}$-type invariants, which ultimately resulted in the present proof of theorem 1. C.M. would like to thank Paul Biran and Leonid Polterovich for enlightening discussions and stimulating questions related to the work. Both authors would like to thank Chris Wendl very warmly for help on issues of transversality for punctured holomorphic disks.

Notation. We work in the smooth category. Unless explicitly stated, all our manifolds and their structures are smooth. We adopt the following notation in this paper. It will be regularly recalled in the course of the paper. Let $(L, g)$ be a closed Riemannian manifold.

- $L \subset\left(T^{*} L, d \lambda\right)$ denotes the zero section of the cotangent bundle with its standard Liouville form $\lambda$ and $L$ is a Lagrangian submanifold.

- $T_{r}^{*} L=\mathcal{W}(L, g, r):=\left\{(q, p) \in T^{*} L \mid\|p\|_{g}<r\right\}$ and $\mathcal{W}_{g}:=\mathcal{W}(L, g, 1)=\{(q, p) \in$ $\left.T^{*} L \mid\|p\|_{g}<1\right\}$ is the unit (co)disk bundle.

- $M:=\partial \mathcal{W}_{g}$ is the contact boundary of $\mathcal{W}_{g}$ with the contact form $\alpha:=\lambda_{\mid M}$.

- $\gamma:[0, \ell] \rightarrow L$ is a geodesic with unit speed with respect to $g$ and $\ell_{g}(\gamma)=\ell$ is the length of $\gamma$.

- $\ell_{g}^{\min }(\beta)$ is the minimal length of a closed connected curve in a class $\beta \in H_{1}(L ; \mathbb{Z})$. We call a closed geodesic with this length a minimizing geodesic in class $\beta$.

- $\tilde{\gamma}:[0, \ell] \rightarrow M$ is the lift of a unit speed geodesic $\gamma$ to $M$ given by $\tilde{\gamma}(t)=(\gamma(t), g(\dot{\gamma}(t), \cdot))$. Note that

$$
\int_{\tilde{\gamma}} \alpha=\ell_{g}(\gamma)=\ell .
$$

- $D=\{z \in \mathbb{C}|| z \mid \leq 1\}$ is the closed unit disk and $\partial D=\{z \in \mathbb{C}|| z \mid=1\} . D(z, R)$ denotes the closed disk of radius $R$ centered at $z$.

- int $\Omega$ denotes the interior of a set $\Omega$.

- $\mathrm{Op}(X, Y)$ or $\mathrm{Op}(X)$ is an open neighborhood of $X \subset Y$.

\section{A source of motivation for theorem 2}

This section is independent from the rest of the paper. It aims at explaining at least one source of motivation for establishing theorem 2 (existence of punctured holomorphic disks). We start the discussion with a proof of theorem 1.a) for tori, which we think illustrates our purpose well. As explained in [BO16, lemma 5.1] it follows directly from the following theorem.

Theorem 2.1. For $\varepsilon>0$ let $\iota: L \hookrightarrow\left(S^{1} \times(-\varepsilon, \varepsilon)\right)^{n} \subset\left(T^{*} \mathbb{T}^{n}, d \lambda\right)$ be a Lagrangian embedding such that $\pi \circ \iota: L \rightarrow \mathbb{T}^{n}$ induces an isomorphism in homology. Let $e_{j}:=$ $(\pi \circ \iota)^{*}\left(\left[0_{\mathbb{T}^{j-1}} \times S^{1} \times 0_{\mathbb{T}^{n-j}}\right]\right) \in H_{1}(L), j=1, \ldots, n$. Then

$$
\left|\iota^{*} \lambda\left(e_{j}\right)\right| \leq \varepsilon
$$


Proof: We prove that $\left|\iota^{*} \lambda\left(e_{1}\right)\right| \leq \varepsilon$. Without loss of generality we can assume that $\iota^{*} \lambda\left(e_{1}\right) \geq$ 0 and $\varepsilon<1$. Put $\iota^{*} \lambda\left(e_{j}\right)=\varepsilon \Delta_{j}$ with $\Delta_{j} \in \mathbb{R}$ and $\Delta_{1} \in[0, \infty)$. Denote by $\left\lfloor\Delta_{1}\right\rfloor \in \mathbb{N}$ the integer part and by $\operatorname{Frac}\left(\Delta_{1}\right):=\Delta_{1}-\left\lfloor\Delta_{1}\right\rfloor \in[0,1)$ its fractional part. Define

$$
\begin{aligned}
\kappa & :=1+\varepsilon\left\lfloor\Delta_{1}\right\rfloor, \\
a_{1} & :=1-\varepsilon \operatorname{Frac}\left(\Delta_{1}\right)>0, \\
a_{j} & :=N \kappa-\varepsilon \Delta_{j},
\end{aligned}
$$

with $N$ chosen large enough such that $a_{j}>a_{1}>0$ and $a_{j}+\varepsilon \Delta_{j} \geq 2 \kappa$ for $j=2, \ldots, n$. Consider now the standard symplectic embedding $\Phi:\left(\left(S^{1} \times(-\varepsilon, \varepsilon)\right)^{n}, d \lambda\right) \hookrightarrow\left(\mathbb{C}^{n}, \omega_{\text {st }}\right)$ with $\Phi\left(0_{\mathbb{T}^{n}}\right)=S^{1}\left(a_{1}\right) \times \cdots \times S^{1}\left(a_{n}\right) \subset \mathbb{C}^{n}\left(S^{1}(a) \subset \mathbb{C}\right.$ being the circle that encloses a disk of area $a$ ). Then $\mathcal{W}:=\operatorname{Im} \Phi=\left\{\bigcup S^{1}\left(t_{1}\right) \times \ldots \times S^{1}\left(t_{n}\right)|| t_{i}-a_{i} \mid<\varepsilon\right\}$. Obviously,

$$
e_{d}(\mathcal{W}) \leq a_{1}+\varepsilon \leq 1+\varepsilon
$$

and equality in the second inequality can hold only if $a_{1}=1$, i.e. $\Delta_{1} \in \mathbb{N}\left(e_{d}\right.$ denotes the displacement energy in $\left.\left(\mathbb{C}^{n}, \omega_{\mathrm{st}}\right)\right)$. On the other hand, the discs with boundaries on $\Phi \circ \iota(L)$ representing the classes $e_{1}, \ldots, e_{n}$ have symplectic areas $a_{1}+\varepsilon \Delta_{1}=1+\varepsilon\left\lfloor\Delta_{1}\right\rfloor=\kappa$,

$a_{2}+\varepsilon \Delta_{2}=N \kappa, \ldots, a_{n}+\varepsilon \Delta_{n}=N \kappa$. Since $\pi \circ \iota$ is an isomorphism in homology, we therefore see that every disc with boundary on $\Phi \circ \iota(L)$ and positive area has area equal to a multiple of $\kappa$. By a theorem of Chekanov [Che98], we infer that

$$
1+\varepsilon\left\lfloor\Delta_{1}\right\rfloor=\kappa \leq e_{d}(\Phi \circ \iota(L)) \leq e_{d}(\mathcal{W}) \leq 1+\varepsilon
$$

We conclude that $\varepsilon\left\lfloor\Delta_{1}\right\rfloor \leq \varepsilon$, so $\Delta_{1} \leq 1$, with equality only if $\Delta_{1} \in \mathbb{N}$. Thus $\iota^{*} \lambda\left(e_{1}\right) \leq \varepsilon$. Applying the same procedure to the classes $e_{2}, \ldots, e_{n}$ we obtain the statement of the theorem.

What makes this proof easy (although it ultimately relies on a deep theorem by Chekanov [Che98]), is that we have a very large sample of Lagrangian embeddings of tori in $\mathbb{C}^{n}$ with holomorphic discs of arbitrary relative areas, so that we can choose which is responsible for the displacement energy of the Lagrangian submanifold. This situation is unfortunately very specific to tori: there are very few examples of Lagrangian embeddings of a given manifold into a symplectic manifold, even without speaking of the freedom to choose the area of holomorphic discs with boundary on this submanifold. On the other hand, a manifold always embeds into its cotangent bundle as the zero section, which is Lagrangian. In this setting there are no symplectic discs with boundary on the zero section, but punctured holomorphic discs may replace the compact discs with a similar benefit.

\section{Punctured holomorphic curves in cotangent bundles}

Let $L$ be a closed manifold and $\left(T^{*} L \stackrel{\pi}{\rightarrow} L, \lambda\right)$ be its cotangent bundle equipped with the Liouville form $\lambda=p d q$. The cotangent bundle is endowed with an $\mathbb{R}_{*}^{+}$-action given by $\tau \cdot(q, p):=(q, \tau p)$. Let $g$ be a Riemannian metric on $L$ and $\beta \in H_{1}(L ; \mathbb{Z})$. If $g$ is generic, 
$\beta$ has exactly one connected representative of minimal length, hence a geodesic, whose arc-length parametrization is henceforth denoted by $\gamma(\beta)$ (or even $\gamma$ when there is no risk of confusion). For general $g$ there may be several connected minimal representatives and $\gamma(\beta)$ is then one of them. We give a short proof of these claims. Let $\beta \in H_{1}(L)$. By a standard theorem (see e.g. [Cha06, Theorem IV.5.1]), every non-trivial free homotopy class in a compact Riemannian manifold has a minimal closed geodesic. Free homotopy classes are in a one-to-one correspondence with conjugacy classes of the fundamental group $\pi_{1}(L)$. Now since the Hurewicz homomorphism $\pi_{1}(L) \rightarrow H_{1}(L)$ is surjective and $H_{1}(L)$ is abelian, we get a well-defined surjective map from the set of conjugacy classes of $\pi_{1}(L)$ to $H_{1}(L)$. Thus there is at least one closed and connected geodesic representing the class $\beta$, but also possibly many, whose lengths form a subset $\Lambda(\beta)$ of $\mathbb{R}^{+}$. Consider now a sequence of constant speed geodesic loops $\gamma_{n}: S^{1} \rightarrow L$ representing $\beta$ whose lengths tend to the infimum of $\Lambda(\beta)$. By Arzela-Ascoli Theorem a subsequence converges uniformly to a loop $\gamma: S^{1} \rightarrow L$, whose length equals inf $\Lambda(\beta)$ by semi-continuity of the length functional in the uniform topology. This loop is then automatically a geodesic (else there would be a geodesic with even shorter length in the same free homotopy class by the theorem mentioned just above), with minimal length among the connected geodesic loops representing the class $\beta$. Note that, by [Abr70, Ano82], for generic Riemannian metrics the length spectrum is discrete and for any closed geodesic only its time-reversal has the same length. This proves that there is a unique connected minimal geodesic representing $\beta$ in the generic case.

The metric $g$ induces an isomorphism $\sharp: T L \rightarrow T^{*} L, v \mapsto v^{\sharp}=g(v, \cdot)$, hence an inner product (still denoted $g$ ) and a norm $\|\cdot\|_{g}$ on the fibers of $T^{*} L$. We write for the unit disk bundle and its boundary

$$
\mathcal{W}_{g}:=\mathcal{W}(L, g, 1):=\left\{(q, p) \mid q \in L, p \in T_{q}^{*} L,\|p\|_{g}<1\right\} \text { and } M:=\partial \mathcal{W}_{g}
$$

Then $\alpha:=\lambda_{\mid M}$ is a contact form on $M$, whose Reeb vector field is denoted by $R$. It is well-known that $R$ generates the cogeodesic flow on $M$ : its trajectories are the lifts $\tilde{\gamma}(t):=\left(\gamma(t), \dot{\gamma}(t)^{\sharp}\right)$ of the unit speed geodesics $\gamma$. Moreover, $\left(T^{*} L, d \lambda\right)$ is a symplectic cobordism with one positive end given by the identification:

$$
([1, \infty) \times M, r \alpha) \stackrel{\sim}{\longrightarrow}\left(T^{*} L \backslash \mathcal{W}_{g}, \lambda\right), \quad(r,(q, p)) \longmapsto(q, r p) .
$$

Note that this map extends to $((0, \infty) \times M, r \alpha) \simeq\left(T^{*} L \backslash L, \lambda\right)$. The function $r=\|p\|_{g} \in$ $[0, \infty)$ is called the radial coordinate in $T^{*} L$. The image of the vector field $r \frac{\partial}{\partial r}$ under this identification is Liouville.

An almost complex structure $J$ on $T^{*} L$ is compatible with $\omega:=d \lambda$ if $\omega(\cdot, J \cdot)$ is a positive definite quadratic form. An almost complex structure $J$ on $(0, \infty) \times M$ is compatible with $\alpha$ if $J$ preserves ker $\alpha_{\mid\{r\} \times M}$ and is compatible with $d \alpha=\omega$ on this subbundle, sends $r \frac{\partial}{\partial r}$ to the Reeb vector field $R$ of $(\{r\} \times M, \alpha)$ and is invariant by the $\mathbb{R}_{*}^{+}$-action on $(0, \infty) \times M$. Hence a metric $g$ on $L$ defines on $T^{*} L$ a fiberwise convex hypersurface $M$, which in turn determines a 1-form $\alpha$, and in fine a class of almost complex structures on $(0, \infty) \times M \simeq T^{*} L \backslash L$, namely those which are compatible with $\alpha$. Let $\gamma$ be a closed geodesic with unit speed and length $\ell:=\ell_{g}(\gamma)$ and let $\tilde{\gamma}$ be its lift to $M$. Then for any almost complex structure $J$ compatible 
with $\alpha$ it can be shown that

$$
v_{\gamma, g}: \mathbb{R} \times \mathbb{R} / \ell \mathbb{Z} \longrightarrow(0, \infty) \times M, \quad(s, t) \longmapsto\left(e^{s}, \tilde{\gamma}(t)\right),
$$

is $J$-holomorphic for the complex structure $j \partial_{s}=\partial_{t}$ on $\mathbb{R} \times \mathbb{R} / \ell \mathbb{Z}$.

Definition 3.1. An almost complex structure $J$ on $T^{*} L$ compatible with $d \lambda$ is cylindrical at infinity (with respect to $g$ ) if it is compatible with $\alpha$ outside a compact neighbourhood of the zero section. We denote by $\mathcal{J}_{\mathrm{Cyl}, g}^{\infty}$ the set of all such almost complex structures, where the $\infty$ of the notation aims at reminding that these structures are constrained only near infinity. A J-holomorphic curve $u$ defined on $U \backslash\left\{z_{0}\right\} \subset \mathbb{C}$ (where $U$ is an open subset and $\left.z_{0} \in U\right)$ is said to be asymptotic to $\tilde{\gamma}$ at $z_{0}$ if there exists a biholomorphic identification of a punctured neighborhood of $z_{0}$ with $[0, \infty) \times \mathbb{R} / \ell \mathbb{Z}$ that provides coordinates $(s, t)$ such that $d\left(u(s, t), v_{\gamma, g}(s, t)\right) \underset{s \rightarrow \infty}{\longrightarrow} 0$. Here, $d$ stands for any distance which is $\mathbb{R}_{*}^{+}$-invariant on the cylindrical end.

The aim of this section is to prove the following result. We say that a metric $g$ has discrete length spectrum in the class $\beta$ if the lengths of the geodesics representing this class form a discrete set.

Theorem 3.2. Let $(L, g)$ be a closed Riemannian manifold, $\beta \in H_{1}(L ; \mathbb{Z})$ a homology class in which $g$ has discrete length spectrum and $\tilde{\gamma}(\beta)$ be the lift of a unit-speed connected geodesic of minimal length in class $\beta$. Then for all $J \in \mathcal{J}_{\mathrm{Cyl}, g}^{\infty}$ there exists a map $u_{J}:(D \backslash\{0\}, \partial D) \rightarrow$ $\left(T^{*} L, L\right)$ which solves the following problem:

$$
\left\{\begin{array}{l}
d u_{J} \circ j=J\left(u_{J}\right) \circ d u_{J}, \\
u_{J *}[\partial D]=\beta, \\
u_{J} \text { is asymptotic to } \tilde{\gamma}(\beta) \text { at } 0 .
\end{array}\right.
$$

In other terms the set $\widehat{\mathcal{M}}(J, \beta)$ of solutions of $\mathcal{P}(J, \beta)$ is non-empty for all $J \in \mathcal{J}_{\mathrm{Cyl}, g}^{\infty}$.

Remarks 3.3. (i) $S^{1}$ acts on $\widehat{\mathcal{M}}(J, \beta)$ by holomorphic source reparametrizations (of $D \backslash\{0\})$. We denote in the sequel $\mathcal{M}(J, \beta):=\widehat{\mathcal{M}}(J, \beta)_{/ S^{1}}$, the space of unparametrized solutions of $\mathcal{P}(J, \beta)$.

(ii) As already mentioned, when a metric has several minimizing geodesics in the class $\beta$, $\gamma(\beta)$ stands for some minimizing geodesic.

(iii) If the length spectrum is not discrete, the proof of theorem 3.2 will provide for all $\varepsilon>0$ a map $u_{J, \varepsilon}$ asymptotic to a lift of a geodesic in the class $\beta$ of length at most $\ell_{g}(\gamma(\beta))+\varepsilon$.

(iv) Theorem 3.2 also holds in homotopy. Namely, if $\beta$ is a free homotopy class, and $g$ satifies the assumptions of the theorem, then for all $J \in \mathcal{J}_{\mathrm{Cyl}, g}^{\infty}$ there exists a punctured $J$-holomorphic disc asymptotic at the puncture to the minimal geodesic in the class $\beta$. 
The scheme of proof of theorem 3.2 is mostly standard and we explain it in the present paragraph, under the assumption that $\gamma(\beta)$ is a primitive geodesic, i.e. not a multiplecover. Let $g$ be a metric with discrete length spectrum in the class $\beta$, and assume that $\gamma(\beta)$ is primitive. We first define an almost complex structure $J_{h} \in \mathcal{J}_{\mathrm{Cyl}, h}^{\infty}$ for an arbitrary metric $h$ on $L$ (§3.1) which has a unique $J_{h}$-holomorphic punctured disc $u_{\gamma, h} \in \widehat{\mathcal{M}}\left(J_{h}, \beta\right)$ modulo reparametrization ( $(3.2)$. We also prove that all elements of $\mathcal{M}(J, \beta)$ for $J \in \mathcal{J}_{\mathrm{Cyl}, h}^{\infty}$ are somewhere injective $(\S 3.5)$. For a suitable perturbation $g_{\varepsilon}$ of our metric $g$ we prove the surjectivity of the linearization of the $\bar{\partial}_{J_{g_{\varepsilon}}}$-operator at $u_{\gamma, g_{\varepsilon}}(\S 3.3$ and $\S 3.4)$. In $\S 3.6$ we show compactness: if a sequence of almost complex structures $J_{n} \in \mathcal{J}_{\mathrm{Cyl}, g_{\varepsilon}}^{\infty}$ coincide with $J_{g_{\varepsilon}}$ outside a compact set and converge to $J \in \mathcal{J}_{\mathrm{Cyl}, g_{\varepsilon}}^{\infty}$ in the $\mathcal{C}^{\infty}$-topology, then a sequence $u_{n}$ of solutions of $\mathcal{P}\left(J_{n}, \beta\right)$ has a subsequence that converges to a solution of $\mathcal{P}(J, \beta)$. Classical arguments then show that if $\left\{J_{t}\right\}_{t \in[0,1]}$ is a smooth generic path of almost complex structures from $J_{g_{\varepsilon}}$ to a generic $J_{n} \in \mathcal{J}_{\mathrm{Cyl}, g_{\varepsilon}}^{\infty}$ that coincide with $J_{g_{\varepsilon}}$ outside a compact set, the set $\cup\{t\} \times \mathcal{M}\left(J_{t}, \beta\right)$ is a one-dimensional compact cobordism between the point $\{0\} \times \mathcal{M}\left(J_{g_{\varepsilon}}, \beta\right)$ and $\{1\} \times \mathcal{M}\left(J_{n}, \beta\right)$, hence the latter space is non-empty. Applying again compactness to a generic sequence $J_{n}$ of approximations of $J$ that coincide with $J_{g_{\varepsilon}}$ outside a compact set, we find a solution of $\mathcal{P}(J, \beta)$ for all $J \in \mathcal{J}_{\mathrm{Cyl}, g_{\varepsilon}}^{\infty}$ that coincide with $J_{g_{\varepsilon}}$ at infinity. We finally use an SFT-compactness argument to obtain the theorem for the metric $g$ itself $(\S 3.7)$.

\subsection{A particular almost complex structure}

Let $g$ be an arbitrary Riemannian metric on $L$. In this section we describe a particular almost complex structure on $T^{*} L$ that is determined by the metric $g$.

The Levi-Civita connection $\nabla$ of $g$ induces a natural connection $\nabla^{*}$ on $T^{*} L$ by the identity

$$
d(\sigma(X))=\nabla^{*} \sigma(X)+\sigma(\nabla X)
$$

for any 1-form $\sigma$ and vector field $X$ on $L$. This connection provides a splitting $T\left(T^{*} L\right)=$ $H \oplus F$, where $H$ is the horizontal distribution of $\nabla^{*}$ and $F$ is the distribution of the tangent spaces to the fibers. Note that we have a canonical identification $F_{(q, p)} \simeq T_{q}^{*} L$. We call $\pi: T^{*} L \rightarrow L$ the natural projection and, by abuse of notation, $\pi: H_{(q, p)} \rightarrow T_{q} L$ the isomorphism $d \pi_{\mid H}$, at least when there is no risk of confusion. In order to define our almost complex structure we first need to explain some relations between the Riemannian geometry on $L$ and the contact structure on $M$. Recall that $R$ denotes the Reeb vector field on $M$, hence defines a vector field on $(0, \infty) \times M$, independant of the first coordinate. We also see it as a vector field on $T^{*} L \backslash L \simeq(0, \infty) \times M$ via the isomorphism that extends (3). Notice then that $R$ is $\mathbb{R}_{*}^{+}$-invariant on $T^{*} L \backslash L$. The vector field $r \frac{\partial}{\partial r}=p$ is the infinitesimal generator of the $\mathbb{R}_{*}^{+}$-action on $T^{*} L \backslash L$. Also notice that $g$ induces an inner-product on $T_{q}^{*} L$, still denoted $g$, by $g_{q}\left(u^{\sharp}, v^{\sharp}\right):=g_{q}(u, v)$ for $u, v \in T_{q} L$. By definition $M=\left\{(q, p) \mid g_{q}(p, p)=1\right\}$.

Lemma 3.4. On $T^{*} L \backslash L \simeq(0, \infty) \times M$ we have

$$
\text { 1. } \left.R(q, p) \in H_{(q, p)} \text { and } g_{q}(\pi(R(q, p)), \cdot)=\frac{p}{\|p\|_{g}}=\frac{\partial}{\partial r} \text { (in other terms, } \pi(R)^{\sharp}=\frac{\partial}{\partial r}\right) \text {. }
$$


2. $H$ is a subspace of $T(\{r\} \times M)$ for every $r \in(0, \infty)$ and is Lagrangian with respect to $d \lambda$.

3. The map $\sharp \circ \pi$ is an isomorphism between $\operatorname{ker} \alpha \cap H_{(q, p)}$ and $\operatorname{ker} \alpha \cap F_{(q, p)}$. Moreover,

$$
\begin{aligned}
\operatorname{ker} \alpha \cap H_{(q, p)} & =\left\{V \in H_{(q, p)} \mid p(\pi(V))=0\right\} \\
\operatorname{ker} \alpha \cap F_{(q, p)} & =\left\{V \in F_{(q, p)} \mid g_{q}(V, p)=0\right\} .
\end{aligned}
$$

4. $\operatorname{ker} \alpha=\left(\operatorname{ker} \alpha \cap H_{(q, p)}\right) \oplus\left(\operatorname{ker} \alpha \cap F_{(q, p)}\right)$.

Proof:

1. Let us recall that $R(p, q)$ generates the co-geodesic flow of speed 1 on $\{1\} \times M$, and is extended to $T^{*} L \backslash L$ by $\mathbb{R}_{*}^{+}$-invariance. So $R(q, p)$ is horizontal in $\{1\} \times M$, and since the horizontal distribution is also $\mathbb{R}_{*}^{+}$-invariant, $R(q, p) \in H_{(q, p)}$ for all $(q, p) \in T^{*} L$. Moreover, the $\mathbb{R}_{*}^{+}$-action is trivial in $q$ (meaning that $\pi(\tau \cdot(q, p))=q$ ), so

$$
\pi(R(q, p))=\pi\left(R\left(q, \frac{p}{\|p\|}\right)\right)=\left(\frac{p}{\|p\|}\right)^{\sharp} .
$$

It follows that $\pi(R)^{\sharp}=\frac{p}{\|p\|}=\frac{\partial}{\partial r}$.

2. $H \subset T(\{r\} \times M)$ because parallel transport preserves the metric. It is also Lagrangian since the Levi-Civita connection has no torsion [Gri98].

3. The fact that $\operatorname{ker} \alpha \cap H_{(q, p)}=\left\{V \in H_{(q, p)} \mid p(\pi(V))=0\right\}$ and $\operatorname{ker} \alpha \cap F_{(q, p)}=F_{(q, p)} \cap$ $T_{(q, p)} M=\left\{V \in F_{(q, p)} \mid g_{q}(V, p)=0\right\}$ are immediate by definition. Now let $V \in$ ker $\alpha \cap H_{(q, p)}$. Since $\sharp$ is an isometry by definition of $g_{q}$ on $T_{q}^{*} L$ and $p(\pi(V))=0$, we have the chain of equalities

$$
g_{q}\left(\pi(V)^{\sharp}, p\right)=\|p\|_{g} g_{q}\left(\pi(V)^{\sharp}, \pi(R)^{\sharp}\right)=\|p\|_{g} g_{q}(\pi(V), \pi(R))=p(\pi(V))=0,
$$

which means that $\pi(V)^{\sharp} \in \operatorname{ker} \alpha \cap F_{(q, p)}$. By dimension considerations we see that $\sharp \circ \pi$ is indeed an isomorphism.

4. This is obvious by dimension considerations, since by (3), we have $\operatorname{dim} \operatorname{ker} \alpha \cap F_{(q, p)}=$ $n-1=\operatorname{dim} \operatorname{ker} \alpha \cap H_{(q, p)}$.

We now choose a smooth non-decreasing function $\chi:(0, \infty) \rightarrow[1, \infty)$ with $\chi(r)=1$ near 0 and $\chi(r)=r$ near $\infty$. Lemma 3.4 implies that

$$
T_{(q, p)} T^{*} L=\left\langle\frac{\partial}{\partial r}, R(q, p)\right\rangle \oplus \operatorname{ker} \alpha \cap H_{(q, p)} \oplus \operatorname{ker} \alpha \cap F_{(q, p)} \quad \forall q \in L, p \neq 0 .
$$

We define the almost complex structure $J_{g}$ on $T^{*} L \backslash L$ by

$$
\left\{\begin{array}{cll}
J_{g}(R)=-\chi(r) \frac{\partial}{\partial r} & & \\
J_{g}: \operatorname{ker} \alpha \cap H_{(q, p)} & \longrightarrow & \operatorname{ker} \alpha \cap F_{(q, p)} \\
V & \longmapsto & -g_{q}(\pi(V), \cdot) \\
J_{g}^{2}=-\mathrm{Id} . & &
\end{array}\right.
$$


By lemma 3.4, $J_{g}$ indeed exchanges $\operatorname{ker} \alpha \cap H_{(q, p)}$ and $\operatorname{ker} \alpha \cap F_{(q, p)}:$ if $V \in \operatorname{ker} \alpha \cap H_{(q, p)}$, $-g_{q}\left(J_{g} V, p\right)=g_{q}\left(\pi(V)^{\sharp}, p\right)=\|p\|_{g} g_{q}\left(\pi(V)^{\sharp}, \pi(R)^{\sharp}\right)=\|p\|_{g} g_{q}(\pi(V), \pi(R))=p(\pi(V))=0$.

Since $\chi=1$ near 0 , the first point of lemma 3.4 shows that $J_{g}$ has the following alternative definition near the zero section: for $V \in H, J_{g} V=-g(\pi(V), \cdot) \in F$. This obviously shows that $J_{g}$ extends smoothly to the zero section, because $H_{(q, p)}$ varies smoothly with $(q, p)$ and equals $T_{q} L$ when $p=0$. On the zero section we have a canonical isomorphism $T\left(T^{*} L\right)_{\mid L} \simeq T L \oplus T^{*} L$ and $J_{g}=-\sharp$.

Lemma 3.5. $J_{g} \in \mathcal{J}_{\mathrm{Cyl}, g}^{\infty}$.

Proof: By definition, $J_{g} R(q, p)=-r \frac{\partial}{\partial r}$ when $\|p\|_{g} \gg 1$ and $J_{g}$ preserves ker $\alpha$. Since $H_{(q, p)}$ is Lagrangian, showing that $J_{g \mid \operatorname{ker} \alpha}(q, p)$ is compatible with $d \lambda(q, p)$ amounts to proving that $d \lambda\left(V, J_{g} V\right)>0$ for $V \in \operatorname{ker} \alpha \cap H_{(q, p)}$. Moreover, since $d \lambda$ is invariant by the lifts of diffeomorphisms of $L$ to $T^{*} L$, a straightforward computation shows that we can assume $g_{q}=\sum d q_{i}^{2}$. Then, for $V \in \operatorname{ker} \alpha \cap H_{(q, p)}$,

$$
\begin{array}{rlr}
d \lambda\left(V, J_{g} V\right) & =\sum d p_{i} \wedge d q_{i}\left(V, J_{g} V\right) & \\
& \left.=-\sum d p_{i}\left(J_{g} V\right) d q_{i}(\pi(V)) \quad \text { (because } J_{g} V \in F_{(q, p)}, \text { so } d q_{i}\left(J_{g} V\right)=0\right) \\
& =\sum d p_{i}(g(\pi(V), \cdot)) d q_{i}(\pi(V)) \\
& =\sum d q_{i}(\pi(V))^{2}>0 & \\
\text { (because } \left.g_{q}=\sum d q_{i}^{2}\right) .
\end{array}
$$

Finally, to show that $J_{g}$ is dilation-invariant at infinity, it is enough to work on $\operatorname{ker} \alpha \cap H$ (because $H=(\operatorname{ker} \alpha \cap H) \oplus\langle R\rangle)$. If $V \in \operatorname{ker} \alpha \cap H, J_{g} V \in F \cap T M$, so the dilations on $(0, \infty) \times M$ act by the identity on both $V$ and $J_{g} V$.

We will also need the following properties of the almost complex structure $J_{g}$.

Proposition 3.6. The automorphism of $T^{*} L$ defined by $\sigma(q, p):=(q,-p)$ is $J_{g}$-antiholomorphic. Moreover, the sub-levels $\left\{\|p\|_{g} \leq r\right\}$ are $J_{g}$-pseudoconvex domains for $r>0$.

Proof: We consider canonical coordinates $(q, p) \in T^{*} L$ determined by a local choice of coordinates $q=\left(q_{1}, \ldots, q_{n}\right)$ on $L$. One can show that the horizontal distribution $H_{(q, p)}$ of the connection $\nabla^{*}$ is spanned by the vectors

$$
{\frac{\partial}{\partial q_{i}}}^{H}=\frac{\partial}{\partial q_{i}}+\sum_{k, l} \Gamma_{i l}^{k}(q) p_{k} \frac{\partial}{\partial p_{l}}, \quad i=1, \ldots, n,
$$

where $\Gamma_{i l}^{k}$ are the Christoffel symbols of the metric $g$. Since $d \sigma \frac{\partial}{\partial p_{l}}=-\frac{\partial}{\partial p_{l}}$, we see that $\sigma$ preserves the lifts ${\frac{\partial}{\partial q_{i}}}^{H}$ and hence the horizontal distribution. A brief calculation then reveals that $\sigma$ is $J_{g}$-antiholomorphic.

In $T^{*} L \backslash L$ one easily sees that $J_{g}$ preserves the contact distribution ker $\alpha$ and is compatible with $d \alpha$, hence the sets $\left\{\|p\|_{g} \leq r\right\}$ are $J_{g}$-pseudoconvex for $r>0$. 


\section{$3.2 \quad$ Computation of $\mathcal{M}\left(J_{g}, \beta\right)$}

The almost complex structure $J_{g}$ sends $R$ to a multiple of the radial vector field $\frac{\partial}{\partial r}$, hence for any closed unit speed geodesic $\gamma: \mathbb{R} / \ell \mathbb{Z} \rightarrow L$ the image of the map $v_{\gamma, g}$ of (4) is $J_{g^{-}}$ holomorphic (its tangent planes are $J_{g}$-invariant). We now need a holomorphic parametrization of this set. Recall that

$$
J_{g} R=-\chi(r) \frac{\partial}{\partial r}
$$

where $\chi$ is an increasing, weakly convex function with $\chi(r)=1$ for $r$ close to 0 and $\chi(r)=r$ outside a compact set. Then,

$$
G(u):=\int_{0}^{u} \frac{d r}{\chi(r)}
$$

is a well-defined strictly increasing function $G:[0, \infty) \rightarrow[0, \infty)$ with $G(0)=0$. Its inverse defines a function $G^{-1}:[0, \infty) \rightarrow[0, \infty)$ that satisfies the differential equation $h^{\prime}=\chi \circ h$. A straightforward computation shows that

$$
u_{\gamma, g}:(0, \infty) \times \mathbb{R} / \ell \mathbb{Z} \longrightarrow(0, \infty) \times M, \quad(s, t) \longmapsto\left(G^{-1}(s), \tilde{\gamma}(t)\right)
$$

is a $J_{g}$-holomorphic parametrization of $\operatorname{Im} v_{\gamma, g}$. One can see that this map extends as a map from the half-cylinder $[0, \infty) \times \mathbb{R} / \ell \mathbb{Z}$ to $T^{*} L$, which we also call $u_{\gamma, g}$. The half-cylinder $([0, \infty) \times \mathbb{R} / \ell \mathbb{Z}, j)$ is conformally equivalent to the closed punctured disk $(D \backslash\{0\}, i)$ via the map $(s, t) \mapsto \exp (-2 \pi(s+i t) / \ell)$. In general we will use the half-cylinder and closed punctured disk interchangeably. Hence we can consider $u_{\gamma, g}:(D \backslash\{0\}, \partial D) \rightarrow\left(T^{*} L, L\right)$. It is asymptotic to $\tilde{\gamma}$ at the puncture and we have $u_{\gamma, g}\left(e^{-2 \pi i t / \ell}\right)=\gamma(t) \subset L \subset T^{*} L$. In other words, $u_{\gamma, g} \in \widehat{\mathcal{M}}\left(J_{g}, \beta\right)$. The aim of this section is to prove that $\mathcal{M}\left(J_{g}, \beta\right)$ consists of the unique element $\left\{\left[u_{\gamma, g}\right]\right\}$. We require some preliminary lemmas.

Lemma 3.7. Let $u:(D \backslash\{0\}, \partial D) \rightarrow\left(T^{*} L, L\right)$ be a $J_{g}$-holomorphic map asymptotic to $\tilde{\gamma}$ at 0 . Then

(i) $u$ is smooth up to $\partial D$,

(ii) $\operatorname{Crit}(u)$ is a finite set of $D \backslash\{0\}$,

(iii) $u^{-1}(L) \cap \operatorname{int} D \backslash\{0\}$ is countable and can only accumulate at critical points of $u$.

Proof: Point (i) is a general and classical fact that relies on the ellipticity of the $\bar{\partial}_{J_{g}}$-operator on the space of curves with boundary on totally real submanifolds (see also [IS10]). Points (ii) and (iii) are specific to our situation. They rely on the special form of $J_{g}$ that makes the involution $\sigma(q, p):=(q,-p)$ anti-holomorphic, see proposition 3.6.

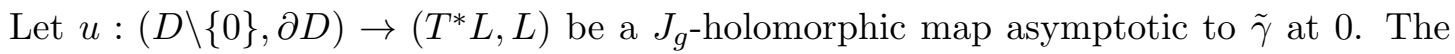
map $v: \mathbb{C} \backslash$ int $D \rightarrow T^{*} L$ defined by $v(z):=\sigma \circ u(1 / \bar{z})$ is $J_{g}$-holomorphic and coincides with $u$ on $\partial D$ (because $u(\partial D) \subset L=\operatorname{Fix}(\sigma))$. Thus, the map

$$
\begin{aligned}
w: \mathbb{C} \backslash\{0\} & \longrightarrow T^{*} L \\
z & \longmapsto \begin{cases}u(z) & \text { if } z \in D \backslash\{0\} \\
v(z) & \text { if } z \in \mathbb{C} \backslash D\end{cases}
\end{aligned}
$$


is holomorphic on $\mathbb{C} \backslash \partial D$, continuous on $\partial D$, and since $u$ and $v$ are smooth up to $\partial D$, w is $J_{g}$-holomorphic on $\partial D$ as well. Thus, $w: \mathbb{C} \backslash\{0\} \rightarrow T^{*} L$ is $J_{g}$-holomorphic. Since $w$ is asymptotic to $\tilde{\gamma}$ at 0 , it has no critical points near 0 and [MS12, Lemma 2.4.1] guarantees that $w$ has only finitely many critical points in $D \backslash\{0\}$, where it coincides with $u$. This proves (ii).

We prove (iii) by contradiction. First assume there exists a sequence $z_{n} \in \operatorname{int} D \backslash\{0\}$ that converges to a point $z \in \operatorname{int} D \backslash\{0\}$, which is not a critical point of $u$, and such that $u\left(z_{n}\right) \in L$ for all $n$ (hence $u(z) \in L$ ). We define

$$
\Omega:=\left\{\xi \in \operatorname{int} D \backslash\{0\} \mid \exists \xi^{\prime} \in \mathbb{C} \backslash D: u(\xi)=v\left(\xi^{\prime}\right)\right\} .
$$

In particular, $\Omega \supset u^{-1}(L) \cap \operatorname{int} D \backslash\{0\} \ni z_{n}$. By [MS12, Lemma 2.4.3], $\Omega$ contains a neighbourhood of each of its accumulation points in int $D \backslash\{0\}$ that are not critical points of $u$. It therefore contains a neighbourhood of $z$ by assumption. Notice that the asymptotic behaviour of $u$ at 0 implies that $\Omega$ does not meet some neighbourhood of the puncture 0 . Let now $c:[0,1] \rightarrow D$ be a continuous curve between $c(0)=z$ and $c(1)=0$ that avoids the (finitely many) critical points of $u$. Let $t_{*}:=\sup \{t \mid c([0, t]) \subset \Omega\}$. Since $z \in$ int $\Omega$, and $c(\mathrm{Op}(1)) \cap \Omega=\emptyset$, we have $0<t_{*}<1$. Let now $t_{n}<t_{*}$ be any sequence that converges to $t_{*}$. By assumption $c\left(t_{n}\right) \in \Omega$, so there exists $\xi_{n}^{\prime} \in \mathbb{C} \backslash D$ such that $v\left(\xi_{n}^{\prime}\right)=u\left(c\left(t_{n}\right)\right)$. Obviously $\xi_{n}^{\prime}$ cannot accumulate at $\infty$ because $v\left(\xi_{n}^{\prime}\right)=u\left(c\left(t_{n}\right)\right) \rightarrow u\left(c\left(t_{*}\right)\right) \in T^{*} L$. It can neither accumulate at a point $\xi^{\prime} \in \mathbb{C} \backslash D$ because then, by [MS12, Lemma 2.4.3], $c\left(t_{*}\right)$ would be an accumulation point of $\Omega$, so would be contained in int $\Omega$. Thus $c(t)$ would belong to $\Omega$ for $t<t_{*}+\varepsilon, \varepsilon>0$, which contradicts our definition of $t_{*}$. We therefore conclude that, after extraction, $\xi_{n}^{\prime} \rightarrow \xi^{\prime} \in \partial D$, so $u\left(c\left(t_{*}\right)\right)=\lim u\left(c\left(t_{n}\right)\right)=\lim v\left(\xi_{n}^{\prime}\right)=v\left(\xi^{\prime}\right) \in v(\partial D) \subset L$. This shows that any curve that joins $z$ to 0 in $D \backslash \operatorname{Crit}(u)$ intersects $u^{-1}(L)$. Since $\operatorname{Crit}(u)$ is finite, we conclude that the connected component $U$ of $z$ in $D \backslash u^{-1}(L)$ does not contain 0 . Thus $u_{\mid U}:(U, \partial U) \rightarrow\left(T^{*} L, L\right)$ is $J_{g}$-holomorphic. If $\partial U$ is smooth, we can conclude by Stokes Theorem. In general, we can invoke the pseudoconvexity of the hypersurfaces $\left\{\|p\|_{g}=r\right\}$ for $r>0$ (see proposition 3.6) to conclude that $u_{\mid U}$ takes values in $L$. Since $U$ is open and $L$ is totally real, we conclude that $u_{\mid U}$ is constant, which implies that $u$ is constant. But this contradicts the fact that $u$ is asymptotic to $\tilde{\gamma}$ at 0 .

This contradiction shows that $u^{-1}(L)$ can only accumulate at $\operatorname{Crit}(u) \cup \partial D$. On the other hand, since $L$ is totally real, any non-critical point of $u$ in $\partial D$ is a point where the intersection of $u$ with $L$ is clean. These points can therefore not be accumulated by points of $u^{-1}(L) \cap$ int $D \backslash\{0\}$. This completes the proof of (iii).

In the next lemma, $D^{+}:=D \cap\{\operatorname{Im} z \geq 0\}$. Recall that $\alpha$ is the one-form obtained by $\alpha:=\lambda_{\mid M}$ on $M$ and is extended by invariance under the $\mathbb{R}_{*}^{+}$-action on $T^{*} L \backslash L$. It is not defined on $L$.

Lemma 3.8. Let $u:\left(D^{+},[-1,1]\right) \rightarrow\left(T^{*} L, L\right)$ be a $J_{g}$-holomorphic map. Assume that $\varepsilon_{n} \rightarrow 0$ verifies $u\left(D^{+} \cap\left\{\operatorname{Im} z=\varepsilon_{n}\right\}\right) \cap L=\emptyset$. Then,

$$
\lim _{n \rightarrow \infty} \int_{D^{+} \cap\left\{\operatorname{Im} z=\varepsilon_{n}\right\}} u^{*} \alpha=-\ell_{g}\left(u_{\mid[-1,1]}\right),
$$


where $D^{+} \cap\left\{\operatorname{Im} z=\varepsilon_{n}\right\}$ is oriented from left to right.

Notice that both sides of equality (7) are well-defined. The assumption guarantees that $u^{*} \alpha$ is well-defined on $D^{+} \cap\left\{\operatorname{Im} z=\varepsilon_{n}\right\}$. Moreover, by ellipticity of $\bar{\partial}_{J_{g}}, u$ is smooth up to $[-1,1]$, so $u([-1,1])$ has finite length.

Proof: Since both sides of the equality (7) are additive with respect to a partition of Op $\left([-1,1], D^{+}\right)$, we can freely assume that $u$ takes values in a small chart of $T^{*} L$ localized on $L$, which amounts to assuming that $L=\mathbb{R}^{n}$. Let $\gamma:=u_{\mid[-1,1]}$ and $\gamma_{\varepsilon}:=u_{\mid D^{+} \cap\{\operatorname{Im} z=\varepsilon\}}$. Taking into account that for the coordinates $x+i y \in D^{+}$,

$$
\partial_{y} u(t, 0)=J_{g} \partial_{x} u(t, 0)=J_{g} \dot{\gamma}(t)=-g_{\gamma(t)}(\dot{\gamma}(t), \cdot) \in T_{\gamma(t)}^{*} \mathbb{R}^{n}
$$

we have

$$
\begin{aligned}
& \dot{\gamma}_{\varepsilon}(t)=\dot{\gamma}(t)+O(\varepsilon), \\
& \gamma_{\varepsilon}(t)=\left(\gamma(t),-\varepsilon g_{\gamma(t)}(\dot{\gamma}(t), \cdot)\right)+O\left(\varepsilon^{2}\right) .
\end{aligned}
$$

Thus $p\left(\gamma_{\varepsilon}(t)\right):=p_{\gamma_{\varepsilon}(t)}=-\varepsilon g_{\gamma(t)}(\dot{\gamma}(t), \cdot)+O\left(\varepsilon^{2}\right)$ and $\left\|p\left(\gamma_{\varepsilon}(t)\right)\right\|_{g}=\varepsilon\|\dot{\gamma}(t)\|_{g}+O\left(\varepsilon^{2}\right)$. If $\dot{\gamma}(t) \neq 0$, this implies

$$
\alpha\left(\gamma_{\varepsilon}(t)\right):=\alpha_{\gamma_{\varepsilon}(t)}=\frac{p}{\|p\|_{g}}\left(\gamma_{\varepsilon}(t)\right)=-g_{\gamma(t)}\left(\frac{\dot{\gamma}(t)}{\|\dot{\gamma}(t)\|_{g}}, \cdot\right)+O(\varepsilon) .
$$

Notice now that, using the reflection argument as in lemma 3.7, one immediately sees that the critical set of the restriction of $u$ to any compact set of $(-1,1)$ is a finite set, so the Lebesgue measure $\mathcal{L} e b(\operatorname{Crit}(\gamma))=0$. Thus, one can cover $\operatorname{Crit}(\gamma)$ by an open set $J_{\delta} \subset(-1,1)$ of total length $\delta$. We can also assume that $J_{\delta}$ contains some neighbourhood of $\{-1,1\}$, so that for some $\varepsilon(\delta)>0, \gamma_{\varepsilon}(t)$ is well-defined for $t \in(-1,1) \backslash J_{\delta}$ for all $\varepsilon<\varepsilon(\delta)$. Then, for $\varepsilon_{n}<\varepsilon(\delta)$, we have

$$
\begin{aligned}
\int_{(-1,1) \backslash J_{\delta}+i \varepsilon_{n}} u^{*} \alpha & =\int_{(-1,1) \backslash J_{\delta}} \alpha_{\gamma_{\varepsilon_{n}}(t)}\left(\dot{\gamma}_{\varepsilon_{n}}(t)\right) d t \\
& =-\int_{(-1,1) \backslash J_{\delta}} g_{\gamma(t)}\left(\frac{\dot{\gamma}(t)}{\|\dot{\gamma}(t)\|_{g}}, \dot{\gamma}(t)+O\left(\varepsilon_{n}\right)\right)+O\left(\varepsilon_{n}\right) d t \\
& =-\int_{(1,-1) \backslash J_{\delta}}\|\dot{\gamma}(t)\|_{g}+O\left(\varepsilon_{n}\right) d t .
\end{aligned}
$$

Notice also that our $O\left(\varepsilon_{n}\right)$ depends on $u$ and $\delta$, but is uniform in $t$, so

$$
\lim _{n \rightarrow \infty} \int_{(-1,1) \backslash J_{\delta}+i \varepsilon_{n}} u^{*} \alpha=-\ell_{g}\left(\gamma\left((-1,1) \backslash J_{\delta}\right)\right) .
$$

Letting now $\delta$ go to 0 , the right hand side of this equality obviously converges to $-\ell_{g}(\gamma)$ because $\gamma$ is smooth. On the other hand,

$$
\left|\int_{J_{\delta}+i \varepsilon_{n}} u^{*} \alpha\right| \leq \int_{J_{\delta}}\left|\frac{p}{\|p\|_{g}}\right|_{\gamma_{\varepsilon_{n}}(t)}\left(\dot{\gamma}_{\varepsilon_{n}}(t)\right) \mid d t \leq \int_{J_{\delta}}\left\|\dot{\gamma}_{\varepsilon_{n}}(t)\right\|_{g} d t \leq C \delta,
$$

where $C$ is an upper bound for $\|d u\|_{g}$. This shows equality (7). 
Lemma 3.9. If $\gamma$ is a geodesic of minimal length in its homology class $\beta$, then $u_{\gamma, g}$ is the only $J_{g}$-holomorphic punctured disc with boundary on $L$ and asymptotic to $\tilde{\gamma}$ at 0 up to $S^{1}$-reparametrization. In other terms,

$$
\mathcal{M}\left(J_{g}, \beta\right)=\left\{\left[u_{\gamma, g}\right]\right\} .
$$

Proof: Let $u \in \widehat{\mathcal{M}}\left(J_{g}, \beta\right)$. By lemma 3.7 we have $u^{-1}(L)=\mathcal{I} \cup \partial D$, where $\mathcal{I} \subset$ int $D \backslash\{0\}$ can only accumulate at critical points of $u$. On $\Omega:=D \backslash\left(u^{-1}(L) \cup\{0\}\right)$ we can write $u=(a, \tilde{u}) \in(0, \infty) \times M$. Calling $P: T M \rightarrow \operatorname{ker} \alpha$ the projection along the Reeb vector field and putting $V=\langle V, R\rangle R+P(V)$ for $V \in T M$, the equations for a $J_{g}$-holomorphic map can be written

$$
\begin{aligned}
& d a=\chi(a)\langle d \tilde{u} \circ j, R\rangle, \\
& J_{g}(u) P(d \tilde{u})=P(d \tilde{u} \circ j) .
\end{aligned}
$$

As a result, if $s+i t$ are local holomorphic coordinates near some point in $\Omega$,

$u^{*} d \alpha\left(\frac{\partial}{\partial s}, \frac{\partial}{\partial t}\right)=\tilde{u}^{*} d \alpha\left(\frac{\partial}{\partial s}, \frac{\partial}{\partial t}\right)=d \alpha\left(\frac{\partial \tilde{u}}{\partial s}, \frac{\partial \tilde{u}}{\partial t}\right)=d \alpha\left(P \frac{\partial \tilde{u}}{\partial s}, P \frac{\partial \tilde{u}}{\partial t}\right)=d \alpha\left(P \frac{\partial \tilde{u}}{\partial s}, J_{g} P \frac{\partial \tilde{u}}{\partial s}\right) \geqslant 0$,

and equality holds if and only if $P \frac{\partial \tilde{u}}{\partial s}=P \frac{\partial \tilde{u}}{\partial t}=0$.

Fix now $\varepsilon \ll 1$ such that $\partial D(0,1-\varepsilon)$ avoids $\mathcal{I}$. Since $\mathcal{I}$ can only accumulate at critical points of $u$ and $\operatorname{Crit}(u)$ is finite, it is possible to find a finite number of disjoint disks $D\left(\zeta_{j}, \varepsilon_{j}\right) \Subset D(0,1-\varepsilon)$ such that $\mathcal{I} \cap D(0,1-\varepsilon) \subset \bigcup_{j} D\left(\zeta_{j}, \varepsilon_{j}\right)$. Here the $\varepsilon_{j}$ are chosen small enough and the number of disjoint disks $k$ depends on these choices. Let also $\gamma_{\varepsilon}^{j}:=\partial D\left(\zeta_{j}, \varepsilon_{j}\right), \gamma_{\varepsilon}:=\partial D(0, \varepsilon), \gamma_{1-\varepsilon}:=\partial D(0,1-\varepsilon)$, all those circles being oriented as the boundary of $\partial \Omega_{\varepsilon}:=\partial\left(D(0,1-\varepsilon) \backslash\left(D(0, \varepsilon) \cup \bigcup_{j} D\left(\zeta_{j}, \varepsilon_{j}\right)\right)\right)$. Then,

$$
0 \leq \int_{\Omega_{\varepsilon}} u^{*} d \alpha=\int_{\partial \Omega_{\varepsilon}} u^{*} \alpha=\sum_{j=1}^{k} \int_{\gamma_{\varepsilon}^{j}} u^{*} \alpha+\int_{\gamma_{\varepsilon}} u^{*} \alpha+\int_{\gamma_{1-\varepsilon}} u^{*} \alpha .
$$

Since $u$ is asymptotic to $\tilde{\gamma}$ at 0 , a straightforward computation shows that

$$
\lim _{\varepsilon \rightarrow 0} \int_{\gamma_{\varepsilon}} u^{*} \alpha=\ell_{g}(\gamma)
$$

On the other hand, since $\alpha=p /\|p\|_{g}$ is a bounded 1-form on $T^{*} L \backslash L$, its integral over the small loops $u \circ \gamma_{\varepsilon}^{j}$ tend to 0 when $\varepsilon_{j} \rightarrow 0$ (recall that $u$ is smooth near the $\zeta_{j}$ ). Since $u$ is smooth on int $D \backslash\{0\}$ and $\mathcal{I}$ does not approach 0 , we can assume that

$$
\left|\sum_{j=1}^{k} \int_{\gamma_{\varepsilon}^{j}} u^{*} \alpha\right|<\varepsilon
$$

by decreasing $\varepsilon_{j}$ and increasing $k$ if necessary. Finally, taking orientation into account, lemma 3.8 shows that

$$
\lim _{\varepsilon \rightarrow 0} \int_{\gamma_{1-\varepsilon}} u^{*} \alpha=-\ell_{g}(u(\partial D)) .
$$


Putting all these estimates together we get

$$
0 \leq \int_{\Omega} u^{*} d \alpha=\ell_{g}(\gamma)-\ell_{g}(u(\partial D))
$$

However the reverse inequality holds as well, because the projection of $u$ to $L$ provides a singular chain between $\gamma$ and $u(\partial D)$ and $\gamma$ is the geodesic of minimal length in its homology class. We therefore conclude that $u^{*} d \alpha=\tilde{u}^{*} d \alpha=0$ on $\Omega=D \backslash(\mathcal{I} \cup\{0\})$, so $\pi \circ d \tilde{u}=0$ on $\Omega$, which means that $\operatorname{Im} d u(z) \in\left\langle R, \frac{\partial}{\partial r}\right\rangle \forall z \in \Omega$. Since $u$ is asymptotic to $\tilde{\gamma}$ at 0 , we see that $\operatorname{Im} u=\operatorname{Im} u_{\gamma, g}$. But then $u=u_{\gamma, g}$ modulo source reparametrization.

\subsection{Transversality}

The object of this paragraph is to study the surjectivity of the linearized Cauchy-Riemann operator at $u_{\gamma, g}$. We recall that

- $(L, g)$ determines $M=\{g=1\}, \alpha, R$,

- $T_{(q, p)}\left(T^{*} L\right)=\left\langle\frac{\partial}{\partial r}, R(q, p)\right\rangle \oplus \operatorname{ker} \alpha \cap F_{(q, p)} \oplus \operatorname{ker} \alpha \cap H_{(q, p)} \forall q \in L, p \neq 0$,

- $J_{g} R=-\chi(r) g_{q}(\pi(R), \cdot)=-\chi(r) \frac{\partial}{\partial r}$, where $\chi(r)=1$ near $r=0$ and $\chi(r)=r$ near $\infty$,

- $J_{g} V=-g_{q}(\pi(V), \cdot) \in \operatorname{ker} \alpha \cap F_{(q, p)}$ for $V \in \operatorname{ker} \alpha \cap H_{(q, p)}$,

- $\gamma$ is a minimizing geodesic in the class $\beta$ whose length $\ell_{g}(\gamma)$ will be denoted $\ell$,

- $u_{\gamma, g}(s, t)=(f(s), \tilde{\gamma}(t)) \in(0, \infty) \times M \subset T^{*} L$ and $f(s):=G^{-1}(s)$ with $G(r)=\int_{0}^{r} \frac{d r}{\chi(r)}$. In fact $f$ solves the differential equation $f^{\prime}=\chi \circ f$.

The functional analytic setup is as follows. Since this approach is fairly standard, we refer the reader to the appendix (page 61) for the precise definitions and recall the main points here. We define a Banach manifold of maps $\mathcal{B}^{k, p, \delta}$ that contains $u_{\gamma, g}$. We consider a Banach space bundle $\mathcal{E}^{k-1, p, \delta} \rightarrow \mathcal{B}^{k, p, \delta}$ whose fibers are spaces of complex antilinear bundle maps. The non-linear Cauchy Riemann operator $\bar{\partial}_{J_{g}}$ defines a smooth section of this bundle by

$$
\bar{\partial}_{J_{g}}(u)=d u+J_{g}(u) \circ d u \circ j
$$

and we have $\bar{\partial}_{J_{g}}\left(u_{\gamma, g}\right)=0$. The linearization of $\bar{\partial}_{J_{g}}$ at $u_{\gamma, g}$ is given by

$$
\begin{aligned}
\mathbf{D}_{u_{\gamma, g}}: T_{u_{\gamma, g}} \mathcal{B}^{k, p, \delta} & \longrightarrow \mathcal{E}_{u_{\gamma, g}-1, p, \delta} \\
\xi & \longmapsto \nabla \xi+J_{g} \circ \nabla \xi \circ j+\left(\nabla_{\xi} J_{g}\right) \circ d u_{\gamma, g} \circ j,
\end{aligned}
$$

where $\nabla$ is any symmetric connection on $T^{*} L$. Using local coordinates in a neighborhood of our geodesic our operator takes the form

$$
\mathbf{D}_{u_{\gamma, g}}(\xi)=\bar{\partial}_{J_{g}} \xi+\left(d J_{g}(\xi)\right) d u_{\gamma, g} \circ j:=d \xi+J_{g}(u) \circ d \xi \circ j+\left(d J_{g}(\xi)\right) d u_{\gamma, g} \circ j .
$$


In the appendix we show that this operator is Fredholm and has index 1 if $\gamma$ is minimal (see corollary A.7). By elliptic regularity the kernel of $\mathbf{D}_{u_{\gamma, g}}$, which will be our only concern, does not depend on $k, p$ and $\delta$ provided $k p>2$ and $\delta>0$ small enough.

The idea is clear: the linearized operator $\mathbf{D}_{u_{\gamma, g}}$ is Fredholm and has index 1. Establishing surjectivity therefore amounts to proving that its kernel has dimension 1. Introducing appropriate coordinates we manage to compute the operator explicitly. Under certain conditions on the metric we then compute its kernel and show that it has the right dimension. In the next section we will show that the metrics that satisfy these conditions are $\mathcal{C}^{0}$-dense in the set of Riemannian metrics on $L$.

We first need to introduce good coordinates, called Fermi coordinates, in a neighbourhood of our closed geodesic $\gamma$. Unfortunately, without any assumption on the parallel transport along $\gamma$, these coordinates are multivalued (equivalently, they only give local coordinates on some cover of $L$ ), so we need to introduce a new set of notation. Let $\left(\dot{\gamma}(0), v_{1}, \ldots, v_{n-1}\right)$ be an orthonormal basis of $T_{\gamma(0)} L$, and let $\left(\dot{\gamma}(t), V_{1}(t), \ldots, V_{n-1}(t)\right)$ be the orthonormal basis obtained by parallel transport of $\left(\dot{\gamma}(0), v_{1}, \ldots, v_{n-1}\right)$ along $\gamma_{\mid[0, t]}$. We define

$$
\begin{aligned}
\varphi: \mathbb{R} \times(-1,1)^{n-1} & \longrightarrow L \\
\left(x_{n}, x^{\prime}\right) & \longmapsto \exp _{\gamma\left(x_{n}\right)}\left(\sum_{i=1}^{n-1} x_{i} V_{i}\left(x_{n}\right)\right) \quad\left(x^{\prime}=\left(x_{1}, \ldots, x_{n-1}\right)\right) .
\end{aligned}
$$

By restricting this map to $\mathbb{R} \times(-\delta, \delta)^{n-1}$ for $\delta>0$ small enough we get an immersion. This can alternatively be achieved on $\mathbb{R} \times(-1,1)^{n-1}$ by rescaling the metric. Such a rescaling obviously does not affect the generality of our argument, so we assume that $\varphi$ itself is an immersion. When the parallel transport $O$ along $\gamma_{\mid[0, \ell]}$ is the identity, $\varphi$ induces an embedding $S^{1} \times(-1,1)^{n-1} \hookrightarrow L$, which provides coordinates near $\gamma$. In general however, this immersion only provides multivalued coordinates (it satisfies $\varphi\left(x_{n}+\ell, x^{\prime}\right)=\varphi\left(x_{n}, O x^{\prime}\right)$ ). The map $\varphi$ naturally lifts to an immersion

$$
\Phi: T^{*}\left(\mathbb{R} \times(-1,1)^{n-1}\right) \leftrightarrow T^{*} L
$$

such that $\Phi\left(x_{n}+\ell, y_{n}, x^{\prime}, y^{\prime}\right)=\Phi\left(x_{n}, y_{n}, O x^{\prime},{ }^{t} O^{-1} y^{\prime}\right)=\Phi\left(x_{n}, y_{n}, O x^{\prime}, O y^{\prime}\right)$. Here $\left(y_{n}, y^{\prime}\right) \in$ $\mathbb{R}^{n}$ are the coordinates in the fiber. We define $\hat{g}, \hat{J}_{g}, \hat{\alpha}, \ldots$ to be the pull-backs of their corresponding objects in $T^{*} L$ by $\Phi$. By construction, $\hat{g}_{i j}=\delta_{i j}+O\left(\left\|x^{\prime}\right\|^{2}\right)$, where $\|\cdot\|$ is any norm on $\mathbb{R}^{n-1}$ (equivalently, $\hat{g}_{i j}\left(x_{n}, 0\right)=\delta_{i j}$ and $\frac{\partial \hat{g}_{i j}}{\partial x_{l}}\left(x_{n}, 0\right)=0$ ). Notice that by functoriality of the Liouville form, we get $\hat{\alpha}=\alpha_{\hat{g}}$, so $\hat{R}=R_{\hat{g}}, \ldots$, and finally $\hat{J}_{g}=J_{\hat{g}}$ (provided we use $\hat{\chi}=\Phi^{*} \chi$ in the definition of $J_{\hat{g}}$ ). Also, $\gamma$ pulls-back to $\hat{\gamma}(t)=(t, 0) \in$ $\mathbb{R} \times(-1,1)^{n-1}, \tilde{\gamma}$ to $\tilde{\hat{\gamma}}$, and $u_{\gamma, g}$ to the map

$$
\begin{aligned}
\hat{u}_{\gamma, g}=u_{\hat{\gamma}, \hat{g}}:[0, \infty) \times \mathbb{R} & \longrightarrow T^{*}\left(\mathbb{R} \times(-1,1)^{n-1}\right) \simeq \mathbb{R}_{\left(x_{n}, y_{n}\right)}^{2} \times(-1,1)_{x^{\prime}}^{n-1} \times \mathbb{R}_{y^{\prime}}^{n-1} \\
(s, t) & \longmapsto(t, f(s), 0,0)
\end{aligned}
$$

This map is $J_{\hat{g}}$-holomorphic for the standard holomorphic structure $j \partial_{s}=\partial_{t}$ on $[0, \infty) \times \mathbb{R}$ (recall that $J_{\hat{g}}\left(x_{n}, y_{n}, 0,0\right) \frac{\partial}{\partial x_{n}}=-\chi\left(y_{n}\right) \frac{\partial}{\partial y_{n}}$ ). Finally, $\xi$ lifts to a vector field $\hat{\xi}$ along $u_{\hat{\gamma}, \hat{g}}$

$$
\begin{aligned}
\hat{\xi}:[0, \infty) \times \mathbb{R} & \longrightarrow T_{\hat{u}_{\gamma, g}}\left(T^{*}\left(\mathbb{R} \times(-1,1)^{n-1}\right)\right) \simeq \mathbb{R}^{2} \times \mathbb{R}^{2 n} \\
(s, t) & \longmapsto(t, f(s), \hat{z}(s, t))
\end{aligned}
$$


(the tangent bundle to $T^{*}\left(\mathbb{R} \times(-1,1)^{n-1}\right)$ is trivial). The vector space $\mathbb{R}^{2 n}$ naturally splits into $\mathbb{R}^{2} \times \mathbb{R}^{2(n-1)}$ tangent to $T^{*} \mathbb{R}$ and to $T^{*}(-1,1)^{n-1}$, respectively. Both factors $\mathbb{R}^{2}$ and $\mathbb{R}^{2(n-1)}$ further split as $\mathbb{R} \times \mathbb{R}$ and $\mathbb{R}^{n-1} \times \mathbb{R}^{n-1}$, where the second factors are tangents to the fibers of the cotangent bundles, while the first factors are non-canonical "horizontal spaces" (not to be confused with the subbundle $H$ ), which are tangent to $L$ at the zero-section. We thus write

$$
\begin{aligned}
\hat{z}(s, t) & =\left(\hat{z}_{n}(s, t), \hat{z}^{\prime}(s, t)\right) \\
& =\left(\hat{a}_{n}(s, t), \hat{b}_{n}(s, t), \hat{a}^{\prime}(s, t), \hat{b}^{\prime}(s, t)\right) \in \mathbb{R}^{2} \times \mathbb{R}^{2(n-1)} \\
& =\left(\hat{a}_{n}(s, t), \hat{b}_{n}(s, t), \hat{a}_{1}(s, t), \ldots, \hat{a}_{n-1}(s, t), \hat{b}_{1}(s, t), \ldots, \hat{b}_{n-1}(s, t)\right) .
\end{aligned}
$$

Then, $\hat{z}(s, t)$ has the following properties (see appendix A.2):

1. $\hat{z} \in W_{\mathrm{loc}}^{k, p}\left([0, \infty) \times \mathbb{R}, \mathbb{R}^{2 n}\right)$,

2. $\hat{z}(0, t)$ is tangent to the zero section $\left(\right.$ i.e. $\left.\hat{b}_{i}(0, t)=0 \forall i\right)$

3. $\left\|\hat{z}^{\prime}(s, t)\right\| \leq C e^{-\delta s}$ for some constant $C$. In particular, $\hat{a}^{\prime}$ and $\hat{b}^{\prime}$ tend to 0 uniformly when $s$ tends to $+\infty$,

4. $\hat{a}_{n}(s, \cdot)$ and $\hat{b}_{n}(s, \cdot)$ tend to constants uniformly when $s$ tends to $+\infty$

5. Finally, $\hat{z}$ satisfies a certain pseudo-periodicity with regard to the parallel transport map $O$. Recall that $\Phi\left(x_{n}+\ell, y_{n}, x^{\prime}, y^{\prime}\right)=\Phi\left(x_{n}, y_{n}, O x^{\prime}, O y^{\prime}\right)$, thus we have

$$
\hat{z}_{n}(s, t+\ell)=\hat{z}_{n}(s, t), \quad \hat{z}^{\prime}(s, t+\ell)=O \hat{z}^{\prime}(s, t):=\left(O \hat{a}^{\prime}(s, t), O \hat{b}^{\prime}(s, t)\right) .
$$

We call $W$ the space of maps $\hat{z}:[0, \infty) \times \mathbb{R} \rightarrow \mathbb{R}^{2 n}$ that satisfy the four properties listed above. For $\hat{z} \in W$ and $\hat{\xi}=(t, f(s), \hat{z}(s, t))$, we then define

$$
\hat{D} \hat{\xi}:=\bar{\partial}_{\hat{J}_{g}} \hat{\xi}+\left(d \hat{J}_{g}(\hat{\xi})\right) d \hat{u}_{\gamma, g} \circ j .
$$

The naturality of the lift $\Phi$ of $\varphi$ readily implies that for $\Phi_{*} \hat{\xi}=\xi$

$$
\Phi_{*} \hat{D} \hat{\xi}=\mathbf{D}_{u_{\gamma, g}}(\xi),
$$

so if $\xi$ lies in the kernel of $\mathbf{D}_{u_{\gamma, g}}, \hat{D} \hat{\xi}=0$ as well. Thus, $\mathbf{D}_{u_{\gamma, g}}$ is surjective as soon as $\operatorname{dim} \operatorname{ker} \hat{D}=1$. We can get a more explicit expression for $\hat{D}$ by noticing that

- $\hat{D} \hat{\xi}$ is a $(0,1)$-operator, so it is determined by its action on $\frac{\partial}{\partial s}$. We will therefore identify $\hat{D} \hat{\xi}$ with $\hat{D} \hat{\xi}\left(\frac{\partial}{\partial s}\right)$. Moreover, $d u_{\hat{\gamma}, \hat{g}} \circ j \frac{\partial}{\partial s}=\frac{\partial}{\partial x_{n}}$

- $J_{\hat{g}}$ is a tensor field on $T^{*}\left(\mathbb{R} \times(-1,1)^{n-1}\right) \simeq \mathbb{R}^{2} \times(-1,1)^{n-1} \times \mathbb{R}^{n-1}$, whose tangent bundle is trivial. It can therefore be seen as a map $J_{\hat{g}}: \mathbb{R}^{2} \times(-1,1)^{n-1} \times \mathbb{R}^{n-1} \rightarrow$ $M_{2 n}(\mathbb{R})$. Via this identification, $d J_{\hat{g}}(\hat{\xi})=d J_{\hat{g}}(\hat{z})$ and $\bar{\partial}_{\hat{g}} \hat{\xi} \frac{\partial}{\partial s}=\frac{\partial \hat{z}}{\partial s}+J_{\hat{g}} \frac{\partial \hat{z}}{\partial t}$. 
Thus, putting $\hat{J}(s, t):=J_{\hat{g}}\left(u_{\hat{\gamma}, \hat{g}}(s, t)\right)=J_{\hat{g}}(t, f(s), 0,0)$, we get

$$
\begin{aligned}
\hat{D} \hat{\xi}\left(\frac{\partial}{\partial s}\right) & =\frac{\partial \hat{z}}{\partial s}+\hat{J}(s, t) \frac{\partial \hat{z}}{\partial t}+d J_{\hat{g}}(\hat{z}) \frac{\partial}{\partial x_{n}} \\
& =\frac{\partial z}{\partial s}+\hat{J}(s, t) \frac{\partial \tilde{z}}{\partial t}+d\left(J_{\hat{g}} \frac{\partial}{\partial x_{n}}\right) \hat{z} .
\end{aligned}
$$

Let us also finally simplify our notation. In the remainder of this section no further reference to $\hat{g}, \hat{J}_{g}, \hat{u}_{\gamma, g}, \hat{\xi}$ will be made. We only pay attention to our operator $\hat{D}$ that lives on the pull-back space. In order to keep the notation as light as possible, we therefore suppress all hats ${ }^{\wedge}$ from our letters, remembering that all objects correspond to their pull-backs by $\Phi$. In other terms, in the rest of this section, $\left.\gamma(t)=(t, 0) \in \mathbb{R} \times(-1,1)^{n-1}, u_{\gamma, g}=(t, f(s), 0,0)\right) \in$ $T^{*} \mathbb{R} \times T^{*}(-1,1)^{n-1}, g=\Phi^{*} g, J_{g}=J_{\hat{g}} \ldots$ We only call $\hat{L}:=\mathbb{R} \times(-1,1)^{n}$ in order to keep the reader aware that we work in our cover.

Summarizing our discussion we obtain the following.

Proposition 3.10. Our initial linearized Cauchy-Riemann operator is surjective as soon as the kernel of the operator

$$
\begin{aligned}
D: W & \longrightarrow W^{k-1, p}\left([0, \infty) \times \mathbb{R}, \mathbb{R}^{2 n}\right) \\
z & \longmapsto \frac{\partial z}{\partial s}+J_{g}(s, t) \frac{\partial z}{\partial t}+d\left(J_{g} \frac{\partial}{\partial x_{n}}\right) z
\end{aligned}
$$

has dimension 1.

We now wish to explicitly compute our operator $D$ in the coordinates $\left(x_{n}, y_{n}, x^{\prime}, y^{\prime}\right)$ that we have introduced in the neighbourhood of $\operatorname{Im} u_{\gamma, g}$. Recall that $g$ is flat of order 1 near $\gamma$ in these coordinates $\left(g_{i j}=\delta_{i j}+O\left(\left\|x^{\prime}\right\|^{2}\right)\right)$.

Lemma 3.11. On $T^{*} \hat{L}$, endowed with the coordinates $\left(x_{n}, y_{n}, x_{1}, \ldots, x_{n-1}, y_{1}, \ldots, y_{n-1}\right)$ defined above, we have at $\left(x_{n}, y_{n}, 0,0\right), y_{n} \geq 0$,

- $J_{g} \frac{\partial}{\partial x_{i}}=-\frac{\partial}{\partial y_{i}} \forall i<n \quad$ and $\quad J_{g} \frac{\partial}{\partial x_{n}}=-\chi\left(y_{n}\right) \frac{\partial}{\partial y_{n}}$

- $\frac{\partial J_{g}}{\partial y_{i}}\left(\frac{\partial}{\partial x_{n}}\right)=\frac{1-\chi\left(y_{n}\right)}{y_{n}} \frac{\partial}{\partial y_{i}} \forall i<n \quad$ and $\quad \frac{\partial J_{g}}{\partial y_{n}}\left(\frac{\partial}{\partial x_{n}}\right)=-\chi^{\prime}\left(y_{n}\right) \frac{\partial}{\partial y_{n}}$,

- $\frac{\partial}{\partial x_{i}}\left(J_{g} \frac{\partial}{\partial x_{n}}\right)=-\frac{y_{n}}{2} \sum_{l \neq n} \frac{\partial^{2} g_{n n}}{\partial x_{i} \partial x_{l}} \frac{\partial}{\partial x_{l}} \forall i<n \quad$ and $\quad \frac{\partial}{\partial x_{n}}\left(J_{g} \frac{\partial}{\partial x_{n}}\right)=0$.

Proof: Until now, we have not made a distinction between the metric $g$ on the manifold and the induced metric on the cotangent bundle. In this proof alone, we need to make a clear distinction, so the metric in the cotangent bundle will be denoted $g^{\sharp}$. By definition of the Fermi coordinates the vector fields $\left(\partial / \partial x_{i}\right)$ are parallel along the geodesic $\gamma$, so the vectors $\left(\partial / \partial x_{i}\right)$ belong to the horizontal space at every point of the lift of $\gamma$, which are precisely the points $\left(x_{n}, y_{n}, 0,0\right)$. Since $\alpha=\operatorname{sign}\left(y_{n}\right) d x_{n}$ at these points, we thus see that $\frac{\partial}{\partial x_{i}} \in \operatorname{ker} \alpha \cap H$ for $i \neq n$, so

$$
J_{g} \frac{\partial}{\partial x_{i}}\left(x_{n}, y_{n}, 0,0\right)=-\frac{\partial}{\partial y_{i}} \quad \forall i \neq n
$$


Since $R$ flows along the cogeodesic flow in such a way that its projection on $L$ has speed 1 , it is also clear that $R\left(x_{n}, y_{n}, 0,0\right)=\partial / \partial x_{n}$, so

$$
J_{g} \frac{\partial}{\partial x_{n}}\left(x_{n}, y_{n}, 0,0\right)=-\chi\left(y_{n}\right) \frac{\partial}{\partial y_{n}} .
$$

This proves the first assertion of the lemma and shows as well

$$
\frac{\partial}{\partial x_{n}}\left(J_{g} \frac{\partial}{\partial x_{n}}\right)\left(x_{n}, y_{n}, 0,0\right)=0, \quad \frac{\partial}{\partial y_{n}}\left(J_{g} \frac{\partial}{\partial x_{n}}\right)\left(x_{n}, y_{n}, 0,0\right)=-\chi^{\prime}\left(y_{n}\right) \frac{\partial}{\partial y_{n}} .
$$

In order to compute the other derivatives, we need to compute $J_{g} \frac{\partial}{\partial x_{n}}\left(x_{n}, y_{n}, x^{\prime}, y^{\prime}\right)$ at the first order, which requires to decompose $\partial / \partial x_{n}$ along $\left\langle R, \frac{\partial}{\partial r}\right\rangle \oplus \operatorname{ker} \alpha \cap H \oplus \operatorname{ker} \alpha \cap F$.

Preliminary step: computation of $R . R$ is colinear to the Hamiltonian vector field associated to the function $H(x, y):=\|y\|_{g_{x}^{\sharp}}^{2}=\sum g_{i j}^{\sharp}(x) y_{i} y_{j}$, because both generate the cogeodesic flow on the level sets of $H$, which coincide with the hypersurfaces $\{r\} \times M$. This shows that for $r>0, R$ is colinear to

$$
\sum_{l}\left(\left(\sum_{i, j} \frac{\partial g_{i j}^{\sharp}}{\partial x_{l}} y_{i} y_{j}\right) \frac{\partial}{\partial y_{l}}-2\left(\sum_{j} g_{l j}^{\sharp} y_{j}\right) \frac{\partial}{\partial x_{l}}\right) .
$$

We specialize to two situations. When $x^{\prime}=0$, we have $\frac{\partial g_{i j}^{\sharp}}{\partial x_{l}}=0$ and $g_{l j}^{\sharp}=\delta_{l j}$, so for $y_{n}>0$

$$
R\left(x_{n}, y_{n}, 0, y^{\prime}\right) \propto R^{\prime}:=\frac{\partial}{\partial x_{n}}+\sum_{l \neq n} \frac{y_{l}}{y_{n}} \frac{\partial}{\partial x_{l}} .
$$

Taking again into account that $g_{i j}\left(x_{n}, 0\right)=\delta_{i j}$ and that $\pi(R)^{\sharp}=\frac{\partial}{\partial r}$, we thus get

$$
\frac{\partial}{\partial r}\left(x_{n}, y_{n}, 0, y^{\prime}\right) \propto \pi\left(R^{\prime}\right)^{\sharp}=g\left(\pi\left(R^{\prime}\right), \cdot\right)=\frac{\partial}{\partial y_{n}}+\sum_{l \neq n} \frac{y_{l}}{y_{n}} \frac{\partial}{\partial y_{l}} .
$$

When $y^{\prime}=0$, we get

$$
R\left(x_{n}, y_{n}, x^{\prime}, 0\right) \propto \sum_{l} \frac{\partial g_{n n}^{\sharp}}{\partial x_{l}} y_{n}^{2} \frac{\partial}{\partial y_{l}}-2 \sum_{l} g_{l n}^{\sharp} y_{n} \frac{\partial}{\partial x_{l}}
$$

Taking into account that $g_{l n}^{\sharp}\left(x_{n}, x^{\prime}\right)=\delta_{l n}+O\left(\left\|x^{\prime}\right\|^{2}\right)$, we therefore get

$$
R\left(x_{n}, y_{n}, x^{\prime}, 0\right) \propto R^{\prime}=\frac{\partial}{\partial x_{n}}-\frac{y_{n}}{2} \sum_{l} \frac{\partial g_{n n}^{\sharp}}{\partial x_{l}} \frac{\partial}{\partial y_{l}}+O\left(\left\|x^{\prime}\right\|^{2}\right),
$$

and

$$
\frac{\partial}{\partial r}\left(x_{n}, y_{n}, x^{\prime}, 0\right) \propto \pi\left(R^{\prime}\right)^{\sharp}=g\left(\frac{\partial}{\partial x_{n}}, \cdot\right)+O\left(\left\|x^{\prime}\right\|^{2}\right)=\frac{\partial}{\partial y_{n}}+O\left(\left\|x^{\prime}\right\|^{2}\right) .
$$

Computation of $\frac{\partial}{\partial y_{i}}\left(J_{g} \frac{\partial}{\partial x_{n}}\right)$ for $i<n$. Using the preliminary step we see that

$$
J_{g} \frac{\partial}{\partial x_{n}}\left(x_{n}, y_{n}, 0, y^{\prime}\right)=J_{g}\left(R^{\prime}-\sum_{l \neq n} \frac{y_{l}}{y_{n}} \frac{\partial}{\partial x_{l}}\right) .
$$


Taking into account that $y_{l} \in O\left(\left\|y^{\prime}\right\|\right)$ and $J_{g} \frac{\partial}{\partial x_{l}}=-\frac{\partial}{\partial y_{l}}$ for $l \neq n$ (using equation (5) and the fact that $x^{\prime}=0$ ), we thus get

$$
J_{g} \frac{\partial}{\partial x_{n}}\left(x_{n}, y_{n}, 0, y^{\prime}\right)=-\chi\left(\|y\|_{g^{\sharp}}\right) \pi\left(R^{\prime}\right)^{\sharp}+\sum_{l \neq n} \frac{y_{l}}{y_{n}} \frac{\partial}{\partial y_{l}} .
$$

Since $y_{n}>0, \chi\left(\|y\|_{g^{\sharp}}\right)=\chi\left(y_{n}\right)+O\left(\left\|y^{\prime}\right\|^{2}\right)$, so

$$
J_{g} \frac{\partial}{\partial x_{n}}\left(x_{n}, y_{n}, 0, y^{\prime}\right)=-\chi\left(y_{n}\right) \frac{\partial}{\partial y_{n}}+\sum_{l \neq n} \frac{y_{l}}{y_{n}}\left(1-\chi\left(y_{n}\right)\right) \frac{\partial}{\partial y_{l}}+O\left(\left\|y^{\prime}\right\|^{2}\right) .
$$

Differentiating with respect to $y_{l}$, we get the announced formula for $\frac{\partial}{\partial y_{l}}\left(J_{g} \frac{\partial}{\partial x_{n}}\right)$.

Computation of $\frac{\partial}{\partial x_{i}}\left(J_{g} \frac{\partial}{\partial x_{n}}\right)$ for $i<n$. Using the preliminary step, we can write

$$
\frac{\partial}{\partial x_{n}}\left(x_{n}, y_{n}, x^{\prime}, 0\right)=R^{\prime}+\frac{y_{n}}{2} \sum_{l} \frac{\partial g_{n n}^{\sharp}}{\partial x_{l}} \frac{\partial}{\partial y_{l}}+O\left(\left\|x^{\prime}\right\|^{2}\right) .
$$

Noticing that $\frac{\partial g_{n}^{\sharp}}{\partial x_{l}} \in O\left(\left\|x^{\prime}\right\|\right)$ for all $l, J_{g} \frac{\partial}{\partial y_{l}}=\frac{\partial}{\partial x_{l}}+O\left(\left\|x^{\prime}\right\|\right)$ for all $l \neq n$, and $J_{g} \frac{\partial}{\partial y_{n}}=$ $\frac{1}{\chi\left(y_{n}\right)} \frac{\partial}{\partial x_{n}}+O\left(\left\|x^{\prime}\right\|\right)$, we conclude that

$$
\begin{aligned}
J_{g} \frac{\partial}{\partial x_{n}}\left(x_{n}, y_{n}, x^{\prime}, 0\right) & =-\chi\left(y_{n}\right) \pi\left(R^{\prime}\right)^{\sharp}+\frac{y_{n}}{2 \chi\left(y_{n}\right)} \frac{\partial g_{n n}^{\sharp}}{\partial x_{n}} \frac{\partial}{\partial x_{n}}+\frac{y_{n}}{2} \sum_{l} \frac{\partial g_{n n}^{\sharp}}{\partial x_{l}} \frac{\partial}{\partial x_{l}}+O\left(\left\|x^{\prime}\right\|^{2}\right), \\
& =-\chi\left(y_{n}\right) \frac{\partial}{\partial y_{n}}+\frac{y_{n}}{2 \chi\left(y_{n}\right)} \frac{\partial g_{n n}^{\sharp}}{\partial x_{n}} \frac{\partial}{\partial x_{n}}+\frac{y_{n}}{2} \sum_{l \neq n} \frac{\partial g_{n n}^{\sharp}}{\partial x_{l}} \frac{\partial}{\partial x_{l}}+O\left(\left\|x^{\prime}\right\|^{2}\right) .
\end{aligned}
$$

Since $\sum_{j=1}^{n} g_{n j}^{\sharp}\left(x_{n}, x^{\prime}\right) g_{j n}\left(x_{n}, x^{\prime}\right)=1$ and each factor for $j \neq n$ is $O\left(\left\|x^{\prime}\right\|^{2}\right)$, we see that $g_{n n}^{\sharp}=\frac{1}{g_{n n}}+O\left(\left\|x^{\prime}\right\|^{4}\right)$. Using $g_{n n}\left(x_{n}, x^{\prime}\right)=1+O\left(\left\|x^{\prime}\right\|^{2}\right)$ we obtain $\frac{\partial g_{n n}^{\sharp}}{\partial x_{l}}=-\frac{1}{g_{n n}^{2}} \frac{\partial g_{n n}}{\partial x_{l}}+$ $O\left(\left\|x^{\prime}\right\|^{3}\right)=-\frac{\partial g_{n n}}{\partial x_{l}}+O\left(\left\|x^{\prime}\right\|^{2}\right)$. Differentiating with respect to $x_{j}$ we get

$$
\frac{\partial}{\partial x_{j}}\left(J_{g} \frac{\partial}{\partial x_{n}}\right)\left(x_{n}, y_{n}, 0,0\right)=-\frac{y_{n}}{2 \chi\left(y_{n}\right)} \frac{\partial^{2} g_{n n}}{\partial x_{n} \partial x_{j}} \frac{\partial}{\partial x_{n}}-\frac{y_{n}}{2} \sum_{l \neq n} \frac{\partial^{2} g_{n n}}{\partial x_{j} \partial x_{l}} \frac{\partial}{\partial x_{l}} .
$$

Notice finally that $\frac{\partial g_{n n}}{\partial x_{j}}\left(x_{n}, 0\right) \equiv 0$, so $\frac{\partial^{2} g_{n n}}{\partial x_{n} \partial x_{j}}=0$. We thus get

$$
\frac{\partial}{\partial x_{j}}\left(J_{g} \frac{\partial}{\partial x_{n}}\right)\left(x_{n}, y_{n}, 0,0\right)=-\frac{y_{n}}{2} \sum_{l \neq n} \frac{\partial^{2} g_{n n}}{\partial x_{j} \partial x_{l}} \frac{\partial}{\partial x_{l}},
$$

which is the third point of the lemma.

We are now in position to compute the kernel of our operator $D: W \rightarrow W^{k-1, p}([0, \infty) \times$ $\left.\mathbb{R}, \mathbb{R}^{2 n}\right)$. Recall that $z=\sum a_{i} \frac{\partial}{\partial x_{i}}+b_{i} \frac{\partial}{\partial y_{i}}$ and that

$$
D z=\frac{\partial z}{\partial s}+J_{g}(s, t) \frac{\partial z}{\partial t}+d\left(J_{g} \frac{\partial}{\partial x_{n}}\right) z .
$$


Then,

$$
\begin{aligned}
\frac{\partial z}{\partial s}+J_{g}(s, t) \frac{\partial z}{\partial t}= & \sum_{l} \frac{\partial a_{l}}{\partial s} \frac{\partial}{\partial x_{l}}+\frac{\partial b_{l}}{\partial s} \frac{\partial}{\partial y_{l}}+\sum_{l \neq n}-\frac{\partial a_{l}}{\partial t} \frac{\partial}{\partial y_{l}}+\frac{\partial b_{l}}{\partial t} \frac{\partial}{\partial x_{l}} \\
& -\chi \circ f \frac{\partial a_{n}}{\partial t} \frac{\partial}{\partial y_{n}}+\frac{1}{\chi \circ f} \frac{\partial b_{n}}{\partial t} \frac{\partial}{\partial x_{n}} \\
= & \sum_{l \neq n}\left(\frac{\partial a_{l}}{\partial s}+\frac{\partial b_{l}}{\partial t}\right) \frac{\partial}{\partial x_{l}}+\sum_{l \neq n}\left(\frac{\partial b_{l}}{\partial s}-\frac{\partial a_{l}}{\partial t}\right) \frac{\partial}{\partial y_{l}} \\
& +\left(\frac{\partial a_{n}}{\partial s}+\frac{1}{\chi \circ f} \frac{\partial b_{n}}{\partial t}\right) \frac{\partial}{\partial x_{n}}+\left(\frac{\partial b_{n}}{\partial s}-\chi \circ f \frac{\partial a_{n}}{\partial t}\right) \frac{\partial}{\partial y_{n}}
\end{aligned}
$$

On the other hand,

$$
\begin{aligned}
d\left(J_{g} \frac{\partial}{\partial x_{n}}\right) z= & \sum a_{i} \frac{\partial J_{g}}{\partial x_{i}}\left(\frac{\partial}{\partial x_{n}}\right)+\sum b_{i} \frac{\partial J_{g}}{\partial y_{i}}\left(\frac{\partial}{\partial x_{n}}\right) \\
= & -\sum_{l \neq n}\left(\sum_{i \neq n} a_{i}(s, t) \frac{f(s)}{2} \frac{\partial^{2} g_{n n}}{\partial x_{i} \partial x_{l}}(t)\right) \frac{\partial}{\partial x_{l}} \\
& +\frac{1-\chi \circ f(s)}{f(s)} \sum_{l \neq n} b_{l}(s, t) \frac{\partial}{\partial y_{l}}-b_{n}(s, t) \chi^{\prime} \circ f(s) \frac{\partial}{\partial y_{n}},
\end{aligned}
$$

(we put $\frac{\partial^{2} g_{n n}}{\partial x_{i} \partial x_{j}}(t):=\frac{\partial^{2} g_{n n}}{\partial x_{i} \partial x_{j}}(t, 0)$ ). Taking into account the boundary conditions and the pseudo-periodicity, we finally obtain the following proposition.

Proposition 3.12. $z=\sum a_{i} \frac{\partial}{\partial x_{i}}+b_{i} \frac{\partial}{\partial y_{i}} \in \operatorname{ker} D$ if and only if

$$
\begin{array}{rlrl}
\frac{\partial a_{l}}{\partial s}+\frac{\partial b_{l}}{\partial t}-\frac{f(s)}{2} \sum_{i \neq n} a_{i} \frac{\partial^{2} g_{n n}}{\partial x_{i} \partial x_{l}}(t) & =0 & & \forall l \neq n \\
\frac{\partial b_{l}}{\partial s}-\frac{\partial a_{l}}{\partial t}+\frac{1-\chi \circ f(s)}{f(s)} b_{l} & =0 & & \forall l \neq n \\
\frac{\partial b_{n}}{\partial s}-\chi \circ f(s) \frac{\partial a_{n}}{\partial t}-\chi^{\prime} \circ f(s) b_{n} & =0 & \\
\frac{\partial a_{n}}{\partial s}+\frac{1}{\chi \circ f(s)} \frac{\partial b_{n}}{\partial t} & =0 &
\end{array}
$$

and satisfies the boundary and pseudo-periodicity conditions

$$
\begin{aligned}
& b_{j}(0, t) \equiv 0 \forall j, \quad a_{j}(\infty, t)=b_{j}(\infty, t)=0 \forall j<n, \\
& a_{n}(s, \cdot) \text { and } b_{n}(s, \cdot) \text { converge uniformly to constants } \nu, \mu \text { when } s \rightarrow \infty, \\
& a^{\prime}(s, t+\ell)=O a^{\prime}(s, t), \quad b^{\prime}(s, t+\ell)=O b^{\prime}(s, t), \\
& a_{n}(s, t+\ell)=a_{n}(s, t), \quad b_{n}(s, t+\ell)=b_{n}(s, t) .
\end{aligned}
$$

It will be convenient to notice that this computation did not really involve the precise formula for our Fermi coordinates, but only depended on some of its properties, namely that $\varphi: \mathbb{R} \times(-1,1)^{n-1} \rightarrow L$ is a covering of a neighbourhood of $\gamma$ (so that we can lift everything), and that $g_{i j}=\delta_{i j}+O\left(\left\|x^{\prime}\right\|^{2}\right)$ along $\hat{\gamma}=\mathbb{R} \times\{0\}$. The pseudo-periodicity will soon turn out to be important. This justifies the following. 
Definition 3.13. A map $\varphi: \mathbb{R} \times(-1,1)^{n-1} \rightarrow L$ provides generalized Fermi coordinates near $\gamma$ if $\varphi(t, 0)=\gamma(t),\left(\varphi^{*} g\right)_{i j}\left(x_{n}, x^{\prime}\right)=\delta_{i j}+O\left(\left\|x^{\prime}\right\|^{2}\right)$, and if $\varphi\left(x_{n}+\ell, x^{\prime}\right)=$ $\varphi\left(x_{n}, O x^{\prime}\right)$ for some matrix $O \in O_{n-1}^{+}(\mathbb{R})$ (then $O$ must correspond to the parallel transport along $\left.\gamma_{\mid[0, \ell]}\right)$.

In general, we do not know how to solve the system of equations (8). We now impose some assumptions on the metric that allow us to explicitly compute the solutions and check that the kernel is indeed 1-dimensional.

Proposition 3.14. Let $g$ be a Riemannian metric on $L$ with a closed geodesic $\gamma$. We assume that the metric has the following expansion in some generalized Fermi coordinates:

$$
\hat{g}\left(x_{n}, x^{\prime}\right)=\left(1+k\left\|x^{\prime}\right\|^{2}\right) \delta_{i j}+o\left(\left\|x^{\prime}\right\|^{2}\right), \quad k>0 .
$$

Then the operator $\mathbf{D}_{u_{\gamma, g}}$ is surjective.

Proof: Notice that the coefficients of our partial differential equations depend only on the second derivatives of our metric, so they coincide for two metrics which are tangent of order 2. We can therefore easily compute these coefficients under the assumptions of proposition 3.14 and we find

$$
\frac{\partial^{2} g_{n n}}{\partial x_{i} \partial x_{j}}(t)= \begin{cases}2 k & \text { if } j=i \neq n \\ 0 & \text { else. }\end{cases}
$$

The solutions $z=(a, b)$ of (8) thus satisfy

$$
\begin{array}{rlrl}
\frac{\partial a_{l}}{\partial s}+\frac{\partial b_{l}}{\partial t}-k f(s) a_{l} & =0 & & \forall l \neq n \\
\frac{\partial b_{l}}{\partial s}-\frac{\partial a_{l}}{\partial t}+\frac{1-\chi \circ f(s)}{f(s)} b_{l} & =0 & & \forall l \neq n \\
\frac{\partial b_{n}}{\partial s}-\chi \circ f(s) \frac{\partial a_{n}}{\partial t}-\chi^{\prime} \circ f(s) b_{n} & =0 & \\
\frac{\partial a_{n}}{\partial s}+\frac{1}{\chi \circ f(s)} \frac{\partial b_{n}}{\partial t} & =0 &
\end{array}
$$

We need several changes of variables. First we define $\tilde{a}_{n}:=a_{n}, \tilde{b}_{n}:=\frac{b_{n}}{\chi \circ f}$. Let $\rho(s):=$ $\exp \left(\int_{0}^{s} \frac{1-\chi \circ f\left(s^{\prime}\right)}{f\left(s^{\prime}\right)} d s^{\prime}\right):[0, \infty) \rightarrow \mathbb{R}$ be a solution of the differential equation

$$
\rho^{\prime}(s)=\rho(s) \frac{1-\chi \circ f(s)}{f(s)}, \quad \rho(0)=1,
$$

and put $\tilde{a}_{l}:=\rho(s) a_{l}(s, t)$ and $\tilde{b}_{l}:=\rho(s) b_{l}(s, t)$ for $l=1, \ldots, n-1$.

Since $f(s)$ tends to $\infty$ when $s$ goes to $\infty$ and $\chi(r)=r$ at infinity, we see that $\rho$ and $1 / \chi \circ f$ tend to 0 at $\infty$. As a result, the $\tilde{a}_{l}, \tilde{b}_{l}, \tilde{a}_{n}$ satisfy the same boundary conditions at $s=0, \infty$ as $a_{l}, b_{l}, a_{n}$ for $l \neq n$, but now $\tilde{b}_{n}(0, t) \equiv 0$ and $\tilde{b}_{n}(\infty, t)=0$. Putting also $g(s):=-\frac{\rho^{\prime}}{\rho}(s)-k f(s)$, a straightforward computation shows that these functions satisfy 
now

$$
\begin{array}{lll}
\frac{\partial \tilde{a}_{l}}{\partial s}+\frac{\partial \tilde{b}_{l}}{\partial t}+g(s) \tilde{a}_{l}=0 & \forall l \neq n, & \frac{\partial \tilde{a}_{n}}{\partial s}+\frac{\partial \tilde{b}_{n}}{\partial t}=0 \\
\frac{\partial \tilde{b}_{l}}{\partial s}-\frac{\partial \tilde{a}_{l}}{\partial t}=0 & \frac{\partial \tilde{b}_{n}}{\partial s}-\frac{\partial \tilde{a}_{n}}{\partial t}=0
\end{array}
$$

Let us first focus on our system for $l \neq n$. The functions $\tilde{b}_{l}$ satisfy

$$
\Delta \tilde{b}_{l}+g(s) \frac{\partial \tilde{b}_{l}}{\partial s}=0
$$

together with the pseudo-periodicity and boundary conditions

$$
\tilde{b}_{l}(0)=0, \tilde{b}_{l}(s) \underset{s \rightarrow \infty}{\longrightarrow} 0, \quad \tilde{b}^{\prime}(s, t+\ell)=O \tilde{b}^{\prime}(s, t) .
$$

Since $\Delta h^{2}=2 h \Delta h+2\|\nabla h\|^{2}$, we get $\Delta \tilde{b}_{l}^{2}+g(s) \frac{\partial \tilde{b}_{l}^{2}}{\partial s}=2\left\|\nabla \tilde{b}_{l}\right\|^{2} \geq 0$. Summing these $l$ equations we get

$$
\Delta\left\|\tilde{b}^{\prime}\right\|^{2}+g(s) \frac{\partial\left\|\tilde{b}^{\prime}\right\|^{2}}{\partial s} \geq 0
$$

Define now $h_{\varepsilon}(s, t):=\left\|\tilde{b}^{\prime}\right\|^{2}+\varepsilon e^{-\alpha s-\frac{\beta}{2} t^{2}}$. An immediate computation gives

$$
\Delta h_{\varepsilon}+g(s) \frac{\partial h_{\varepsilon}}{\partial s} \geq \varepsilon\left(\alpha^{2}-\beta+\beta^{2} t^{2}-g(s) \alpha\right) e^{-\alpha s-\frac{\beta}{2} t^{2}} .
$$

We recall that $g(s)=-\frac{\rho^{\prime}}{\rho}(s)-k f(s)=-\frac{1-\chi \circ f}{f}(s)-k f(s)$, that $f(s) \underset{s \rightarrow \infty}{\longrightarrow}+\infty$, and that $\chi(r)=r$ when $r \gg 1$. We therefore see that $-g$ is bounded from below on $[0, \infty)$, so the right hand side of this last inequality is positive if $\alpha$ is large enough, which we assume henceforth. The function $h_{\varepsilon}$ is positive by definition, and does not have any maximum on $[0, \infty) \times \mathbb{R}$ (because its Laplacian is positive at each critical point). Let $\left(s_{n}, t_{n}\right)$ be a maximizing sequence for $h_{\varepsilon}$, that is $h_{\varepsilon}\left(s_{n}, t_{n}\right) \rightarrow \sup h_{\varepsilon}$. Obviously, $s_{n}$ remains bounded because $h_{\varepsilon}$ converges uniformly to 0 as $s$ goes to $\infty$. We also claim that thanks to the quasi-peridodicity $\left(\tilde{b}^{\prime}(s, t+\ell)=O \tilde{b}^{\prime}(s, t)\right)$, we can assume that $t_{n} \in[0, \ell]$. Indeed, if $\operatorname{Frac}\left(\frac{t_{n}}{\ell}\right)$ denotes the fractional part of $\frac{t_{n}}{\ell}$ (whose sign is the same as $t_{n}$ 's), we have

$h_{\varepsilon}\left(s_{n}, t_{n}\right)=\left\|\tilde{b}^{\prime}\left(s_{n}, t_{n}\right)\right\|^{2}+e^{-\alpha s_{n}^{2}-\frac{\beta}{2} t_{n}^{2}}=\left\|\tilde{b}^{\prime}\left(s_{n}, \ell \operatorname{Frac}\left(\frac{t_{n}}{\ell}\right)\right)\right\|^{2}+e^{-\alpha s_{n}^{2}-\frac{\beta}{2} t_{n}^{2}} \leq h_{\varepsilon}\left(s_{n}, \ell \operatorname{Frac}\left(\frac{t_{n}}{\ell}\right)\right)$.

Since $\left(s_{n}, t_{n}\right)$ cannot accumulate at an interior point, we see that $s_{n} \rightarrow 0$, so $\sup h_{\varepsilon}=\varepsilon$. This implies that $h_{\varepsilon} \leq \varepsilon$, so $\left\|\tilde{b}^{\prime}\right\|^{2} \leq \varepsilon$. Since this holds for all $\varepsilon>0$, we conclude that $\tilde{b}^{\prime} \equiv 0$, so $b^{\prime}=0$. Thus, using the second equation of the system (9), we see that $a^{\prime}=a^{\prime}(s)$, and from the first equation, that

$$
\frac{d a^{\prime}(s)}{d s}-k f(s) a^{\prime}(s)=0 .
$$

This last equation gives

$$
a^{\prime}(s)=a^{\prime}(0) e^{k \int_{0}^{s} f(u) d u} .
$$

Letting $s \rightarrow \infty$, and taking into account that $f(s)>0$ and $a^{\prime}(\infty)=0$, we thus get $a^{\prime}(0)=0$, and therefore $a^{\prime}=0$. 
The equations for $\left(\tilde{a}_{n}, \tilde{b}_{n}\right)$ are the standard Cauchy-Riemann equations, so $\tilde{h}(z):=$ $\tilde{a}_{n}(z)+i \tilde{b}_{n}(z)$ is a holomorphic function. By the condition $\tilde{b}_{n}(0, t)=0$, Schwarz reflection provides an extension $h: \mathbb{C} \rightarrow \mathbb{C}$, and in view of the boundary conditions and periodicity, this extension is bounded. By Liouville's theorem, this function $h$ is constant, so $\tilde{b}_{n} \equiv 0$ and $\tilde{a}_{n}$ is a constant. We therefore get that $\operatorname{ker} D$ is one-dimensional, being parameterized by the sole value of the constant $\tilde{a}_{n}=a_{n}$. By proposition $3.10, D$ is surjective.

\subsection{A good Riemannian metric}

The aim of this paragraph is to construct a metric close to the reference metric $g$, which satisfies the assumptions of proposition 3.14. A metric $g^{\prime}$ is said to be $\varepsilon$-close to a metric $g$ if $\forall q \in L$ and $\forall u, v \in T_{q} L,\left|g_{q}(u, v)-g_{q}^{\prime}(u, v)\right| \leq \varepsilon\|u\|_{g}\|v\|_{g}$. This defines a topology on the space of metrics which we call the $\mathcal{C}^{0}$-topology.

Proposition 3.15. Let $g$ be a Riemannian metric on a manifold $L$ and $\beta \in H_{1}(L)$. For all $\varepsilon>0$ there exists a Riemannian metric $g_{\varepsilon, \beta}$ with the following properties:

1. $g_{\varepsilon, \beta}$ is $\varepsilon$-close to $g$ in the $\mathcal{C}^{0}$-topology,

2. $g_{\varepsilon, \beta}$ has a unique minimizing geodesic $\gamma$ in the class $\beta$. Moreover, for some generalized Fermi coordinates $\varphi: \mathbb{R} \times(-1,1)^{n-1} \rightarrow L$ near $\gamma$ and some $k>0, \varphi^{*} g_{\varepsilon, \beta}=(1+$ $\left.k\left\|x^{\prime}\right\|^{2}\right) \delta_{i j}+o\left(\left\|x^{\prime}\right\|^{2}\right)$. In other terms, $\varphi^{*} g_{\varepsilon, \beta}$ is tangent at order 2 to a metric with constant scalar curvature $-k$.

As a result of proposition 3.14 and lemma 3.9, we see that there exists a unique solution to $\mathcal{P}\left(J_{g_{\varepsilon, \beta}}, \beta\right)$. Furthermore the differential of the operator $\bar{\partial}_{J_{g_{\varepsilon, \beta}}}$ is surjective at this solution.

Proof: Since the Riemannian metrics with a unique minimizing geodesic in class $\beta$ are $\mathcal{C}^{l}$-generic (for any $l$ ), we can assume that this uniqueness property holds for $g$. Then the minimizing geodesic $\gamma(\beta)$ is the $k$-cover of some primitive geodesic $\gamma^{\prime}$ in a class $\beta^{\prime}$ such that $k \beta^{\prime}=\beta$ (we say that a geodesic is primitive if it is not a multiple-cover of another one). It is then immediate to check that $\gamma\left(\beta^{\prime}\right)=\gamma^{\prime}$. In dimension 2 , a primitive geodesic that minimizes the length in a homology class is always simple (i.e. injective). In dimension higher than 2 , the property of a primitive geodesic being simple is $\mathcal{C}^{l}$-generic (in the metric), so a further perturbation of $g$ ensures that $\gamma^{\prime}$ is simple. Summarizing this preparatory discussion, we can slightly perturb $g$ so as to ensure that $\gamma:=\gamma(\beta)=\gamma\left(k \beta^{\prime}\right)$ for some $k \geq 1$ is the unique $\beta$-minimizing geodesic, while $\gamma^{\prime}:=\gamma\left(\beta^{\prime}\right)$ is a simple closed geodesic. We denote $\ell^{\prime}$ its length and let $\varphi: \mathbb{R} \times(-1,1)^{n-1} \rightarrow L$ be Fermi coordinates near $\gamma^{\prime}$. Then,

$$
\varphi^{*} g=\delta_{i j}+h_{i j}\left(x_{n}, x^{\prime}\right), \quad\left|h_{i j}\left(x_{n}, x^{\prime}\right)\right| \leq C\left(x_{n}\right)\left\|x^{\prime}\right\|^{2} .
$$

The pseudo-periodicity of $\varphi$ has some immediate consequences. 
- $\varphi_{*}\left\|x^{\prime}\right\|$ is a well-defined function locally near $\gamma^{\prime}$ on $L$. With slight abuse of notation, we therefore understand $\left\|x^{\prime}\right\|$ as a function defined on both $\mathbb{R} \times(-1,1)^{n-1}$ and $L$ (near $\left.\gamma^{\prime}\right)$.

- Similarly, $\varphi_{*} \delta_{i j}$ is a well-defined tensor locally near $\gamma^{\prime}$ on $L$, because the ambiguity in multi-valuedness of $\varphi$ is given by an orthogonal matrix $O$ in the fiber direction, whose derivative is again $O$, which precisely preserves the metric $\delta_{i j}$. By construction of the Fermi coordinates, $g=\varphi_{*} \delta_{i j}+O\left(\left\|x^{\prime}\right\|^{2}\right)$.

- $h_{i j}\left(x_{n}+\ell^{\prime}, x^{\prime}\right)=h_{i j}\left(x_{n}, O x^{\prime}\right)$, so our bound on $h_{i j}$ is in fact uniform in $x_{n}:\left|h_{i j}\left(x_{n}, x^{\prime}\right)\right|$ $\leq C\left\|x^{\prime}\right\|^{2}$ for some constant $C$ large enough.

Let now $\rho:[0, \infty) \rightarrow[0,1]$ be a smooth non-increasing function that equals 1 near 0 , with Supp $\rho=[0,1]$, and define $\rho_{\varepsilon}(t):=\rho(t / \varepsilon)$. The previous remarks show that

$$
g_{\varepsilon}:=\rho_{\varepsilon}\left(\left\|x^{\prime}\right\|^{2}\right)\left(1+n C\left\|x^{\prime}\right\|^{2}\right) \varphi_{*} \delta_{i j}+\left(1-\rho_{\varepsilon}\left(\left\|x^{\prime}\right\|^{2}\right)\right)(1+\varepsilon) g
$$

is a well-defined metric on $L$. We claim that it has the required properties provided $\varepsilon$ is chosen small enough. Indeed, since both $g$ and $\left(1+n C\left\|x^{\prime}\right\|^{2}\right) \varphi_{*} \delta_{i j}$ are tangent to $\varphi_{*} \delta_{i j}$, the difference $\left\|g-g_{\varepsilon}\right\|_{\mathcal{C}^{0}}$ is of order $\varepsilon$ for $\varepsilon$ small enough, so point (1) of proposition 3.15 holds. Next, we prove that $\gamma$ is the unique $\beta$-minimizing geodesic for $g_{\varepsilon}$. Notice that

$$
\begin{aligned}
\varphi^{*} g_{\varepsilon}-\varphi^{*} g & =\rho_{\varepsilon}\left(\left\|x^{\prime}\right\|^{2}\right)\left(\left(1+n C\left\|x^{\prime}\right\|^{2}\right) \delta_{i j}-\left(\delta_{i j}+h_{i j}\left(x_{n}, x^{\prime}\right)\right)\right)+\left(1-\rho_{\varepsilon}\left(\left\|x^{\prime}\right\|^{2}\right)\right) \varepsilon g \\
& \geq \rho_{\varepsilon}\left(\left\|x^{\prime}\right\|^{2}\right)\left(n C\left\|x^{\prime}\right\|^{2} \delta_{i j}-h_{i j}\left(x_{n}, x^{\prime}\right)\right)
\end{aligned}
$$

and the estimate $\left|h_{i j}\left(x_{n}, x^{\prime}\right)\right| \leq C\left\|x^{\prime}\right\|^{2}$ holds. Thus, $\varphi^{*} g_{\varepsilon} \geq \varphi^{*} g$ for $\varepsilon$ small enough, with equality if and only if $x^{\prime}=0$. Thus, $g_{\varepsilon} \geq g$ in the neighbourhood of $\operatorname{Im} \gamma^{\prime}=\operatorname{Im} \gamma$ endowed with our Fermi coordinates, with equality exactly on $\operatorname{Im} \gamma$. On the other hand, if $\varepsilon$ is small enough, $g_{\varepsilon}=(1+\varepsilon) g$ outside this neighbourhood. Thus we see that $g_{\varepsilon} \geq g$ on $L$, with equality exactly on $\operatorname{Im} \gamma$. Then $\ell_{g_{\varepsilon}}(\gamma)=\ell_{g}(\gamma)=\ell$, while if $\gamma_{2}$ is any other closed connected curve in the class $\beta, \ell_{g_{\varepsilon}}\left(\gamma_{2}\right)>\ell_{g}\left(\gamma_{2}\right) \geq \ell$. We conclude that $\gamma$ is indeed the unique $g_{\varepsilon}$-minimizing geodesic in the class $\beta$. Moreover, in the multi-valued coordinates $\varphi$ near $\gamma$, we have $\varphi^{*} g_{\varepsilon}=\left(1+n C\left\|x^{\prime}\right\|^{2}\right) \delta_{i j}$. Finally, notice that although $\varphi$ does not provide Fermi coordinates for $g_{\varepsilon}$ near $\gamma^{\prime}$, it does provide generalized Fermi coordinates because $\varphi\left(x_{n}+\ell^{\prime}, x^{\prime}\right)=\varphi\left(x_{n}, O x^{\prime}\right)$ and $\varphi^{*} g_{\varepsilon}$ is indeed tangent to $\delta_{i j}$ at order 2 (see definition 3.13). Point (2) therefore holds for $g_{\varepsilon}$.

Remark 3.16. We have proved more than stated. In fact, we see that we can even choose a minimizing geodesic $\gamma$ for $g$ and construct a deformation of $g$ that achieves the conditions of proposition 3.15, for which $\gamma$ remains a geodesic for the parameter of the deformation. This point is not really important in our argument. It simply allows for an easy and explicit computation of the index of the operator $\mathbf{D}_{u_{\gamma, g}}$ (corollary A.7). 


\subsection{Somewhere injectivity}

We recall the context first. $(L, g)$ is a Riemannian manifold, $J \in \mathcal{J}_{\mathrm{Cyl}, g}^{\infty}$ is an almost complex structure on $T^{*} L, \beta \in H_{1}(L)$ is a class with a unique minimal representative, which has a primitive geodesic (i.e. not a multiple cover). This last assumption is essential in this section. Let $\mathcal{M}(J, \beta)$ be the set of $J$-holomorphic maps from the punctured disc to $T^{*} L$, asymptotic at 0 to a lift of the minimal geodesic in the class $\beta$ and with boundary on $L$ (see the precise definition on p. 8). Recall that a map $u: D \backslash\{0\} \rightarrow T^{*} L$ is said to be somewhere injective in a region $K \subset T^{*} L$ if there exists a point $z \in D \backslash\{0\}$ with $u(z) \in$ int $K$ and $u^{-1}(u(z))=\{z\}$. We call such a point an injectivity point. The aim of this section is to show that all elements of the moduli space $\mathcal{M}(J, \beta)$ have a somewhere injective point in a region where we will be free to vary our almost complex structure.

Lemma 3.17 (Somewhere injectivity). For any $0<r<R<\infty$ every element $u \in \mathcal{M}(J, \beta)$ has an injectivity point in $T_{R}^{*} L \backslash T_{r}^{*} L$.

Proof: Let $u:(D \backslash\{0\}, \partial D) \rightarrow\left(T^{*} L, L\right)$ be a $J$-holomorphic map asymptotic to the lift of the geodesic $\gamma(\beta)$, which is primitive by assumption. Notice that this lift is then automatically injective. Let $0<\varepsilon<r$ be a regular value of $\|u\|_{g}$, so that the subset $\left\{\|u\|_{g}=\varepsilon\right\} \subset D \backslash\{0\}$ is a finite union of closed circles. Let $\Omega$ be the connected component of a neighbourhood of 0 in $\left\{\|u\|_{g}>\varepsilon\right\}$ and $\Omega_{1}, \ldots, \Omega_{n}$ the other components (there are finitely many). Let $v:=u_{\mid \Omega}$ and $v_{i}:=u_{\mid \Omega_{i}}$. Note that since $u(\partial D) \subset L$, none of these components touches $\partial D$.

Somewhere injectivity of $v . \quad v$ is a proper $J$-holomorphic map $\Omega \rightarrow T^{*} L \backslash T_{\varepsilon}^{*} L$. We define the following subsets of $\Omega$,

$$
\begin{aligned}
& \mathcal{I}(v):=\left\{z \mid v^{-1}(v(z))=\{z\}\right\}, \\
& \mathcal{C}(v):=\{z \mid d v(z)=0\}, \\
& \mathcal{D}(v):=\left\{z \mid \exists z^{\prime} \neq z, v(z)=v\left(z^{\prime}\right) \text { and } v(\operatorname{Op}(z)) \neq v\left(\operatorname{Op}\left(z^{\prime}\right)\right)\right\} .
\end{aligned}
$$

Another description of $\mathcal{D}(v)$ is that the image of the restriction of $v$ to any two neighbourhoods of $z$ and $z^{\prime}$ never coincides (this set corresponds to the points where distinct branches of $v$ intersect). It is well-known that Micaleff-White's theorem implies that the sets $\mathcal{C}(v)$ and $\mathcal{D}(v)$ are discrete (in fact $\mathcal{C}(v)$ is even finite in our situation) [MW95, MS12]. Moreover, we have the following standard fact.

Claim 3.18. $\mathcal{I}(v) \backslash \mathcal{C}(v)$ is open and $\mathcal{I}(v) \cup \mathcal{D}(v)$ is closed.

Proof: We first prove that $\mathcal{I}(v) \backslash \mathcal{C}(v)$ is open in $\Omega$, hence that ${ }^{c} \mathcal{I}(v) \cup \mathcal{C}(v)$ is closed. Since $\mathcal{C}(v)$ is discrete, from a sequence of points $z_{n}$ in the complement of $\mathcal{I}(v) \backslash \mathcal{C}(v)$ that converges to a point $z \in \Omega$, we can either extract a constant subsequence in $\mathcal{C}(v)$ or a subsequence in ${ }^{c} \mathcal{I}(v)$. In the first case, $z \in \mathcal{C}(v)$ and we are done. So we assume henceforth the latter and we replace $z_{n}$ by its subsequence, so $z_{n} \notin \mathcal{I}(v) \forall n$. Thus, there exist points $z_{n}^{\prime} \neq z_{n}$ in $\Omega$ such that $v\left(z_{n}\right)=v\left(z_{n}^{\prime}\right)$. Since $v$ is proper, continuous, and $v\left(z_{n}\right)$ converges, we can extract

further so that $z_{n}^{\prime} \rightarrow z^{\prime} \in \Omega$, with $v(z)=v\left(z^{\prime}\right)$. If $z \neq z^{\prime}$, these points do not belong to $\mathcal{I}(v)$, and if $z=z^{\prime}, v$ is injective in no neighbourhood of $z$, so $z \in \mathcal{C}(v)$. 
To see that $\mathcal{I}(v) \cup \mathcal{D}(v)$ is closed, it is enough to prove that $\overline{\mathcal{I}(v)} \subset \mathcal{I}(v) \cup \mathcal{D}(v)$ (because $\mathcal{D}(v)$ is discrete). Consider a sequence $z_{n} \in \mathcal{I}(v)$ that converges to a point $z \in \Omega \backslash \mathcal{I}(v)$. Then, there exists $z^{\prime} \neq z$ such that $v\left(z^{\prime}\right)=v(z)$, and since $z_{n} \in \mathcal{I}(v), v\left(\mathrm{Op}\left(z^{\prime}\right)\right)$ cannot coincide with $v(\mathrm{Op}(z))$. This finishes the proof of our claim.

Since $\mathcal{C}(v), \mathcal{D}(v)$ are discrete their union is countable. So there exists a smooth embedded path $\gamma \subset D$ emanating from the origin, joining $\partial D$, that avoids $\mathcal{C}(v)$ and $\mathcal{D}(v)$ and such that $\gamma \cap \Omega$ is connected. It is then clear that $\mathcal{I}(v) \cap \gamma \cap \Omega$ is both open and closed in $\gamma \cap \Omega$. Now since $v$ is asymptotic at 0 to the lift of a primitive geodesic, which is injective as we noticed already, a neighbourhood of 0 in $\Omega$ is contained in $\mathcal{I}(v)$. We therefore conclude that $\gamma \cap \Omega \subset \mathcal{I}(v)$ and by the intermediate value theorem there exists an element $z \in \mathcal{I}(v)$ with $v(z)=u(z) \in T_{R}^{*} L \backslash T_{r}^{*} L$ (recall that $\|v(z)\|_{g}$ goes to $\infty$ when $z$ tends to 0 and goes to $\varepsilon<r$ when $z$ tends to $\partial \Omega)$. In fact, there exists even a connected open sub-arc $\gamma^{\prime} \subset \gamma \cap \Omega \subset \mathcal{I}(v)$, whose image by $v$ lies in $T_{R}^{*} L \backslash T_{r}^{*} L$.

Somewhere injectivity of $u$. We now conclude our proof by showing that some point of $\gamma^{\prime}$ is a point of injectivity for $u$ itself. We argue by contradiction. Assume that this is not the case, then for every $z \in \gamma^{\prime}$ there exists $j \in\{1, \ldots, n\}$ and $\xi_{j} \in \Omega_{j}$ such that $v(z)=v_{j}\left(\xi_{j}\right)$. In particular for all $j$ the subsets $\left\{z \in \gamma^{\prime} \mid \exists \xi_{j} \in \Omega_{j}: v(z)=v_{j}\left(\xi_{j}\right)\right\}$ are closed in $\gamma^{\prime}$. Indeed, if $z_{n} \in \gamma^{\prime} \rightarrow z \in \gamma^{\prime}$ is such that $\exists \xi_{n} \in \Omega_{j}$ with $v\left(z_{n}\right)=v_{j}\left(\xi_{n}\right)$, then by properness of $v_{j}, \xi_{n}$ has a subsequence converging to $\xi_{*} \in \Omega_{j}$ and we have $v(z)=v_{j}\left(\xi_{*}\right)$.

Since $\gamma^{\prime}$ is open in $\gamma \cap \Omega$, this is only possible if one of these subsets contains an open sub-arc of $\gamma^{\prime}$. Thus, replacing $\gamma^{\prime}$ by this sub-arc if needed, one of the maps $v_{j}$ (say $v_{1}$ ) must satisfy $\operatorname{Im} v_{1} \supset v\left(\gamma^{\prime}\right)$. Let $\Omega^{\prime}$ be the connected component of $\gamma^{\prime}$ in the set of points $z \in \Omega$ such that $v(z)=v_{1}\left(z^{\prime}\right)$ for some $z^{\prime} \in \Omega_{1}$. Then, by the same argument as above, $\Omega^{\prime}$ is closed in $\Omega$ by properness of $v_{1}$. Notice that $\operatorname{since} \mathcal{C}(v)$ is finite, $\Omega^{\prime} \backslash \mathcal{C}(v)$ is also closed in $\Omega \backslash \mathcal{C}(v)$. We claim that $\Omega^{\prime} \backslash \mathcal{C}(v)$ is also open in $\Omega \backslash \mathcal{C}(v)$. Indeed, if $z \in \Omega^{\prime} \backslash \mathcal{C}(v)$, there is by definition a sequence of distinct points $z_{n} \in \Omega^{\prime}$ that converges to $z$ (because $\Omega^{\prime}$ is the connected component of an open arc). Notice that the Micaleff-White theorem shows that the preimage of any point $v\left(z_{n}\right)$ is finite, so we can assume that the $v\left(z_{n}\right)$ are distinct as well. By definition of $\Omega^{\prime}$, there are distinct points $z_{n}^{\prime} \in \Omega_{1}$ such that $v_{1}\left(z_{n}^{\prime}\right)=v\left(z_{n}\right) \rightarrow v(z)$. Now since $z \in \Omega$, we have $\|v(z)\|_{g}>\varepsilon$, and since $v_{1}$ is proper into $\left\{\|p\|_{g}>\varepsilon\right\}$, the sequence $z_{n}^{\prime}$ is compactly contained in $\Omega_{1}$. Thus, we can assume that $z_{n}^{\prime}$ converges to $z^{\prime} \in \Omega_{1}$. Then, $v(z)=v_{1}\left(z^{\prime}\right)$, and using [MS12, Lemma 2.4.3] $(z \notin \mathcal{C}(v))$, we get that $z \in \operatorname{int} \Omega^{\prime} \backslash \mathcal{C}(v)$. Thus $\Omega^{\prime} \backslash \mathcal{C}(v)$ is open and closed in $\Omega \backslash \mathcal{C}(v)$, which is connected, so $\Omega^{\prime} \backslash \mathcal{C}(v)=\Omega \backslash \mathcal{C}(v)$. Since $\Omega^{\prime}$ is also closed in $\Omega$, we get that $\Omega^{\prime}=\Omega$. But this is impossible, since $\left\|v_{1}\right\|_{g}$ is bounded while $\|v\|_{g}$ is not. 


\subsection{Compactness results for punctured holomorphic disks}

We state compactness results for sequences of punctured holomorphic disks in cotangent bundles. In this section we will use results from symplectic field theory and various constructions therein, such as the notion of holomorphic buildings and the splitting of symplectic manifolds along a contact hypersurface (stretching the neck). We give details where possible, but for the sake of the exposition we refer the reader to $\left[\mathrm{BEH}^{+} 03\right]$ and [Abb14] for the precise definitions and notions in symplectic field theory. In order to make this section more readable we adapt the notation to the setting of punctured disks in cotangent bundles, even though the results may hold in more general settings.

Our setting is as follows. For a closed Riemannian manifold $(L, g)$ we denote by $M \subset$ $T^{*} L$ the unit cotangent bundle and by $\mathcal{W}_{g}$ the open unit disk bundle, i.e. $\partial \mathcal{W}_{g}=M$. Then $\left(M, \alpha:=\left.\lambda\right|_{M}\right)$ is a contact manifold and $\left(T^{*} L, d \lambda\right)$ has a cylindrical end $E$ given by $\left(E:=T^{*} L \backslash \mathcal{W}_{g}, d \lambda\right) \simeq([1, \infty) \times M, d(r \alpha))$. As before we denote by $L \subset T^{*} L$ the zero-section and note that the above identification extends to $\left(T^{*} L \backslash L, d \lambda\right) \simeq((0, \infty) \times M, d(r \alpha))$.

\subsubsection{Energy of holomorphic curves in cotangent bundles}

Let $J \in \mathcal{J}_{\mathrm{cyl}, g}^{\infty}$ be an almost complex structure on $T^{*} L$ that is cylindrical on the whole end $E$. Let $(S, j)$ be a compact Riemann surface (possibly with boundary) and let $Z \subset S$ be a finite subset of interior punctures. For a $J$-holomorphic map $F:(S \backslash Z, j) \rightarrow\left(T^{*} L, J\right)$ we write $F=(a, f): U \subset S \backslash Z \rightarrow[1, \infty) \times M$ on the subset $U:=F^{-1}(E)$. In $\left(T^{*} L, \omega:=d \lambda\right)$ we define the $\omega$-energy of $F$ as

$$
\mathcal{E}_{\omega}(F)=\int_{F^{-1}\left(\mathcal{W}_{g}\right)} F^{*} \omega+\int_{F^{-1}(E)} f^{*} d \alpha .
$$

Let $\Lambda:=\left\{\varphi \in C_{c}^{\infty}([1, \infty)) \mid \varphi \geq 0, \int_{1}^{\infty} \varphi(s) d s=1\right\}$. We define the $\alpha$-energy to be

$$
\mathcal{E}_{\alpha}(F)=\sup _{\varphi \in \Lambda} \int_{F^{-1}(E)} \varphi \circ a d a \wedge f^{*} \alpha
$$

The total energy of $F$ is then defined as the sum

$$
\mathcal{E}(F)=\mathcal{E}_{\omega}(F)+\mathcal{E}_{\alpha}(F) .
$$

In order to apply the SFT compactness theorem we must find a uniform upper bound on the energy of a sequence of punctured holomorphic curves. Compared with the lemmas of these kind present in $\left[\mathrm{BEH}^{+} 03\right]$, for instance proposition 6.3 , the following estimation allows us to consider a sequence of almost complex structures that are all cylindrical, but not fixed at infinity.

Lemma 3.19. Let $J$ be an almost complex structure on $T^{*} L$ that is cylindrical on the end $E$. Let $\gamma$ be a closed unit speed geodesic on $L$ and $\tilde{\gamma}$ its lift to $M$. For every J-holomorphic curve $u:(D \backslash\{0\}, \partial D) \rightarrow\left(T^{*} L, L\right)$ asymptotic to $\tilde{\gamma}$ at 0 we have

$$
\mathcal{E}(u) \leq 3 \alpha(\tilde{\gamma})=3 \ell_{g}(\gamma)
$$


Proof: Let $u:(D \backslash\{0\}, \partial D) \rightarrow\left(T^{*} L, L\right)$ be a $J$-curve asymptotic to $\tilde{\gamma}$ at 0 . Recall that $T^{*} L=\mathcal{W}_{g} \cup E$, where $E \simeq([1, \infty) \times M, d(r \alpha))$ and $\mathcal{W}_{g} \backslash L \simeq((0,1) \times M, d(r \alpha))$, that $J$ is $d \lambda$-compatible on $T^{*} L$ and compatible with $\alpha$ on $E$. These compatibilities imply that $d \alpha(\cdot, J \cdot) \geq 0$ and $d r \wedge \alpha(\cdot, J \cdot) \geq 0$ on $E_{-\delta}:=[1-\delta, \infty) \times M$ for some $\delta>0$ small enough. We will exploit this non-negativity below, under the wording that $\alpha$ is tamed by $J$.

On $u^{-1}\left(E_{-\delta}\right)$ we put $u=(a, \tilde{u}) \in[1-\delta, \infty) \times M$. The total energy of $u$ is then given by $\mathcal{E}(u)=\sup \left\{\mathcal{E}_{\varphi}(u) \mid \varphi \in \Lambda\right\}$, where

$$
\mathcal{E}_{\varphi}(u)=\int_{u^{-1}\left(\mathcal{W}_{g}\right)} u^{*} \omega+\int_{u^{-1}(E)} \tilde{u}^{*} d \alpha+\varphi \circ a d a \wedge \tilde{u}^{*} \alpha .
$$

Let us fix $\varphi \in \Lambda$ (thus $\varphi:[1, \infty) \rightarrow[0, \infty)$ has compact support and $\left.\int_{1}^{\infty} \varphi=1\right)$ and consider a smooth function $\tilde{\varphi}:(0, \infty) \rightarrow[0, \infty)$ such that $\tilde{\varphi}(t)=1$ for $t \in(0,1-\delta], \tilde{\varphi}(t) \geq 1$ for $t \leq 1, \tilde{\varphi}(t) \geq \varphi(t)$ for $t>1, \tilde{\varphi}=0$ near $\infty$, and $\int_{0}^{\infty} \tilde{\varphi} \leq 3$ (this is possible because $\left.\int_{0}^{1} 1 d t=\int_{1}^{\infty} \varphi(t) d t=1\right)$. Define then $h(t):=\int_{0}^{t} \tilde{\varphi}(s) d s$ and $\omega_{h}:=d(h \alpha)$. Since $h(t)=t$ for $t<1-\delta, \omega_{h}$ coincides with $\omega=d \lambda$ on $(0,1-\delta] \times M$ and in particular extends to the zero section. We claim that

$$
\begin{aligned}
0 & \leq u^{*} \omega_{h}, \\
\mathcal{E}_{\varphi}(u) & \leq \int u^{*} \omega_{h} .
\end{aligned}
$$

Let us first see how to conclude. By (13) we have

$$
\int_{D \backslash\{0\}} u^{*} \omega_{h}=\lim _{\varepsilon \rightarrow 0} \int_{D \backslash D_{\varepsilon}} u^{*} \omega_{h}=\lim _{\varepsilon \rightarrow 0} \int_{D \backslash D_{\varepsilon}} u^{*} d(h \alpha)=-\lim _{\varepsilon \rightarrow 0} \int_{\partial D_{\varepsilon}} h \circ a \tilde{u}^{*} \alpha .
$$

Since $h$ is constant near $\infty, h(\infty) \leq 3$ and $u$ is asymptotic to $\tilde{\gamma}$ at 0 . Note that the orientation of $\partial D_{\varepsilon}$ corresponds to $-\tilde{\gamma}:=\tilde{\gamma}(-t)$ in the limit as $\varepsilon \rightarrow 0$ (see p.12). Now using (14) we get

$$
\mathcal{E}_{\varphi}(u) \leq \int u^{*} \omega_{h}=-h(\infty) \int_{-\tilde{\gamma}} \alpha=h(\infty) \alpha(\tilde{\gamma}) \leq 3 \ell_{g}(\gamma)
$$

Since this holds for all $\varphi$, we have our desired bound on $\mathcal{E}(u)$.

It remains to establish our claim. For (13), notice that $\omega_{h}=d \lambda$ on $(0,1-\delta] \times M$, so $u^{*} \omega_{h} \geq 0$ on $u^{-1}(L \cup(0,1-\delta) \times M)$. On $[1-\delta, \infty) \times M, \alpha$ being tamed by $J$, it is easy to see that $\tilde{u}^{*} d \alpha \geq 0$ and $d a \wedge \tilde{u}^{*} \alpha \geq 0$. Thus, since $h^{\prime} \geq 0$, we get

$$
u^{*} \omega_{h}=u^{*} d(h \alpha)=h(a) \tilde{u}^{*} d \alpha+h^{\prime}(a) d a \wedge \tilde{u}^{*} \alpha \geq 0 .
$$

We now prove (14).

$$
\begin{aligned}
\mathcal{E}_{\varphi}(u)= & \int_{u^{-1}\left(\mathcal{W}_{g}\right)} u^{*} \omega+\int_{u^{-1}(E)}\left(\tilde{u}^{*} d \alpha+\varphi \circ a d a \wedge \tilde{u}^{*} \alpha\right) \\
= & \int_{u^{-1}\left(\mathcal{W}_{g} \backslash E_{-\delta}\right)} u^{*} \omega+\int_{u^{-1}\left(\mathcal{W}_{g} \cap E_{-\delta}\right)} u^{*} d(r \alpha)+\int_{u^{-1}(E)}\left(\tilde{u}^{*} d \alpha+\varphi \circ a d a \wedge \tilde{u}^{*} \alpha\right) \\
= & \int_{u^{-1}\left(\mathcal{W}_{g} \backslash E_{-\delta}\right)} u^{*} \omega_{h}+\int_{u^{-1}\left(\mathcal{W}_{g} \cap E_{-\delta}\right)}\left(a \tilde{u}^{*} d \alpha+d a \wedge \tilde{u}^{*} \alpha\right) \\
& +\int_{u^{-1}(E)}\left(\tilde{u}^{*} d \alpha+\varphi \circ a d a \wedge \tilde{u}^{*} \alpha\right)
\end{aligned}
$$


As we already noticed, since $u$ is $J$-holomorphic for a $J$ which tames $\alpha$ on $E_{-\delta}$, we have $\tilde{u}^{*} d \alpha \geq 0$ and $d a \wedge \tilde{u}^{*} \alpha \geq 0$, so the inequalities $h(t) \geq t$ and $h^{\prime}(t) \geq 1$ for $0<t<1, h(t) \geq 1$ for $t \geq 1, h^{\prime}(t) \geq \varphi$ on $t \geq 1$ imply that

$$
\begin{aligned}
\mathcal{E}_{\varphi}(u) \leq & \int_{u^{-1}\left(\mathcal{W}_{g} \backslash E_{-\delta}\right)} u^{*} \omega_{h}+\int_{u^{-1}\left(\mathcal{W}_{g} \cap E_{-\delta}\right)}\left(h(a) \tilde{u}^{*} d \alpha+h^{\prime}(a) d a \wedge \tilde{u}^{*} \alpha\right) \\
& +\int_{u^{-1}(E)}\left(h(a) \tilde{u}^{*} d \alpha+h^{\prime}(a) d a \wedge \tilde{u}^{*} \alpha\right) \\
\leq & \int_{u^{-1}\left(\mathcal{W}_{g} \backslash E_{-\delta}\right)} u^{*} \omega_{h}+\int_{u^{-1}\left(E_{-\delta}\right)} u^{*} d(h \alpha)=\int u^{*} \omega_{h} .
\end{aligned}
$$

This concludes the proof of (14) and thus of our lemma.

If $J$ is only compatible with $\alpha$ on $[K, \infty) \times M$ for a constant $K>1$, a rescaling provides the estimate $\mathcal{E}(u) \leq 3 K \ell_{g}(\gamma)$. Thus lemma 3.19 implies the following statement.

Corollary 3.20. Let $J_{n}$ be a sequence of almost complex structures in $\mathcal{J}_{\mathrm{Cyl}, g}^{\infty}$ which are compatible with $\alpha$ outside a fixed compact set. Then there exists a constant $C>0$ that depends only on the fixed compact set, such that every $J_{n}$-holomorphic curve $u_{n}:(D \backslash\{0\}, \partial D) \rightarrow$ $\left(T^{*} L, L\right)$ asymptotic to $\tilde{\gamma}$ at 0 satisfies the energy bound $\mathcal{E}\left(u_{n}\right) \leq C$.

\subsubsection{Compactness for punctured holomorphic disks I}

Our first compactness result concerns sequences of $J_{n}$-holomorphic curves, where $J_{n} \in \mathcal{J}_{\mathrm{Cyl}, g}^{\infty}$ converge to some $J \in \mathcal{J}_{\mathrm{Cyl}, g}^{\infty}$.

Theorem 3.21. Let $(L, g)$ be a closed Riemannian manifold which has a unique minimizing geodesic $\gamma$ in the class $\beta \in H_{1}(L ; \mathbb{Z})$. Let $J_{n} \in \mathcal{J}_{\mathrm{Cyl}, g}^{\infty}$ be a sequence of almost complex structures on $T^{*} L$ that are fixed outside a compact subset and converge to an almost complex structure $J \in J_{\mathrm{Cyl}, g}^{\infty}$ in the $C^{\infty}$-topology. Assume $u_{n}:(D \backslash\{0\}, \partial D) \rightarrow\left(T^{*} L, L\right)$ is a sequence of $J_{n}$-holomorphic curves asymptotic to $\tilde{\gamma}$ at 0 . Then, after restricting to a subsequence, $u_{n}$ converges in $C_{\mathrm{loc}}^{\infty}$ to a J-holomorphic map $u:(D \backslash\{0\}, \partial D) \rightarrow\left(T^{*} L, L\right)$ asymptotic to $\tilde{\gamma}$ at 0 .

Proof: Since all $u_{n}$ are asymptotic to $\tilde{\gamma}$ at 0 , by corollary 3.20 the energy $\mathcal{E}\left(u_{n}\right)$ is uniformly bounded. We now apply the SFT compactness theorem for punctured holomorphic curves with boundary in symplectic cobordisms $\left[\mathrm{BEH}^{+} 03, \mathrm{Abb} 14\right]$. It implies that there exists $N \in$ $\mathbb{N}$ such that our sequence $u_{n}:(D \backslash\{0\}, \partial D) \rightarrow\left(T^{*} L, L\right)$ converges to a stable holomorphic building of height $N$. This is given by the following data in our situation.

(i) $v_{0}:\left(S_{0} \backslash Z_{0}, \partial S_{0}, j_{0}\right) \rightarrow\left(T^{*} L, L, J\right)$ is a proper $J$-holomorphic map from a compact Riemann surface with boundary $S_{0}$ with a finite set of punctures $Z_{0} \subset S_{0} \backslash \partial S_{0}$ to the almost complex manifold $\left(T^{*} L, J\right)$ such that $v_{0}\left(\partial S_{0}\right) \subset L$ and $v_{0}$ has finite energy. Note that $S_{0}$ may have multiple components. 
(ii) For $k=1, \ldots, N$ we have holomorphic maps $v_{k}:\left(S_{k} \backslash Z_{k}, j_{k}\right) \longrightarrow(\mathbb{R} \times M, \widehat{J})$ from closed Riemann surfaces $S_{k}$ with a finite set of punctures $Z_{k} \subset S_{k}$ to the symplectization $\mathbb{R} \times M$. Here the almost complex structure $\widehat{J}$ is $\mathbb{R}$-invariant and coincides with $J$ in the region of $[1, \infty) \times M$ where $J$ is translation invariant. Furthermore we have decoration maps $\Phi_{k}$ for $k=1, \ldots, N$ that glue all of the negative punctures of $Z_{k}$ to the positive punctures of $Z_{k-1}$. More precisely, let $\bar{S}_{k}$ denote the oriented blow-up of $S_{k}$ at the points $z \in Z_{k}$. The conformal action $j_{k}$ defines a circle action at every boundary circle of $\bar{S}_{k}$ and a decoration $\Phi_{k}$ is a choice of map from the negative boundary circles of $\bar{S}_{k}$ to the positive boundary circles of $\bar{S}_{k-1}$ that anti-commutes with the circle action $\left(\Phi_{k}\left(e^{i t} \xi\right)=e^{-i t} \Phi_{k}(\xi)\right)$.

(iii) Denote by $\bar{S}:=\bar{S}_{0} \cup_{\Phi_{1}} \bar{S}_{1} \cup \ldots \cup_{\Phi_{N}} \bar{S}_{N}$ the piecewise smooth surface obtained by gluing together all blow ups $\bar{S}_{k}$ of $S_{k}$ at their punctures via the decoration maps $\Phi_{k}$. Then $\bar{S}$ has no nodal points and is homeomorphic to $\widehat{D_{0}}:=\mathbb{R}_{\geq 0} \times S^{1} \cup\{\infty\} \times S^{1}$, which is homeomorphic to the oriented blow-up of $D$ at 0 . This holds because $T^{*} L$ and $\mathbb{R} \times M$ are exact symplectic manifolds, so there can be neither bubbling of spheres, nor of discs with boundary on $L$ (in a more general context with possible bubbling, this point holds only if we add nodal points to our discussion). Furthermore there exists a diffeomorphism $G: T^{*} L=\overline{\mathcal{W}}_{g} \cup_{M} E \rightarrow \mathcal{W}_{g}$ such that the compositions $G \circ v_{0}$ and $\pi \circ v_{k}$ for all $k$ fit together into a continuous map $\bar{S} \rightarrow \overline{\mathcal{W}}_{g}$ that maps $\{\infty\} \times S^{1}$ to $\tilde{\gamma}$, where $\pi: \mathbb{R} \times M \rightarrow M$ is the projection.

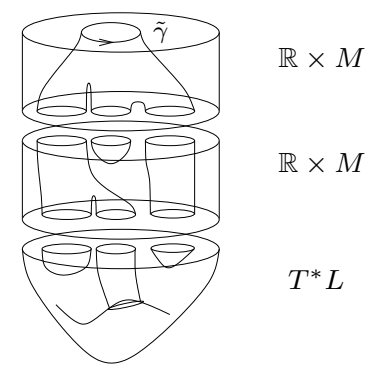

Figure 1: A holomorphic building appearing as a limit of $u_{n}$

We now analyze the limit holomorphic building. We start by considering the top floor of the building, i.e. the holomorphic curve given by $v_{N}:\left(S_{N} \backslash Z_{N}, j_{N}\right) \longrightarrow(\mathbb{R} \times M, \widehat{J})$. By point (iii) the piecewise smooth surface $\bar{S}$ is homeomorphic to $\widehat{D_{0}}$, hence only one component of $S_{N}$ has a positive puncture in $Z_{N}$. This implies that there are no other components of $S_{N}$, since the maximum principle asserts that every punctured holomorphic curve in $\mathbb{R} \times M$ must have at least one positive puncture. The genus of $\bar{S}$ is 0 , thus we have $S_{N}=\mathbb{C} P^{1}, Z_{N}=$ $\left\{z_{0}, z_{1}, \ldots, z_{l}\right\}$ is a finite set of punctures and $v_{N}:\left(\mathbb{C} P^{1} \backslash\left\{z_{0}, z_{1}, \ldots, z_{l}\right\}, j_{N}\right) \rightarrow(\mathbb{R} \times M, \widehat{J})$ is a $\widehat{J}$-holomorphic curve asymptotic to the Reeb orbit $\tilde{\gamma}$ at the positive puncture, say $z_{0}$, and asymptotic to Reeb orbits $\tilde{\gamma}_{1}, \ldots, \tilde{\gamma}_{l}$ at the negative punctures.

Considering now the map given by the composition of $\mathbb{R} \times M \rightarrow M \subset T^{*} L$ with the natural projection $\pi: T^{*} L \rightarrow L$, the image of $v_{N}\left(S_{N} \backslash Z_{N}\right)$ provides a cobordism between 
$\gamma=\pi(\tilde{\gamma})$ and $\cup_{i=1}^{l} \gamma_{i}:=\cup_{i=1}^{l} \pi\left(\tilde{\gamma}_{i}\right)$, so $\sum\left[\gamma_{i}\right]=[\gamma]=\beta$. On the other hand, since $v_{N}$ is

$\widehat{J}$-holomorphic, we have

$$
0 \leq \mathcal{A}\left(v_{N}\right):=\int_{S_{N} \backslash Z_{N}} v_{N}^{*} d \alpha=\int_{\partial\left(S_{N} \backslash Z_{N}\right)} v_{N}^{*} \alpha=\int_{\tilde{\gamma}} \alpha-\sum_{i=1}^{l} \int_{\tilde{\gamma}_{i}} \alpha=\ell_{g}(\gamma)-\sum_{i=1}^{l} \ell_{g}\left(\gamma_{i}\right) .
$$

Notice now that, $\bar{S}$ being homeomorphic to a cylinder, the building provides a capping of all but one of the $\tilde{\gamma}_{i}$. Projecting these cappings to $L$, we deduce that only one of the $\gamma_{i}$ is homologically non-trivial. Thus, one of the $\gamma_{i}$ represents the class $\beta$, while all the others vanish in homology. Since $\gamma$ is the unique minimal geodesic in class $\beta$, we infer that $\left\{\gamma_{1}, \ldots, \gamma_{l}\right\}=\{\gamma\}$, that $\mathcal{A}\left(v_{N}\right)=0$, so that $v_{N}$ is a trivial cylinder above the Reeb orbit $\tilde{\gamma}$. Iterating this argument for the floors below, we see that the stable holomorphic building consists only of the holomorphic map $v_{0}: S_{0} \backslash Z_{0} \rightarrow T^{*} L$ with a unique puncture $\left(\# Z_{0}=1\right)$ at which $v_{0}$ is asymptotic to $\tilde{\gamma}$. By point (iii) above, $S_{0}$ is a disc, so

$$
v_{0}:(D \backslash\{0\}, \partial D) \rightarrow\left(T^{*} L, L\right)
$$

is $J$-holomorphic and asymptotic to $\tilde{\gamma}$ at 0 .

\subsubsection{The splitting construction}

In the following we will use a well-known splitting construction, also called stretching the neck in the literature $\left[\mathrm{BEH}^{+} 03\right]$. In this section we review this construction and explain our viewpoint on the different objects introduced by the theory. We insist on our definition of the completion of a symplectic cobordism, which might slightly differ from the usual one.

Symplectic cobordisms. A symplectic cobordism is a compact symplectic manifold $(X, \omega)$ with boundaries $M^{ \pm}$of positive (resp. negative) contact type: in a neighbourhood of $M^{ \pm}, \omega=d \lambda_{ \pm}$is exact, and the corresponding Liouville vector field, defined by $\omega\left(Y_{ \pm}, \cdot\right)=\lambda_{ \pm}$, points outside $X$ for positive boundaries and inside $X$ for negative ones. Then $\alpha_{ \pm}:=\lambda_{ \pm \mid M^{ \pm}}$is a contact form on $M^{ \pm}$. One of the boundaries may be empty but not both. It is called an exact symplectic cobordism if $\omega$ has a global primitive $\lambda$ on $X$ and $\lambda_{ \pm}=\lambda$. This should not be confused with the case where $(X, \omega)$ is an exact symplectic manifold and $\lambda_{ \pm} \neq \lambda$. We illustrate these points using the main examples that will appear in the sequel. Let $(L, g)$ be a closed Riemannian manifold.

- $(\overline{\mathcal{W}(L, g, r)}, d \lambda)$ is an exact symplectic cobordism with $M^{-}=\emptyset, M^{+}=\partial \mathcal{W}(L, g, r)$.

- For $r>r^{\prime}, \overline{\mathcal{W}(L, g, r)} \backslash \mathcal{W}\left(L, g, r^{\prime}\right)$ is an exact symplectic cobordism with $M^{+}=$ $\partial \mathcal{W}(L, g, r)$ and $M^{-}=\partial \mathcal{W}\left(L, g, r^{\prime}\right)$.

- If $L^{\prime} \subset T^{*} L$ is a Lagrangian submanifold and $\mathcal{W}^{\prime} \subset \mathcal{W}(L, g, r)$ is a Weinstein neighbourhood of $L^{\prime}, \overline{\mathcal{W}(L, g, r)} \backslash \mathcal{W}^{\prime}$ is exact as a symplectic manifold since it lies in $T^{*} L$, but it is an exact symplectic cobordism only if $L^{\prime}$ is an exact Lagrangian submanifold of $T^{*} L$. 
Cylindrical ends and completions. In a neighbourhood of a boundary component of a symplectic cobordism (say positive), the transverse Liouville vector field provides a coordinate $r$ and a symplectic identification between this neighbourhood and $\left(M^{+} \times(1-\right.$ $\left.\varepsilon, 1], d\left(r \alpha_{+}\right)\right)$. One can then glue a cylindrical end $M^{+} \times[1, \infty)$ to $X$ and obtain a completion of $X$ along $M^{+}$(glue $M^{-} \times(0,1]$ for a negative component). From a set theoretic point of view this completion is unambiguous: $\widetilde{X}:=X \sqcup M^{+} \times(1, \infty)$. We now want to give details on the structures involved. First we describe the completion from the differential viewpoint more precisely. Let $f^{+}:(1-\varepsilon, 1) \rightarrow(1-\varepsilon, \infty)$ be a diffeomorphism that coincides with the identity near $1-\varepsilon$ (respectively, $f^{-}:(1,1+\varepsilon) \rightarrow(0,1+\varepsilon)$ is the identity near $\left.1+\varepsilon\right)$. We get a bijection $\Phi: X \backslash M^{+} \rightarrow \widetilde{X}$ given by $\Phi=\operatorname{Id}$ outside $M^{+} \times(1-\varepsilon, 1)\left(\right.$ or $\left.M^{-} \times(1,1+\varepsilon)\right)$ and defined in this region by $\Phi(x, r)=\left(x, f^{+}(r)\right)$. Since $f^{+}$is a diffeomorphism, we get a natural structure of smooth manifold on $\widetilde{X}$ which makes $\Phi$ a diffeomorphism. Then $\widetilde{X}$ can be endowed with the symplectic form $\widetilde{\omega}:=\Phi_{*} \omega$, which we do (the symplectic forms which arise this way are all isomorphic). We insist on this last point: a perhaps more common way to define a symplectic form on the completion is to take $d(r \alpha)$ on the ends, which enlarges the symplectic structure. For instance the completion of $(\overline{\mathcal{W}(L, g, 1)}, d \lambda)$ along its boundary in this way is $\left(T^{*} L, d \lambda\right)$ and has infinite volume. We choose a different path here. For us in this paper, the completion is endowed with a symplectic structure which makes $\widetilde{X}$ symplectomorphic to $X \backslash M^{+}$. On the other hand, on $\widetilde{X}$ we now have dilations on the end $M^{+} \times(1, \infty)$ (contractions if we complete a negative end), and we say that an almost complex structure on $\widetilde{X}$ is cylindrical at infinity if it is compatible with $\widetilde{\omega}$ on $\widetilde{X}$ and compatible with $\alpha_{+}$at infinity, in a similar way as already defined for the cotangent bundle: outside a compact set of $\widetilde{X}$ it sends $r \frac{\partial}{\partial r}$ to the Reeb vector field of $\alpha_{+}$and is invariant by the dilation on the positive end (by the contractions on the negative end, respectively). In other terms, the completion $\widetilde{X}$ coincides with $X \backslash M^{+}$from a symplectic point of view, but it has very specific almost complex structures, which will usually be denoted with a tilde. This completion can be made along several boundaries at a time, and by the completion of $X$ we mean its completion along all boundary components. We say that an almost complex structure on $X \backslash M^{+}$is cylindrical at infinity if it coincides with the pull-back of a cylindrical almost complex structure on the completion $\tilde{X}$ of $X$. Similarly, a holomorphic curve in $X \backslash M^{+}$is said to be asymptotic to some Reeb orbit of a boundary component if it is when pushed forward to the completion $\widetilde{X}$. The following example will be useful in the sequel.

Example 3.22. Since the completion of $\overline{\mathcal{W}(L, g, r)}$ is $T^{*} L$ from the almost complex point of view, the statement of theorem 3.2 for almost complex structures $J \in \mathcal{J}_{\text {cyl,g }}^{\infty}$ is equivalent to the existence of punctured holomorphic discs in $\mathcal{W}(L, g, r)$ with boundary on $L$ and asymptotic to the lift of some minimal geodesic in class $\beta$ to $\partial \mathcal{W}(L, g, r)$ for almost complex structures on $\mathcal{W}(L, g, r)$ cylindrical at infinity. We will freely switch from one setting to the other in the following.

Stretching the neck. Let now $X$ be a symplectic cobordism and $M \subset \operatorname{int} X$ be a (separating) closed contact type hypersurface. As previously, a collar neighbourhood of $M$ in int $X$ is symplectomorphic to $(M \times(1-\varepsilon, 1+\varepsilon), d(r \alpha))$. We start with an almost complex 
structure $J$ that is compatible with $\omega$, cylindrical at infinity on int $X$ and compatible with $\alpha$ in our collar neighbourhood. For small $\varepsilon>0$ we define a collection of manifolds $X_{\varepsilon}$ by replacing our collar neighbourhood in int $X$ with a very large collar $M \times(\varepsilon, 1 / \varepsilon)$, by a procedure analogous to the one described in the previous paragraph. We extend $J$ to an almost complex structure $J_{\varepsilon}$ on $X_{\varepsilon}$ by choosing $J_{\varepsilon}$ to be dilation invariant in the collar. There is a diffeomorphism between $X_{\varepsilon}$ and int $X$ and $X_{\varepsilon}$ can be endowed with the pullback symplectic form. Again, $X_{\varepsilon}$ coincides with int $X$ from the symplectic point of view, but the almost complex structures compatible with $\alpha$ in this larger collar are different. In our context, neck stretching consists in letting the parameter $\varepsilon$ go to zero and studying the behaviour of sequences of $J_{\varepsilon}$-holomorphic curves in $X_{\varepsilon}$. The compactness theorem of $\left[\mathrm{BEH}^{+} 03\right]$ related to this splitting can be summarized in an imprecise way as follows:

Theorem (Bourgeois et al.). Let $J_{n}:=J_{\varepsilon_{n}}$ be a sequence of almost complex structures on a symplectic cobordism, cylindrical and fixed at infinity, that stretch the neck of a closed contact type hypersurface $M$. Assume that $M$ splits $X$ into $X_{+} \cup X_{-}$, with $\partial X_{+}=M^{+} \cup M$ and $\partial X_{-}=M^{-} \cup M$. Let $u_{n}: \Sigma \rightarrow X$ be a sequence of $J_{n}$-holomorphic curves which satisfy some energy bound. Then, after extraction, $u_{n}$ converges to a holomorphic building with finite energy: a collection of punctured holomorphic curves in $M^{+} \times(0,+\infty), \tilde{X}_{+}$, $M \times(0, \infty), \widetilde{X}_{-}, M^{-} \times(0, \infty)$, asymptotic at their punctures to Reeb orbits and organized in levels (see the more detailed descriptions in our specific situations below).

Remarks 3.23. - The statement above makes sense, and still holds, when $M=\emptyset$. Then, the sequence $J_{n}$ does not stretch any neck, and the components of the limit building take values in $M^{+} \times(0,+\infty), \widetilde{X}$ and $M^{-} \times(0,+\infty)$. This is exactly the compactness statement of theorem 3.21.

- We do not wish to discuss here the notion of energy of a curve or a holomorphic building in a cobordism. What we need to know for the rest of the paper is as follows and can also be found in [BEH ${ }^{+}$03]: in $T^{*} L$ this notion coincides with the energy that we defined in §3.6.1. The buildings that arise as a limit of curves with finite energy have finite energy. Then any component of such a building has itself finite energy. And finally, punctured holomorphic curves with finite energy are asymptotic in a $\mathcal{C}^{l}$-sense to trivial cylinders over closed Reeb orbits.

Symplectic area of a building in a split manifold. In the setting defined above, there is a natural concept of symplectic area for a holomorphic building $B$ with finite energy in a split manifold, denoted henceforth $\mathcal{A}(B)$, which is very close to the notion of symplectic energy $\left[\mathrm{BEH}^{+} 03\right]$. This is simply the sum of the symplectic areas of the components in the different pieces of the split manifolds, where

- the symplectic area of a component in a completion $\left(\tilde{X}_{+}, \widetilde{X}_{-}\right.$in the previous statement) is computed with the symplectic form. We recall that int $X$ and $\widetilde{X}$ are symplectomorphic. This quantity is a positive number.

- the symplectic area of a component in a cylindrical piece $M \times(0, \infty)$ is computed by integration of $\pi^{*} d \alpha$ (where $\pi: M \times(0, \infty) \rightarrow M$ is the projection). Notice that 
$\pi^{*} d \alpha$ is not a symplectic form on $M \times(0, \infty)$. This is however a non-negative number when evaluated on a holomorphic curve that vanishes if and only if the component is a trivial cylinder over a Reeb orbit.

The name symplectic area is justified by the following result, proved in $\left[\mathrm{BEH}^{+} 03\right.$, Proposition 9.4] in the language of symplectic energy.

Proposition 3.24. Let $u_{n}$ be a sequence of $J_{n}$-holomorphic curves for a sequence of almost complex structures that stretch the neck of a closed contact type hypersurface (possibly empty). Then, the symplectic area of the limit building is the limit of the symplectic area of the curves $u_{n}$.

For a subbuilding $B^{\prime} \subset B$ the symplectic area $\mathcal{A}\left(B^{\prime}\right)$ has an easy geometric interpretation, which we will not use: it simply represents, up to a small error that goes to 0 with $n \rightarrow \infty$, the symplectic area of the restriction of $u_{n}$ to a subset that converges to this subbuilding (see $\left[\mathrm{BEH}^{+} 03\right]$ for the precise definition of this convergence). We will use however the following fact: in an exact setting (to be made precise in the following statement) the symplectic area of a building depends only on its asymptotic Reeb orbits.

Lemma 3.25. Let $(X, \omega)$ be a symplectic cobordism between $\left(M^{+}, \alpha_{+}\right)$and $\left(M^{-}, \alpha_{-}\right)$such that $\omega=d \lambda$ is exact. Let $(M, \alpha)$ be a closed contact type hypersurface that splits $X$ in $X_{+}$and $X_{-}$. Let $B$ be a finite energy holomorphic building with components in the floors $M^{-} \times(0, \infty), M^{+} \times(0, \infty), M \times(0, \infty), \widetilde{X}_{-}$and $\widetilde{X}_{+} \cdot$ We call $\left\{\gamma_{i}^{+}\right\}$and $\left\{\gamma_{i}^{-}\right\}$the positive and negative Reeb orbits to which $B$ is asymptotic at its positive and negative punctures. Then the symplectic area of $B$ is

$$
\mathcal{A}(B)=\sum_{i} \int_{\gamma_{i}^{+}} \lambda-\sum_{j} \int_{\gamma_{j}^{-}} \lambda .
$$

Proof: The proof is almost immediate. The formula is additive with respect to the decomposition of the building in different floors, hence we only need to verify the formula for each floor.

For the components in $\widetilde{X}_{+}$or $\widetilde{X}_{-}$we can apply Stokes' theorem. For a component in $M \times(0, \infty)$ given by $u: \Sigma \backslash Z \rightarrow M \times(0, \infty)$, where $Z:=\left\{z_{i}\right\}$ is a set of punctures of the closed Riemann surface $\Sigma$ and $\Gamma:=\left\{\gamma_{i}^{+}\right\} \cup\left\{\gamma_{j}^{-}\right\}$their corresponding asymptotic closed Reeb orbits, we proceed as follows. Let $f:(0,1) \rightarrow(0, \infty)$ be an increasing diffeomorphism and $F:=\mathrm{Id} \times f: M \times(0,1) \rightarrow M \times(0, \infty)$ the induced diffeomorphism. By the asymptotic properties of the holomorphic buildings with finite energy, the pull-back $F^{*} u: \Sigma \backslash Z \rightarrow$ $M \times(0,1)$ can be considered as a smooth map of the compact surface $\hat{\Sigma}$ obtained by blowing-up the punctures to $M \times[0,1]$. This map is therefore amenable to Stokes theorem. Moreover, $F^{*}\left(\pi^{*} d \alpha\right)=(\pi \circ F)^{*} d \alpha=\pi^{*} d \alpha=\pi^{*} d \lambda$ because $d \alpha_{\mid M}=\omega_{\mid M}=d \lambda_{\mid M}$. Thus,

$$
\begin{aligned}
\int_{\Sigma \backslash Z} u^{*} \pi^{*} d \alpha & =\int_{\operatorname{int} \hat{\Sigma}}\left(F^{*} u\right)^{*} F^{*} \pi^{*} d \alpha=\int_{\operatorname{int} \hat{\Sigma}}\left(F^{*} u\right)^{*} \pi^{*} d \lambda=\int_{\hat{\Sigma}}\left(F^{*} u\right)^{*} \pi^{*} d \lambda=\int_{\partial \hat{\Sigma}}(\pi \circ u)^{*} \lambda \\
& =\sum_{\gamma \in \Gamma} \int_{\gamma} \lambda=\sum_{i} \int_{\gamma_{i}^{+}} \lambda-\sum_{j} \int_{\gamma_{j}^{-}} \lambda .
\end{aligned}
$$




\subsubsection{Compactness for punctured holomorphic disks II}

We recall the setting: $g$ is a Riemannian metric on $L$ and $M=\left\{\|p\|_{g}=1\right\}$. Let $\mathcal{W}^{\prime}$ be a smoothly bounded open neighborhood of the zero section such that $\left(M^{\prime}:=\partial \mathcal{W}^{\prime}, \alpha^{\prime}\right)$ is a closed hypersurface of contact type. Assume that the projection $\pi: T^{*} L \rightarrow L$ induces an isomorphism $\pi_{*}: H_{1}\left(\mathcal{W}^{\prime}\right) \rightarrow H_{1}(L)$. We consider a sequence $J_{n} \in \mathcal{J}_{\text {cyl }, g}^{\infty}$ of almost complex structures which stretch the neck near $M^{\prime}$, in particular these almost complex structures are cylindrical and fixed in the complement of a compact set of $T^{*} L$. For a chosen class $\beta \in H_{1}(L)$ and $\tilde{\gamma}$ a lift of a minimal geodesic $\gamma$ representing $\beta$, let $u_{n}: D \backslash\{0\} \rightarrow T^{*} L$ be a sequence of $J_{n}$-holomorphic punctured disks such that $u_{n}(\partial D) \subset L,\left[u_{n}(\partial D)\right]=\beta$ and $u_{n}$ is asymptotic to $\tilde{\gamma}$ at 0 . We write $\widetilde{T^{*} L \backslash \mathcal{W}^{\prime}}$ for the completion of $T^{*} L \backslash \mathcal{W}^{\prime}$ with a negative cylindrical end $(0,1) \times M^{\prime}$ and $\widetilde{\mathcal{W}^{\prime}}$ for the completion of $\mathcal{W}^{\prime}$ with a positive cylindrical end $(1, \infty) \times M^{\prime}$. Note that these completions are symplectomorphic to the underlying spaces. Recall also that the splitting process provides almost complex structures, each denoted $\widetilde{J}$, on $(0, \infty) \times M, \widetilde{T^{*} L \backslash \mathcal{W}^{\prime}},(0, \infty) \times M^{\prime}$ and $\widetilde{\mathcal{W}^{\prime}}$. These structures are cylindrical at infinity in $\widetilde{T^{*} L \backslash \mathcal{W}^{\prime}}$ and $\widetilde{\mathcal{W}^{\prime}}$ for the contact form $\alpha^{\prime}$, and cylindrical in $(0, \infty) \times M^{\prime}$ (meaning that it is compatible with $\alpha^{\prime}$ and $\mathbb{R}_{*}^{+}$-invariant).

Theorem 3.26. The sequence $\left(u_{n}\right)_{n \in \mathbb{N}}$ converges to a holomorphic building with the following properties:

- it has no component in $(0, \infty) \times M$ (in other terms, the top floor of the holomorphic building is $\left.\widetilde{T^{*} L \backslash \mathcal{W}^{\prime}}\right)$,

- it contains, as a subbuilding, a disc with one positive puncture with boundary on $L$ and asymptotic at the puncture to a Reeb orbit $\tilde{\gamma}^{\prime}$ in $M^{\prime}$ that projects to a representative of $\beta \in H_{1}(L)$,

- if $\widetilde{T^{*} L \backslash \mathcal{W}^{\prime}}$ is exact (i.e. $\alpha^{\prime}=\lambda_{\mid M^{\prime}}$ ) we can be more precise: the bottom level contains a $\widetilde{J}$-holomorphic map $v_{0}:(D \backslash\{0\}, \partial D) \rightarrow\left(\widetilde{\mathcal{W}^{\prime}}, L\right)$ that is asymptotic to a Reeb orbit $\tilde{\gamma}^{\prime}$ in $M^{\prime}$ that projects to a representative of $\left.\beta \in H_{1}(L)\right)$.

Proof: Since all $u_{n}$ are asymptotic to $\tilde{\gamma}$ at 0 , by corollary 3.20 the energy $\mathcal{E}\left(u_{n}\right)$ is uniformly bounded. The SFT compactness theorem for split symplectic manifolds $\left[\mathrm{BEH}^{+} 03, \mathrm{Abb} 14\right]$ implies that our sequence $u_{n}:(D \backslash\{0\}, \partial D, j) \rightarrow\left(T^{*} L, L, J_{n}\right)$ converges to a stable holomorphic building. In our situation this is given by the following data.

(i) $v_{0}:\left(S_{0} \backslash Z_{0}, \partial S_{0}, j_{0}\right) \rightarrow\left(\widetilde{\mathcal{W}^{\prime}}, L, \widetilde{J}\right)$ is a proper $\widetilde{J}$-holomorphic map from a compact Riemann surface $S_{0}$ with boundary $\partial S_{0}$ with a finite set of punctures $Z_{0} \subset S_{0} \backslash \partial S_{0}$ to the almost complex manifold $\left(\widetilde{\mathcal{W}^{\prime}}, \widetilde{J}\right)$ with finite energy. Moreover, although $S_{0}$ may have several components, $\partial S_{0}$ is a unique circle, $v_{0}\left(\partial S_{0}\right) \subset L$, and since $\left(u_{n}\right)_{*}[\partial D]=\beta$, $v_{0}\left(\partial S_{0}\right)$ represents the class $\beta$ in $H_{1}(L)$. The almost complex structure $\widetilde{J}$ is cylindrical at infinity on $\widetilde{\mathcal{W}^{\prime}}$ (for the contact form $\alpha^{\prime}$ ).

(ii) For $k=1, \ldots, p-1$ we have holomorphic maps

$$
v_{k}:\left(S_{k} \backslash Z_{k}, j_{k}\right) \longrightarrow\left((0, \infty) \times M^{\prime}, \widetilde{J}\right)
$$


from closed Riemann surfaces $S_{k}$ with a finite set of punctures $Z_{k} \subset S_{k}$ to the symplectization $(0, \infty) \times M^{\prime}$. The almost complex structure $\widetilde{J}$ is cylindrical with respect to the contact form $\alpha^{\prime}$. As in section 3.6.2, there are decoration maps $\Phi_{k}$ for $k=1, \ldots, p-1$ that glue all of the negative punctures of $Z_{k}$ to the positive punctures of $Z_{k-1}$ in the corresponding oriented blow-ups $\bar{S}_{k}$ and $\bar{S}_{k-1}$.

(iii) $v_{p}:\left(S_{p} \backslash Z_{p}, j_{p}\right) \rightarrow\left(\widetilde{T^{*} L \backslash \mathcal{W}^{\prime}}, \widetilde{J}\right)$ is a $\widetilde{J}$-holomorphic map from a closed Riemann surface $S_{p}$ with a finite set of punctures $Z_{p} \subset S_{p}$ to the almost complex manifold $\left(\widetilde{T^{*} L \backslash \mathcal{W}^{\prime}}, \widetilde{J}\right)$ such that $v_{p}$ has finite energy. The almost complex structure $\widetilde{J}$ is cylindrical at infinity at both ends of $\widetilde{T^{*} L \backslash \mathcal{W}^{\prime}}$. Furthermore, similar to (ii), there is a decoration map $\Phi_{p}$ that glues all of the negative punctures of $Z_{p}$ to the positive punctures of $Z_{p-1}$ in the corresponding oriented blow-ups $\bar{S}_{p}$ and $\bar{S}_{p-1}$.

(iv) For $k=p+1, \ldots, q$ we have holomorphic maps

$$
v_{k}:\left(S_{k} \backslash Z_{k}, j_{k}\right) \longrightarrow((0, \infty) \times M, \widetilde{J})
$$

from closed Riemann surfaces $S_{k}$ with a finite set of punctures $Z_{k} \subset S_{k}$ to the symplectization $(0, \infty) \times M$. As before, $\widetilde{J}$ is cylindrical on $(0, \infty) \times M$ with respect to the contact form $\alpha$. Similar to (ii), there are decoration maps $\Phi_{k}$ for $k=p+1, \ldots, q$ that glue all of the negative punctures of $Z_{k}$ to the positive punctures of $Z_{k-1}$ in the corresponding oriented blow-ups $\bar{S}_{k}$ and $\bar{S}_{k-1}$

(v) Denote by

$$
\bar{S}:=\bar{S}_{0} \cup_{\Phi_{1}} \bar{S}_{1} \cup \ldots \cup_{\Phi_{p}} \bar{S}_{p} \cup_{\Phi_{p+1}} \ldots \cup_{\Phi_{q}} \bar{S}_{q}
$$

the piecewise smooth surface obtained by gluing together all blow ups $\bar{S}_{k}$ at their punctures via the decoration maps $\Phi_{k}$. Then $\bar{S}$ has no nodal point and is homeomorphic to $\widehat{D_{0}}:=\mathbb{R}_{\geq 0} \times S^{1} \cup\{\infty\} \times S^{1}$, which is homeomorphic to the oriented blow-up of $D$ at 0 . Again, this is due to the exactness of the manifold (not to be confused with exactness of the cobordism), which prevents the formation of sphere or disc bubbles by lemma 3.25 .

We now analyze the limit holomorphic building. Notice first that the argument for the top floor in the proof of theorem 3.21 applies word for word and proves the first assertion of our theorem: there is no component of the limit holomorphic building in $(0, \infty) \times M$.

Now by a subbuilding we mean a collection of connected components $\left(S_{i}^{j}\right)$ of the $S_{i}$ together with the maps $v_{i}^{j}:=v_{i \mid S_{i}^{j}}$. We say that a subbuilding is connected if the surface obtained by gluing the $\bar{S}_{i}^{j}$ via the corresponding decoration maps is connected. We focus first on the lowest level of the limit building, i.e. we consider only the map $v_{0}:\left(S_{0} \backslash Z_{0}, \partial S_{0}, j_{0}\right) \rightarrow$ $\left(\widetilde{\mathcal{W}^{\prime}}, L, \widetilde{J}\right)$ and restrict to the component of $S_{0}$ containing the boundary $\partial S_{0}$. We label this component by $S_{0}^{\partial}$ and write $v_{0}^{\partial}$ for the restricted map. Since the domain of our sequence of maps is a punctured disc, the domain $S_{0}^{\partial} \backslash Z_{0}^{\partial}$ can only be a punctured disc, although with arbitrarily many punctures that we call $\left\{z_{1}, \ldots, z_{l}\right\}$. Thus $S_{0}^{\partial} \backslash Z_{0}^{\partial}=D \backslash\left\{z_{1}, \ldots, z_{l}\right\}$ and $v_{0}^{\partial}$ is asymptotic to Reeb orbits $\tilde{\gamma}_{i}^{\prime}$ at the punctures $z_{i}$. For each puncture $z_{i}$, let $B\left(z_{i}\right)$ denote 


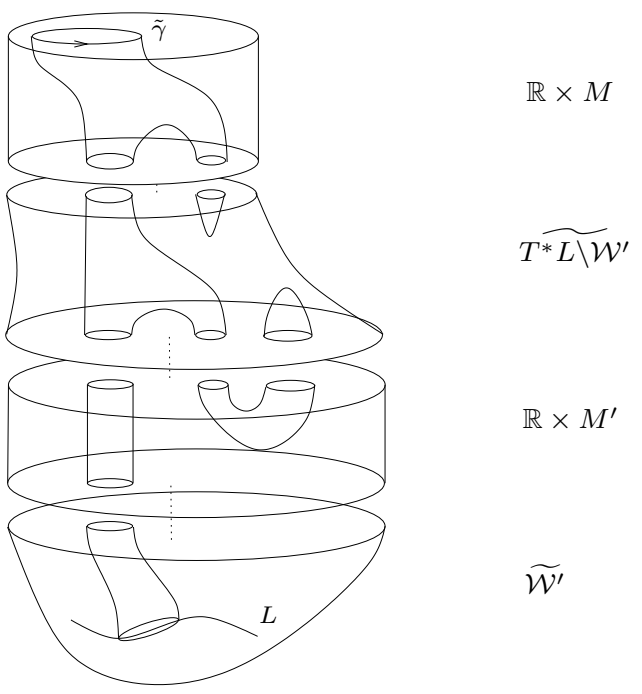

Figure 2: A limit holomorphic building when stretching the neck of $M^{\prime}$

the maximal connected subbuilding which has $\tilde{\gamma}_{i}^{\prime}$ as a single negative puncture. Since $\bar{S}$ is homeomorphic to an annulus, exactly one of the $B\left(z_{i}\right)$, say $B\left(z_{1}\right)$, is a topological annulus, while all the other ones are topological discs. The subbuilding composed of $S_{0}^{\partial}$ and the $B\left(z_{i}\right), i=2, \ldots, n$ is the required subbuilding: it is indeed a topological disc with one positive puncture at $z_{1}$ and with boundary on $L$. Moreover, its projection to $L$ - welldefined because each floor of the decomposition of $T^{*} L$ naturally projects to $L$ - provides a homotopy between $\pi\left({\tilde{\gamma_{1}}}^{\prime}\right)$ and $v_{0}\left(\partial S_{0}\right)$, which represents the class $\beta$ in $H_{1}(L)$.

Finally, when $\widetilde{T^{*} L \backslash \mathcal{W}^{\prime}}$ is an exact cobordism we show that the $B\left(z_{i}\right), i \geq 2$, simply do not appear. Indeed, for each $i \geq 2, B\left(z_{i}\right)$ has a maximal floor, whose components therein have only negative punctures. By the maximal principle these maximal floors cannot lie in $(0, \infty) \times M$ nor $(0, \infty) \times M^{\prime}$, so they have to be contained in $\widetilde{T^{*} L \backslash \mathcal{W}^{\prime}}$. Now $\widetilde{T * L \backslash \mathcal{W}^{\prime}}$ is an exact cobordism by assumption and the almost complex structure $\widetilde{J}$ tames the exact symplectic form. By Stokes' Theorem components with only negative punctures do not exist in $\widetilde{T^{*} L \backslash \mathcal{W}^{\prime}}$, since they would have non-positive symplectic area. Hence we may rule out the existence of such holomorphic subbuildings and we see that $v_{0}^{\partial}$ has only one (positive) puncture, whose asymptotic we call $\tilde{\gamma}^{\prime} \subset M^{\prime}$. Proving that $\left[\tilde{\gamma}^{\prime}\right]=\beta$ is similar to the non-exact case above.

\subsection{Proof of theorem 3.2}

Let $g$ be a Riemannian metric on $L$ satisfying the assumptions of theorem 3.2 and let $\varepsilon>0$. We divide the proof into two steps. We first prove our theorem for the Riemannian metric $g^{\prime}:=g_{\varepsilon, \beta}$ provided by proposition 3.15 . In particular it is $\varepsilon$-close to $g$ in the $\mathcal{C}^{0}$-norm. We consider the metric $g$ itself in the second step. 
Step 1: proof of theorem 3.2 for those $J$ that coincide with $J_{g^{\prime}}$ at infinity. Let $J_{g^{\prime}} \in \mathcal{J}_{\mathrm{Cyl}, g^{\prime}}^{\infty}$ be the almost complex structure as defined in section 3.1. Let $\mathcal{J}_{g^{\prime}}$ denote in this paragraph the set of almost complex structures on $T^{*} L$ that coincide with $J_{g^{\prime}}$ outside a compact subset. We first show that the set $\mathcal{M}(J, \beta)$ (defined on p. 8) contains at least one element for all $J \in \mathcal{J}_{g^{\prime}}$. Recall that by construction $g^{\prime}$ has a unique minimizing geodesic $\gamma(\beta)$ of the form $\gamma\left(\beta^{\prime}\right)^{k}$ for some primitive geodesic $\gamma\left(\beta^{\prime}\right)$, where $k \geq 1$ and $\beta=k \beta^{\prime}$. Lemma 3.17 then implies that the space $\mathcal{M}\left(J, \beta^{\prime}\right)$ and its subspace consisting of somewhere injective curves coincide for all $J \in \mathcal{J}_{\mathrm{Cyl}, g^{\prime}}^{\infty}$.

The proof relies on the compactness theorem 3.21 and the Fredholm theory for punctured holomorphic discs with boundary on a Lagrangian as summarized in the following lemma. Although it may be considered folklore we explain this lemma in appendix A.

Lemma 3.27. There is a subset $\mathcal{J}_{g^{\prime}}^{\text {Reg }} \subset \mathcal{J}_{g^{\prime}}$, dense in the $\mathcal{C}^{\infty}$-topology, such that for all $J \in \mathcal{J}_{g^{\prime}}^{\text {Reg }}$ the set $\mathcal{M}\left(J, \beta^{\prime}\right)$ is a manifold of dimension 0 . Moreover, if $J_{0}, J_{1} \in \mathcal{J}_{g^{\prime}}^{\text {Reg }}$, for a generic smooth path $\left\{J_{t}\right\}_{t \in[0,1]}$ in $\mathcal{J}_{g^{\prime}}$ that interpolates between $J_{0}$ and $J_{1}$, the space $\cup_{t \in[0,1]} \mathcal{M}\left(J_{t}, \beta^{\prime}\right)$ is a smooth manifold of dimension 1 .

Proposition 3.15 shows that $\mathcal{M}\left(J_{g^{\prime}}, \beta^{\prime}\right)$ consists of exactly one element and $J_{g^{\prime}} \in \mathcal{J}_{g^{\prime}}^{\text {Reg }}$. Let $J \in \mathcal{J}_{g^{\prime}}^{\text {Reg }}$ and $\left\{J_{t}\right\}$ be a regular path between $J_{g^{\prime}}$ and $J$ consisting of almost complex structures which coincide with $J_{g^{\prime}}$ outside a compact set, as defined in lemma 3.27. Then $\cup_{t \in[0,1]} \mathcal{M}\left(J_{t}, \beta^{\prime}\right)$ is a one-dimensional cobordism between $\mathcal{M}\left(J_{g^{\prime}}, \beta^{\prime}\right)$ and $\mathcal{M}\left(J, \beta^{\prime}\right)$ by lemma 3.27 and is compact by theorem 3.21. Both ends of the cobordism therefore have the same - odd - parity, so $\mathcal{M}\left(J, \beta^{\prime}\right)$ is non-empty when $J$ is regular. Finally, if $J \in \mathcal{J}_{g^{\prime}}$ does not belong to $\mathcal{J}_{g^{\prime}}^{\text {Reg }}$, let $J_{n} \in \mathcal{J}_{g^{\prime}}^{\text {Reg }}$ be a sequence of regular almost complex structures that converge to $J$ in the $\mathcal{C}^{\infty}$-topology. The previous argument shows that there exist elements $u_{n} \in \mathcal{M}\left(J_{n}, \beta^{\prime}\right)$ for all $n$. By theorem 3.21 we can extract from $u_{n}$ a subsequence that converges to an element $u \in \mathcal{M}\left(J, \beta^{\prime}\right)$, thus $\mathcal{M}\left(J, \beta^{\prime}\right)$ is non-empty. Finally, if $u \in \mathcal{M}\left(J, \beta^{\prime}\right)$, its $k$-cover $u\left(z^{k}\right) \in \mathcal{M}(J, \beta)$, so $\mathcal{M}(J, \beta)$ itself is indeed non-empty. This completes the first step of the proof.

Before we prove theorem 3.2 for $g$ itself, let us recall that by example 3.22, establishing the existence of a punctured holomorphic disc asymptotic to a lift of a geodesic $\gamma$ at the puncture for all structures in $\mathcal{J}_{\mathrm{Cyl}, h}^{\infty}\left(T^{*} L\right)$ is equivalent to establishing their existence for all elements of $\mathcal{J}_{\mathrm{Cyl}, h}^{\infty}(\mathcal{W}(L, h, r)$ ) (the almost complex structures that are cylindrical at infinity in $\mathcal{W}(L, h, r))$. We will freely switch from one problem to the other in the remaining of this paper. In particular the previous theorems (compactness, neck stretching) are applicable in this new setting.

Step 2: from $g^{\prime}$ to $g$. Let now $J \in \mathcal{J}_{\mathrm{Cyl}, g}^{\infty}(\mathcal{W}(L, g, 1))$ Since $g^{\prime}$ is $\varepsilon$-close to $g$ in the $\mathcal{C}^{0}$-topology, $\mathcal{W}\left(L, g^{\prime},(1-\varepsilon)^{-1 / 2}\right):=\left\{\|p\|_{g^{\prime}}<(1-\varepsilon)^{-1 / 2}\right\} \ni \mathcal{W}(L, g, 1)$. We can therefore consider a sequence of almost complex structures $J_{n}$ that are cylindrical at infinity in $\mathcal{W}\left(L, g^{\prime},(1-\varepsilon)^{-1 / 2}\right)$, stretch the neck along $\partial \mathcal{W}(L, g, 1)$, and converge to $J$ in $\mathcal{C}_{\text {loc }}^{\infty}$ on $\mathcal{W}(L, g, 1)$. By the discussion above, the first step of our proof guarantees that for every $n$, 
there exists a punctured $J_{n}$-holomorphic disc $u_{n}:(D \backslash\{0\}, \partial D) \rightarrow\left(\mathcal{W}\left(L, g^{\prime},(1-\varepsilon)^{-1 / 2}\right), L\right)$ asymptotic to $\tilde{\gamma}^{\prime}$ (the lift of the unique $g^{\prime}$-minimizing curve in class $\beta$ ). Applying theorem 3.26 to the sequence of punctured holomorphic discs $u_{n}$, we get a limit holomorphic building $\left(v_{0}, \ldots, v_{p}, \ldots, v_{q}\right)$ as described in the section 3.6.4. Since we have an exact symplectic cobordism, the domain of $v_{0}$ contains a punctured disc $D \backslash\{0\}$ and $v_{0}^{\partial}:=v_{0 \mid D \backslash\{0\}}$ is asymptotic at the puncture to a Reeb orbit $\tilde{\gamma} \subset \partial \mathcal{W}(L, g, 1)$, whose projection to $L$ is a geodesic $\gamma$ that represents the class $\beta$. Gluing all the components of the building together, except for $\left(v_{0}^{\partial}, D \backslash\{0\}\right)$, we get a subbuilding which is a punctured 2-sphere with one positive puncture asymptotic to $\tilde{\gamma}^{\prime}$ and one negative puncture asymptotic to $\tilde{\gamma}$. By lemma 3.25 (we are indeed in the exact setting), the symplectic area of this subbuilding is therefore

$$
0<(1-\varepsilon)^{-1 / 2} \ell_{g^{\prime}}\left(\gamma^{\prime}\right)-\ell_{g}(\gamma)
$$

so $\ell_{g}(\gamma)<(1-\varepsilon)^{-1 / 2} \ell_{g^{\prime}}\left(\gamma^{\prime}\right)$. On the other hand, since $g^{\prime}$ is $\varepsilon$-close to $g$ in the $\mathcal{C}^{0}$-topology, we get

$$
\ell_{g}^{\min }(\beta) \leq \ell_{g}(\gamma)<\frac{1}{(1-\varepsilon)^{1 / 2}} \ell_{g^{\prime}}^{\min }(\beta) \leq \sqrt{\frac{1+\varepsilon}{1-\varepsilon}} \ell_{g}^{\min }(\beta) .
$$

Since $g$ has a discrete length spectrum in the class $\beta$, we can take $\varepsilon$ much smaller than the gap between the length of the $\beta$-minimizing $g$-geodesic and the other elements in this spectrum. In view of the estimation above, we see that $\gamma$ must then be a $\beta$-minimizing geodesic.

Notice also that, as stated in remark 3.3, if $g$ does not have a discrete length spectrum in class $\beta$, the proof above shows the existence of a punctured holomorphic disc with boundary on $L$, now not necessarily asymptotic to the minimal geodesic in class $\beta$, but still asymptotic to a geodesic in class $\beta$ with length $\varepsilon$-close to the minimal length.

\section{$4 \mathcal{C}^{0}$-rigidity of the area homomorphism and the Maslov class}

\subsection{The Maslov class of a Lagrangian}

We define the Maslov index $\mu_{L}^{\tau}$ of a Lagrangian submanifold $L$ and state some useful properties. We refer to [MS12, Appendix C.3] for a general treatment of the Maslov index. First recall that given any two Lagrangian distributions $\tau, \tau^{\prime}$ along a loop $\gamma: S^{1} \rightarrow(M, \omega)$, the relative Maslov index

$$
\mu_{\gamma}\left(\tau, \tau^{\prime}\right) \in \mathbb{Z}
$$

is a homotopy invariant that counts (with signs and multiplicities) the number of nontransverse intersections of $\tau$ and $\tau^{\prime}$ along $\gamma$. Furthermore, one can show that for three Lagrangian distributions $\tau, \tau^{\prime}, \tau^{\prime \prime}$ we have the composition equality

$$
\mu_{\gamma}\left(\tau, \tau^{\prime \prime}\right)=\mu_{\gamma}\left(\tau, \tau^{\prime}\right)+\mu_{\gamma}\left(\tau^{\prime}, \tau^{\prime \prime}\right)
$$

Let then $u:(D, \partial D) \longrightarrow(M, L)$ be a disc with boundary on a Lagrangian submanifold $L \subset$ $M, \gamma:=u_{\mid \partial D}$, and let $\tau$ be a Lagrangian distribution of $u^{*} T M$. Then $\mu_{L}^{\tau}(u):=\mu_{\gamma}(T L, \tau)$ is 
the Maslov index of the loop of Lagrangian subspaces $T_{\gamma} L$ relative to $\tau_{\mid \gamma}$. This index does not depend on the choice of $\tau$ (recall that $\tau$ is a Lagrangian distribution over the whole disc $D)$ nor the representative of $[u]$ in $\pi_{2}(M, L)$. We therefore get a map $\mu_{L}: \pi_{2}(M, L) \rightarrow \mathbb{Z}$ that assigns to each class this index. Note that to emphasize the ambient manifold we may sometimes write $\mu_{L}^{\tau}([u], M)$. Given the above formula, if $\tau^{\prime}$ denotes any Lagrangian distribution along $\gamma:=u_{\mid \partial D}$, we have

$$
\mu_{L}^{\tau}([u])=\mu_{\gamma}(T L, \tau)=\mu_{\gamma}\left(T L, \tau^{\prime}\right)+\mu_{\gamma}\left(\tau^{\prime}, \tau\right)
$$

When a symplectic manifold has a globally defined Lagrangian distribution $\tau$ - e.g. cotangent bundles with their vertical distributions - we can define a map $\mu_{L}^{\tau}: H_{1}(L) \rightarrow \mathbb{Z}$ by simply assigning to each class $\beta \in H_{1}(L)$ the Maslov index of the loop $t \mapsto T_{\gamma(t)} L$ relative to $\tau$ for an arbitrary choice of representative $\gamma$ of $\beta$, i.e. $\mu_{L}^{\tau}(\beta)=\mu_{\gamma}(T L, \tau)$. Notice that, in this case, the Maslov class depends on the choice of $\tau$.

\subsection{Area homomorphisms and Maslov classes of closeby Lagrangian sub- manifolds}

We now aim at explaining the proofs of theorems 1 and 3 . We recall the context. Given a closed Riemannian manifold $(L, g)$ and a class $\beta \in H_{1}(L)$, we denote by $\ell_{g}^{\min }(\beta)$ the minimal length of loops that represent the class $\beta$. The Riemannian structure endows the zero section of the cotangent bundle with a basis of neighbourhoods denoted $\mathcal{W}(L, g, \varepsilon)$. By the Weinstein neighbourhood theorem, whenever $L$ has a Lagrangian embedding into a symplectic manifold $M$, this embedding can be extended to a symplectic embedding of $\mathcal{W}(L, g, \varepsilon)$, that furthermore transports the vertical distribution of $T^{*} L$ to a Lagrangian distribution that we call $\tau$ in the sequel. We simply denote such data by $L \subset \mathcal{W}(L, g, \varepsilon) \subset$ $M$. We recall theorem 3 and then prove theorem 1 .

Theorem 3. Let $(L, g)$ and $\left(L^{\prime}, g^{\prime}\right)$ be two closed Riemannian manifolds and $\iota: L^{\prime} \hookrightarrow$ $\left(T^{*} L, d \lambda\right)$ a Lagrangian embedding such that $\pi_{*} \circ \iota_{*}: H_{1}\left(L^{\prime}\right) \rightarrow H_{1}(L)$ is an isomorphism. Assume that $\iota$ extends to a symplectic embedding $\mathcal{I}$ of a neighborhood of $L^{\prime}$ such that

$$
L \subset \mathcal{I}\left(\mathcal{W}\left(L^{\prime}, g^{\prime}, r^{\prime}\right)\right) \subset \mathcal{W}(L, g, r) \subset T^{*} L
$$

for some $r, r^{\prime}>0$. Then for all $\beta^{\prime} \in H_{1}\left(L^{\prime} ; \mathbb{Z}\right)$ we have

a) $\left|\iota^{*} \lambda\left(\beta^{\prime}\right)\right| \leq r \ell_{g}^{\min }\left(\pi_{*} \circ \iota_{*} \beta^{\prime}\right)$,

b) $\mu_{\iota\left(L^{\prime}\right)}^{\tau}\left(\iota_{*} \beta^{\prime}\right)=0$.

Proof of theorem 1: Let $h:(M, \omega) \rightarrow\left(M^{\prime}, \omega^{\prime}\right)$ be a symplectic homeomorphism that takes a closed Lagrangian submanifold $L \subset M$ to a smooth, hence Lagrangian, submanifold $L^{\prime} \subset$ $M^{\prime}$. Let $g, g^{\prime}$ be Riemannian metrics on $L, L^{\prime}$, respectively. By the Weinstein neighbourhood theorem there exists $\varepsilon_{0}, \varepsilon_{0}^{\prime}$ such that $\mathcal{W}\left(L, g, \varepsilon_{0}\right) \subset M$ and $\mathcal{W}\left(L^{\prime}, g^{\prime}, \varepsilon_{0}^{\prime}\right) \subset M^{\prime}$ and we can even assume (by decreasing $\varepsilon_{0}$ if necessary) that $h\left(\mathcal{W}\left(L, g, \varepsilon_{0}\right)\right) \Subset \mathcal{W}\left(L^{\prime}, g^{\prime}, \varepsilon_{0}^{\prime}\right)$. Fix 
$\varepsilon<\varepsilon_{0}$ and choose a sequence of symplectic diffeomorphisms $f_{n}$ that approximate $h$. For $n$ sufficiently large we have

$$
f_{n}(\mathcal{W}(L, g, \varepsilon)) \Subset \mathcal{W}\left(L^{\prime}, g^{\prime}, \varepsilon_{0}^{\prime}\right) .
$$

Since moreover $h(L)=L^{\prime}$, there exists $0<\varepsilon^{\prime \prime}<\varepsilon^{\prime} \leq \varepsilon_{0}^{\prime}$ such that $\mathcal{W}\left(L^{\prime}, g^{\prime}, \varepsilon^{\prime \prime}\right) \Subset$ $h(\mathcal{W}(L, g, \varepsilon)) \Subset \mathcal{W}\left(L^{\prime}, g^{\prime}, \varepsilon^{\prime}\right)$ and thus $\mathcal{W}\left(L^{\prime}, g^{\prime}, \varepsilon^{\prime \prime}\right) \Subset f_{n}(\mathcal{W}(L, g, \varepsilon)) \Subset \mathcal{W}\left(L^{\prime}, g^{\prime}, \varepsilon^{\prime}\right)$ for all $n$ large enough. Since $\{\mathcal{W}(L, g, \varepsilon)\}_{\varepsilon>0}$ is a basis of neighbourhoods of $L, \varepsilon^{\prime}$ can be chosen to tend to 0 when $\varepsilon$ goes to 0 . Putting all this together we therefore get

$$
L^{\prime} \subset f_{n}(\mathcal{W}(L, g, \varepsilon)) \Subset \mathcal{W}\left(L^{\prime}, g^{\prime}, \varepsilon^{\prime}\right)
$$

for all $n$ sufficiently large.

We now prove theorem 1.a). Let $\sigma \in H_{2}(M, L ; \mathbb{Z})$ be represented by a smooth surface $\Sigma$ with boundary on $L$ and $\beta:=\partial \sigma \in H_{1}(L ; \mathbb{Z})$ be represented by $\partial \Sigma$. Then $f_{n}(\Sigma)$ is a smooth surface with boundary on $f_{n}(L)$ and

$$
\mathcal{A}_{\omega^{\prime}}\left(f_{n}(\Sigma)\right)=\mathcal{A}_{\omega}(\Sigma)=\mathcal{A}_{\omega}^{L}(\sigma),
$$

because $f_{n}$ is a symplectic diffeomorphism. Moreover, since $f_{n}(L) \subset \mathcal{W}\left(L^{\prime}, g^{\prime}, \varepsilon^{\prime}\right) \subset\left(T^{*} L^{\prime}, d \lambda^{\prime}\right)$, for $n$ large enough we have

$$
\mathcal{A}_{\omega^{\prime}}^{L^{\prime}}\left(h_{*} \sigma\right)=\mathcal{A}_{\omega^{\prime}}\left(f_{n}(\Sigma)\right)+f_{n}^{*} \lambda^{\prime}(\partial \sigma) .
$$

This can be seen as follows: since $f_{n} \rightarrow h$ in the $\mathcal{C}^{0}$-norm, for $\pi^{\prime}: T^{*} L^{\prime} \rightarrow L^{\prime}$ we see that $\pi^{\prime} \circ f_{n \mid L}: L \rightarrow L^{\prime}$ is $\mathcal{C}^{0}$-close to $h_{\mid L}$. So $\pi^{\prime} \circ f_{n \mid L}$ induces an isomorphism in homology and $\pi_{*}^{\prime} \circ f_{n *}=h_{*}: H_{1}(L) \rightarrow H_{1}\left(L^{\prime}\right)$. Then we can extend $f_{n}(\Sigma)$ by a straight cylinder (in the coordinates provided by $\left.\mathcal{W}\left(L^{\prime}, g^{\prime}, \varepsilon_{0}^{\prime}\right) \supset f_{n}(L)\right)$ that connects $f_{n}(\partial \Sigma) \subset f_{n}(L)$ to $\pi^{\prime}\left(f_{n}(\partial \Sigma)\right) \subset L^{\prime}$ (see [BO16, Lemma 5.1] for the details). The $\omega^{\prime}$-area of this cylinder is given by $f_{n}^{*} \lambda^{\prime}(\partial \sigma)$. Rewriting we thus obtain

$$
\mathcal{A}_{\omega^{\prime}}^{L^{\prime}}\left(h_{*} \sigma\right)=\mathcal{A}_{\omega}^{L}(\sigma)+f_{n}^{*} \lambda^{\prime}(\beta) .
$$

By theorem 3.a),

$$
\left|f_{n}^{*} \lambda^{\prime}(\beta)\right| \leq \varepsilon^{\prime} \ell_{g^{\prime}}^{\min }\left(\pi_{*}^{\prime} \circ f_{n *} \beta\right)
$$

and therefore

$$
\left|\mathcal{A}_{\omega}^{L}(\sigma)-\mathcal{A}_{\omega^{\prime}}^{L^{\prime}}\left(h_{*} \sigma\right)\right| \leq \varepsilon^{\prime} \ell_{g^{\prime}}^{\min }\left(h_{*} \beta\right) .
$$

Since this holds for all $\varepsilon^{\prime}$ sufficiently small, in the limit we get $\mathcal{A}_{\omega}^{L}(\sigma)=\mathcal{A}_{\omega^{\prime}}^{L^{\prime}}\left(h_{*} \sigma\right)$.

We prove theorem 1.b). Let $u:(D, \partial D) \rightarrow(M, L)$ represent a class in $\pi_{2}(M, L), \gamma:=$ $u_{\mid \partial D}, \beta:=[\gamma] \in H_{1}(L)$, and let $\tau$ be a Lagrangian distribution along $u$. We recall that we have fixed a Weinstein neighbourhood $\mathcal{W}^{\prime}$ of $L^{\prime}$, equipped with its projection $\pi^{\prime}: \mathcal{W}^{\prime} \rightarrow L^{\prime}$, and $n$ is large enough so that $f_{n}(L) \subset \mathcal{W}^{\prime}$ and $\pi^{\prime} \circ f_{n \mid L}$ is homotopic to $h_{\mid L}$. We denote by $\tau^{\prime}$ the vertical Lagrangian distribution in $\mathcal{W}^{\prime}$. Let $u_{n}^{\prime}:=f_{n} \circ u, \gamma_{n}^{\prime}:=f_{n} \circ \gamma$ and $\tau_{n}^{\prime}:=f_{n *} \tau$. Since $f_{n}$ is symplectic, $\tau_{n}^{\prime}$ is a Lagrangian distribution of $T M^{\prime}$ along $u_{n}^{\prime}$. Since $\operatorname{Im} \gamma_{n}^{\prime} \subset \mathcal{W}^{\prime}$, the map

$$
\begin{aligned}
\varphi: S^{1} \times[0,1] & \longrightarrow \mathcal{W}^{\prime} \\
(t, r) & \longmapsto r \gamma_{n}^{\prime}(t)
\end{aligned}
$$


provides a cylinder that can be glued to $u_{n}^{\prime}$ in order to produce a disc $u^{\prime}:(D, \partial D) \rightarrow$ $\left(M^{\prime}, L^{\prime}\right)$. We denote $\gamma^{\prime}:=u_{\mid \partial D}^{\prime}$. Since $f_{n}$ is close enough to $h$, this disc represents the class $\left[u^{\prime}\right]=[h \circ u] \in \pi_{2}\left(M^{\prime}, L^{\prime}\right)$. Now $\tau_{n}^{\prime}$ can be continuously extended to a Lagrangian distribution $\tau^{\prime \prime}$ over $u^{\prime}$, with the (automatic) property that $\mu_{\varphi(., r)}\left(\tau^{\prime \prime}, \tau^{\prime}\right)$ does not depend on $r$. As a result, using (15) we have

$$
\begin{aligned}
\mu_{L^{\prime}}\left([h \circ u], M^{\prime}\right) & =\mu_{L^{\prime}}\left(\left[u^{\prime}\right], M^{\prime}\right)=\mu_{\gamma^{\prime}}\left(T L^{\prime}, \tau^{\prime \prime}\right) \\
& =\mu_{\gamma^{\prime}}\left(T L^{\prime}, \tau^{\prime}\right)+\mu_{\gamma^{\prime}}\left(\tau^{\prime}, \tau^{\prime \prime}\right)=\mu_{\gamma^{\prime}}\left(\tau^{\prime}, \tau^{\prime \prime}\right)=\mu_{\gamma_{n}^{\prime}}\left(\tau^{\prime}, \tau^{\prime \prime}\right)=\mu_{\gamma_{n}^{\prime}}\left(\tau^{\prime}, \tau_{n}^{\prime}\right),
\end{aligned}
$$

where $\mu_{\gamma^{\prime}}\left(T L^{\prime}, \tau^{\prime}\right)=0$ because $\tau^{\prime}$ is the vertical fiber distribution in $\mathcal{W}^{\prime}$. Using formula (15) again, we thus have

$$
\mu_{L^{\prime}}\left([h \circ u], M^{\prime}\right)=\mu_{\gamma_{n}^{\prime}}\left(\tau^{\prime}, T f_{n}(L)\right)+\mu_{\gamma_{n}^{\prime}}\left(T f_{n}(L), \tau_{n}^{\prime}\right)=-\mu_{f_{n}(L)}^{\tau^{\prime}}\left(\left[\gamma_{n}^{\prime}\right]\right)+\mu_{f_{n}(L)}^{\tau_{n}^{\prime}}\left(\left[u_{n}^{\prime}\right], M^{\prime}\right) .
$$

By theorem 3.b), $\mu_{f_{n}(L)}^{\tau^{\prime}}\left(\left[\gamma_{n}^{\prime}\right]\right)=0$, and $\mu_{f_{n}(L)}^{\tau_{n}^{\prime}}\left(\left[u_{n}^{\prime}\right], M^{\prime}\right)=\mu_{L}^{\tau}([u], M)$ because $f_{n}$ is symplectic.

\subsection{Proof of theorem 3.a)}

Let $\iota: L^{\prime} \hookrightarrow T^{*} L$ be a Lagrangian embedding which extends to a symplectic embedding $\mathcal{I}: \mathcal{W}\left(L^{\prime}, g^{\prime}, r^{\prime}\right) \hookrightarrow T^{*} L$ such that

$$
L, \iota\left(L^{\prime}\right) \subset \mathcal{I}\left(\mathcal{W}\left(L^{\prime}, g^{\prime}, r^{\prime}\right)\right) \subset \mathcal{W}(L, g, r) \subset T^{*} L .
$$

We fix $\beta^{\prime} \in H_{1}\left(L^{\prime} ; \mathbb{Z}\right)$ and write $\beta:=\pi_{*} \circ \iota_{*} \beta^{\prime}$. We aim at proving that $\left|\iota^{*} \lambda\left(\beta^{\prime}\right)\right| \leq$ $r \ell_{g}^{\min }(\beta)$. We can obviously slightly perturb the metric $g$ to prove the theorem, so we assume henceforth that $g$ satisfies the assumptions of proposition 3.15. In particular, there is a unique minimizing geodesic $\gamma$ in the class $\beta$ and theorem 3.2 applies. Notice that $\iota^{*} \lambda\left(\beta^{\prime}\right)$ is the symplectic area of the cylinder

$$
\begin{aligned}
C_{\iota \circ \gamma^{\prime}}:[0,1] \times \mathbb{R} / \ell \mathbb{Z} & \longrightarrow \mathcal{W}(L, g, r) \\
(s, t) & \longmapsto s \iota \circ \gamma^{\prime}(t),
\end{aligned}
$$

where $\gamma^{\prime}: \mathbb{R} / \ell \mathbb{Z} \rightarrow L^{\prime}$ is any smooth curve that represents the class $\beta^{\prime}$.

Let $J_{n}$ be a sequence of almost complex structures on $\mathcal{W}(L, g, r)$ that are cylindrical at infinity (near $\partial \mathcal{W}(L, g, r)$ ), and that stretch the neck along the contact type hypersurface $\mathcal{I}\left(\partial \mathcal{W}\left(L^{\prime}, g^{\prime}, r^{\prime}\right)\right) \subset \mathcal{W}(L, g, r)$. By theorem 3.2 there exists for each $n$ a $J_{n}$-holomorphic map

$$
u_{n}:(D \backslash\{0\}, \partial D) \longrightarrow(\mathcal{W}(L, g, r), L)
$$

which is asymptotic to the lift $\tilde{\gamma}$ of the minimal geodesic $\gamma$ at 0 . An easy computation shows that the symplectic area $\mathcal{A}\left(u_{n}\right)=r \ell_{g}^{\min }(\beta)$. By theorem 3.26 a subsequence of $\left(u_{n}\right)$ converges to a holomorphic building that contains, as a subbuilding $B$, a punctured disc with boundary on $L$ that represents the class $\beta$ and one positive puncture asymptotic to $\mathcal{I}\left(\tilde{\gamma}^{\prime}\right)$ in $\mathcal{I}\left(\partial \mathcal{W}\left(L^{\prime}, g^{\prime}, r^{\prime}\right)\right)$, the lift of a geodesic $\gamma^{\prime}: \mathbb{R} / \ell^{\prime} \mathbb{Z} \rightarrow L^{\prime}$ that represents the class $\beta^{\prime}$. By 
proposition 3.24 the total symplectic area of the building is $r \ell_{g}^{\min }(\beta)$ and by non-negativity of the area of each subbuilding we get $\mathcal{A}(B) \leq r \ell_{g}^{\min }(\beta)$.

Now $\mathcal{W}(L, g, r)$ is an exact symplectic manifold with $L$ as an exact Lagrangian submanifold, so by lemma 3.25 we can compute the area of $B$ by means of any piecewise smooth cylinder that connects $\mathcal{I}\left(\tilde{\gamma}^{\prime}\right)$ to a curve on $L$ in the class $\beta$ in $\mathcal{W}(L, g, r)$. Such a cylinder can be obtained by concatenation of two cylinders: one is given by

$$
\begin{aligned}
\mathcal{I} \circ C_{\tilde{\gamma}^{\prime}}:[0,1] \times \mathbb{R} / \ell^{\prime} \mathbb{Z} & \longrightarrow \mathcal{W}(L, g, r) \\
(s, t) & \longmapsto \mathcal{I}\left(s \tilde{\gamma}^{\prime}(t)\right),
\end{aligned}
$$

and the other one by $C_{\iota \circ \gamma^{\prime}}$. Notice that $\mathcal{A}\left(\mathcal{I} \circ C_{\tilde{\gamma}^{\prime}}\right) \geq 0$ because it is the image by the symplectomorphism $\mathcal{I}$ of a trivial cylinder on a Reeb orbit of $\partial \mathcal{W}\left(L^{\prime}, g^{\prime}, r^{\prime}\right)$. The area of the second cylinder is $\iota^{*} \lambda\left(\left[\gamma^{\prime}\right]\right)=\iota^{*} \lambda\left(\beta^{\prime}\right)$, as already noticed.

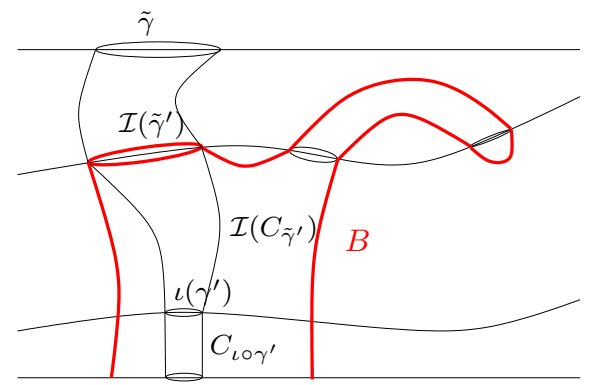

Figure 3: Estimating $\left|\iota^{*} \lambda\left(\left[\gamma^{\prime}\right]\right)\right|: r \ell_{g}(\gamma)>\mathcal{A}(B)=\mathcal{A}\left(\mathcal{I}\left(C_{\tilde{\gamma}^{\prime}}\right)\right)+\mathcal{A}\left(C_{\iota \circ \gamma^{\prime}}\right) \geq 0+\iota^{*} \lambda\left(\left[\gamma^{\prime}\right]\right)$.

We therefore obtain

$$
r \ell_{g}^{\min }(\beta) \geq \mathcal{A}(B)=\mathcal{A}\left(\mathcal{I} \circ C_{\tilde{\gamma}^{\prime}}\right)+\iota^{*} \lambda\left(\beta^{\prime}\right) \geq \iota^{*} \lambda\left(\beta^{\prime}\right)
$$

Considering the class $-\beta^{\prime}$ instead of $\beta^{\prime}$ in the last inequality, we have $\ell_{g}^{\min }(-\beta)=\ell_{g}^{\min }(\beta)$, thus only the sign of $\iota^{*} \lambda\left(\beta^{\prime}\right)$ changes and we get

$$
\left|\iota^{*} \lambda\left(\beta^{\prime}\right)\right| \leq r \ell_{g}^{\min }\left(\pi_{*} \circ \iota_{*} \beta^{\prime}\right) .
$$

\subsection{Proof of theorem 3.b)}

We recall the assumptions of the theorem: $\iota: L^{\prime} \hookrightarrow T^{*} L$ is a Lagrangian embedding which extends to a symplectic embedding $\mathcal{I}: \mathcal{W}\left(L^{\prime}, g^{\prime}, r^{\prime}\right) \hookrightarrow T^{*} L$ such that

$$
L, \iota\left(L^{\prime}\right) \subset \mathcal{W}^{\prime}:=\mathcal{I}\left(\mathcal{W}\left(L^{\prime}, g^{\prime}, r^{\prime}\right)\right) \subset \mathcal{W}:=\mathcal{W}(L, g, r) \subset T^{*} L
$$


Without loss of generality we assume in the following that we have perturbed $g$ so that there is a unique minimizing geodesic $\gamma$ in class $\beta$ and that theorem 3.2 applies to this class. Again $\beta^{\prime} \in H_{1}\left(L^{\prime} ; \mathbb{Z}\right), \beta:=\pi_{*} \circ \iota_{*} \beta^{\prime}$ and we aim at proving that $\mu_{L\left(L^{\prime}\right)}\left(\iota_{*} \beta^{\prime}\right)=0$. As before, we consider a sequence of almost complex structures $J_{n}$ on $\mathcal{W}$ that are cylindrical at infinity and that stretch the neck along the contact type hypersurface $\partial \mathcal{W}^{\prime} \subset T^{*} L$. By theorem 3.2 there exists for each $n$ a $J_{n}$-holomorphic map $u_{n}:(D \backslash\{0\}, \partial D) \longrightarrow(\mathcal{W}, L)$ which is asymptotic to the lift $\tilde{\gamma}$ of $\gamma$ at 0 . Letting $n$ go to infinity, we apply theorem 3.26 and get a limit holomorphic building. We focus henceforth on the unique component in the top floor $\mathcal{W} \backslash \mathcal{W}^{\prime}$ with a positive puncture. Since $L \subset \mathcal{W}^{\prime}$ and $\pi: \mathcal{W}^{\prime} \rightarrow L$ induces an isomorphism in homology, theorem 3.26 ensures that this component is a map $v: S^{2} \backslash Z \rightarrow \mathcal{W} \backslash \mathcal{W}^{\prime}$, where $Z \subset S^{2}$ is a finite set of punctures. Here $v$ is asymptotic at the unique positive puncture $z^{+} \in Z$ to the curve $\tilde{\gamma}$ and at the negative punctures $z_{i}^{-} \in Z$ to curves $\mathcal{I}\left(\tilde{\gamma}_{i}^{\prime}\right)$. The important point here is that $\tilde{\gamma}_{i}^{\prime}$ are lifts of geodesics of $L^{\prime}$ and that $\sum\left[\gamma_{i}^{\prime}\right]=\beta^{\prime}$ (this is seen as usual by considering $\pi \circ v$ as a cobordism between $\pi(\tilde{\gamma})=\gamma$ and $\left.\sum \pi \circ \iota\left(\gamma_{i}^{\prime}\right)\right)$. In particular, using lemma 3.25 the area of $v$ is given by $\mathcal{A}(v)=r \ell_{g}^{\min }(\beta)-\sum_{i} \lambda\left(\mathcal{I}\left(\tilde{\gamma}_{i}^{\prime}\right)\right)$. Moreover we have

$$
\begin{aligned}
\lambda\left(\mathcal{I}\left(\tilde{\gamma}_{i}^{\prime}\right)\right) & =\left[\lambda-\mathcal{I}_{*} \lambda^{\prime}\right]\left(\mathcal{I}\left(\tilde{\gamma}_{i}^{\prime}\right)\right)+\mathcal{I}_{*} \lambda^{\prime}\left(\mathcal{I}\left(\tilde{\gamma}_{i}^{\prime}\right)\right) \\
& =\left[\mathcal{I}^{*} \lambda-\lambda^{\prime}\right]\left(\tilde{\gamma}_{i}^{\prime}\right)+\lambda^{\prime}\left(\tilde{\gamma}_{i}^{\prime}\right) \\
& =\left[\mathcal{I}^{*} \lambda-\lambda^{\prime}\right]\left(\gamma_{i}^{\prime}\right)+r^{\prime} \ell_{g^{\prime}}\left(\gamma_{i}^{\prime}\right) \quad \text { (because } \mathcal{I}^{*} \lambda-\lambda^{\prime} \text { is closed) } \\
& =\iota^{*} \lambda\left(\left[\gamma_{i}^{\prime}\right]\right)+r^{\prime} \ell_{g^{\prime}}\left(\gamma_{i}^{\prime}\right) .
\end{aligned}
$$

Thus,

$$
\begin{aligned}
0<\mathcal{A}(v)=r \ell_{g}^{\min }(\beta)-\sum_{i} \lambda\left(\mathcal{I}\left(\tilde{\gamma}_{i}^{\prime}\right)\right) & =r \ell_{g}^{\min }(\beta)-\sum_{i}\left(\iota^{*} \lambda\left(\left[\gamma_{i}^{\prime}\right]\right)+r^{\prime} \ell_{g^{\prime}}\left(\gamma_{i}^{\prime}\right)\right) \\
& =r \ell_{g}^{\min }(\beta)-\iota^{*} \lambda\left(\beta^{\prime}\right)-\sum_{i} r^{\prime} \ell_{g^{\prime}}\left(\gamma_{i}^{\prime}\right) .
\end{aligned}
$$

This gives a bound on the total length of the multi-curve $\left\{\gamma_{i}^{\prime}\right\}$ that depends only on $\beta$. Since this multi-curve represents a given homology class, we see that, provided $g^{\prime}$ is chosen generically, it belongs to some finite set that depends only on $\beta$. Thus $v$ belongs to a set of maps whose asymptotics belong to a given finite set. Without loss of generality we assume that $\beta$ is a primitive class, so $v$ is somewhere injective. Moreover, we have total freedom in the choice of $J$ in some compact subset of $\mathcal{W} \backslash \overline{\mathcal{W}^{\prime}}$, which all possible $v$ must pass through. By standard transversality arguments we can therefore assume that the index of $v$ is nonnegative. This index is given by formula (22) in the appendix, corrected by the dimension $6 g-6+2 \# Z$ of the Teichmuller space of the domain of $v$ :

$$
\operatorname{ind}(v)=(n-3) \chi\left(S^{2} \backslash Z\right)+2 c_{1}^{\hat{\tau}}\left(v^{*} T\left(T^{*} L\right)\right)+\mu_{\mathrm{CZ}}^{\hat{\tau}}(\tilde{\gamma})-\sum_{i} \mu_{\mathrm{CZ}}^{\hat{\tau}}\left(\mathcal{I} \circ \tilde{\gamma}_{i}^{\prime}\right) .
$$

The precise definitions of these quantities are recalled in the appendix. The symbol $\hat{\tau}$ denotes an asymptotic symplectic trivialization of $v^{*} T\left(T^{*} L\right)$, i.e. choices of $2 n$ sections $\left(e_{1}, \ldots, e_{n}, f_{1}, \ldots, f_{n}\right)$ near the punctures $Z \subset S^{2}$ such that $\omega\left(e_{i}, e_{j}\right)=\omega\left(f_{i}, f_{j}\right)=0$ and $\omega\left(e_{i}, f_{j}\right)=\delta_{i j}$. The different quantities are Chern classes and Maslov indices of various objects computed with respect to this choice. In fact, the crucial data is the Lagrangian subbundle $\tau:=\left\langle e_{1}, \ldots, e_{n}\right\rangle$ of $\hat{\tau}$. In particular, the Conley-Zehnder indices depend only on 
$\tau$. Indeed, by [Gut12, Proposition 35], the difference of Conley-Zehnder indices computed in two different symplectic trivializations is given by twice the Maslov index of the loop of symplectic matrices that define the change of basis from one trivialization to the other. The latter can be expressed as the relative Maslov index of a reference Lagrangian distribution and its image under the change of basis along the loop. Assuming the two trivializations have the same reference Lagrangian distribution $\tau$, this Maslov index then vanishes (see for instance [MS95, Theorem 2.35]). In our setting, the vertical fiber distributions provide natural global Lagrangian distributions $\tau, \tau^{\prime}$ in $T\left(T^{*} L\right)$ and $T\left(T^{*} L^{\prime}\right)$. Since the domain of $v$ is a punctured sphere, $v^{*} \tau$ admits a Lagrangian trivialization $\left(e_{1}, \ldots, e_{n}\right)$, that can be completed to a symplectic trivialization $\hat{\tau}$ of $v^{*} T\left(T^{*} L\right)$ by adjunction of $\left(f_{1}=J e_{1}, \ldots, f_{n}=\right.$ $\left.J e_{n}\right)$, where $J$ is any compatible almost complex structure, e.g. $J=J_{g}$. Then $c_{1}^{\tau}(v)$ vanishes, while the computation in the proof of corollary A.7 shows that $\mu_{\mathrm{CZ}}^{\hat{\tau}}(\tilde{\gamma})=\mu_{\mathrm{CZ}}^{\tau}(\tilde{\gamma})=$ 0. By [Gut12, proposition 35],

$$
\mu_{\mathrm{CZ}}^{\tau}\left(\mathcal{I} \circ \tilde{\gamma}_{i}^{\prime}\right)=\mu_{\mathrm{CZ}}^{\mathcal{I}_{*} \tau^{\prime}}\left(\mathcal{I} \circ \tilde{\gamma}_{i}^{\prime}\right)+2 \mu_{\mathcal{I} \circ \tilde{\gamma}_{i}^{\prime}}\left(\mathcal{I}_{*} \tau^{\prime}, \tau\right)=\mu_{\mathrm{CZ}}^{\tau^{\prime}}\left(\tilde{\gamma}_{i}^{\prime}\right)+2 \mu_{\iota\left(L^{\prime}\right)}^{\tau}\left(\iota_{*}\left[\gamma_{i}^{\prime}\right]\right) .
$$

The equality of the first terms of the last equation is obvious because $\mathcal{I}$ is a symplectomorphism. For the second terms, notice that $\mathcal{I} \circ \tilde{\gamma}_{i}^{\prime}$ is homotopic to $\iota \circ \tilde{\gamma}_{i}^{\prime}$ in $\mathcal{W}^{\prime}$, where both $\mathcal{I}_{*} \tau^{\prime}$ and $\tau$ are well-defined Lagrangian distributions. So

$$
\mu_{\mathcal{I} \circ \tilde{\gamma}_{i}^{\prime}}\left(\mathcal{I}_{*} \tau^{\prime}, \tau\right)=\mu_{\iota \circ \gamma_{i}^{\prime}}\left(\mathcal{I}_{*} \tau^{\prime}, \tau\right)=\mu_{\iota \circ \gamma_{i}^{\prime}}\left(T \iota\left(L^{\prime}\right), \tau\right)=\mu_{\iota\left(L^{\prime}\right)}^{\tau}\left(\iota_{*}\left[\gamma_{i}^{\prime}\right]\right)
$$

The second equality holds by composition and the fact that $\mu_{\left\llcorner\circ \gamma_{i}^{\prime}\right.}\left(\mathcal{I}_{*} \tau^{\prime}, T \iota\left(L^{\prime}\right)\right)=\mu_{\gamma_{i}^{\prime}}\left(\tau^{\prime}, T L^{\prime}\right)=$ 0 . Getting back to the previous equation, and taking into account that $\mu_{\mathrm{CZ}}^{\tau^{\prime}}\left(\tilde{\gamma}_{i}^{\prime}\right)$ is nonnegative as the Morse index of a geodesic, we therefore get: (putting $\# Z=: k+2$ )

$$
\begin{aligned}
0 \leq \operatorname{ind}(v) & =(3-n) k-\sum_{i} \mu_{\mathrm{CZ}}^{\tau^{\prime}}\left(\tilde{\gamma}_{i}^{\prime}\right)-2 \sum_{i} \mu_{\iota\left(L^{\prime}\right)}^{\tau}\left(\iota_{*}\left[\gamma_{i}^{\prime}\right]\right) \\
& \leq(3-n) k-2 \mu_{\iota\left(L^{\prime}\right)}^{\tau}\left(\iota_{*} \beta^{\prime}\right) .
\end{aligned}
$$

When $n \geq 3$ we get $\mu_{\iota\left(L^{\prime}\right)}^{\tau}\left(\iota_{*} \beta^{\prime}\right) \leq 0$ and, replacing $\beta$ by $-\beta$ (which is still primitive) the vanishing of $\mu_{\iota\left(L^{\prime}\right)}^{\tau}\left(\iota_{*} \beta^{\prime}\right)$. When $n=1$, this vanishing is obvious.

We are therefore left with the case $n=2$, meaning that the manifolds $L$ and $L^{\prime}$ are surfaces. In this case, the fact that $L$ and $L^{\prime}$ have isomorphic integral homologies of degree 1 implies that they are diffeomorphic. Moreover, when $L$ is $S^{2}$ or $\mathbb{R P}^{2}$ there is again nothing to prove, so we assume that $L$ is either an orientable surface of genus at least 1 , or a connected sum of at least 2 projective planes. Then, there exists a metric met on $L$ and $L^{\prime}$ with non-positive curvature. Since

$$
L \subset \mathcal{I}\left(\mathcal{W}\left(L^{\prime}, g^{\prime}, \varepsilon^{\prime}\right)\right) \subset \mathcal{W}(L, g, r)
$$

one checks easily that after maybe extending $\mathcal{I}$, one may assume that

$$
L \subset \mathcal{I}\left(\mathcal{W}\left(L^{\prime}, \text { met }, \rho\right)\right) \subset \mathcal{W}(L, \text { met }, R),
$$

for appropriate $\rho$ and $R$. To see this, one checks that the 1 -form $\mathcal{I}_{*} \lambda^{\prime}$ extends to a Liouville 1 -form on $T^{*} L$, and uses this extension to embed $T^{*} L^{\prime}$ into $T^{*} L$. In other terms, we 
can assume that $L$ and $L^{\prime}$ are the same manifolds, equipped with the same metric $g=g^{\prime}$ with non-positive curvature. Remark now that the argument already used in theorem 3.21 and 3.26 shows that all but one negative puncture of $v$ are capped by some subbuilding (which are topological discs), so they are asymptotic to images by $\mathcal{I}$ of lifts of contractible geodesics of $L^{\prime}$. But since the metric $g^{\prime}$ has non-positive curvature, it has in particular no contractible geodesic, so $v$ has exactly one positive and one negative puncture. Our formula above therefore shows $\mu_{\iota\left(L^{\prime}\right)}^{\tau}\left(\iota_{*} \beta^{\prime}\right) \leq 0$. Again considering $-\beta$ instead of $\beta$, we obtain the vanishing of the Maslov index. This concludes our proof.

\subsection{Arnold's conjecture and the $\mathcal{C}^{0}$-rigidity of Lagrangian submanifolds}

Theorem 1 implies that the strongest form of $\mathcal{C}^{0}$-rigidity for Lagrangian submanifold follows from the Nearby Lagrangian conjecture, or even from the following weaker version:

Conjecture 4.1. Let $K \subset T^{*} L$ be an exact Lagrangian submanifold. If some Weinstein neighbourhood of $K$ contains the zero section, $K$ is Hamiltonian isotopic to $L$.

Proposition 4.2. Let $h: M \rightarrow M^{\prime}$ be a symplectic homeomorphism that takes a Lagrangian submanifold $L$ to a smooth submanifold $L^{\prime}$. Assume that conjecture 4.1 holds for $T^{*} L$. Then there exists a symplectic diffeomorphism $f: M \rightarrow M^{\prime}$ such that $f(L)=L^{\prime}$.

Proof: Let $h: M \rightarrow M^{\prime}$ be a symplectic homeomorphism that takes a Lagrangian submanifold $L$ to a Lagrangian submanifold $L^{\prime}$. We assume that conjecture 4.1 holds for $T^{*} L^{\prime}$ (considering $h^{-1}$ instead of $h$, this amounts to assuming that it holds for $T^{*} L$ ). Let $f_{n}: M \rightarrow M^{\prime}$ be a sequence of symplectic diffeomorphisms that approximate $h$ and put $L_{n}:=f_{n}(L)$. As explained in section 4.3, we have

$$
L^{\prime} \subset f_{n}(\mathcal{W}(L, g, \varepsilon)) \Subset \mathcal{W}\left(L^{\prime}, g^{\prime}, \varepsilon^{\prime}\right) \Subset \mathcal{W}\left(L^{\prime}, g^{\prime}, 1\right) \subset M^{\prime}
$$

for any $\varepsilon^{\prime} \ll 1, \varepsilon$ small enough compared with $\varepsilon^{\prime}$ and $n$ large enough. We need to find a symplectic diffeomorphism of $M^{\prime}$ that takes $L_{n}$ to $L^{\prime}$. We proceed as follows. Denote by $\lambda^{\prime}$ the natural Liouville form on $\mathcal{W}\left(L^{\prime}, g^{\prime}, 1\right) \subset M^{\prime}$ and $\pi: \mathcal{W}\left(L^{\prime}, g^{\prime}, 1\right) \rightarrow L^{\prime}$ the projection. For $n$ large enough it induces an isomorphism $\pi_{*}: H_{1}\left(L_{n}\right) \rightarrow H_{1}\left(L^{\prime}\right)$. The proof of theorem 3.a) shows that the cohomology class $a_{n}:=\left[\lambda_{\mid L_{n}}^{\prime}\right] \circ \pi_{*}^{-1} \in H^{1}\left(L^{\prime}\right)$ satisfies $\left\|a_{n}\right\| \leq C \varepsilon^{\prime}$. Moreover, the symplectic areas of the discs $D, h(D)$ and $f_{n}(D)$ with boundaries on $L, L^{\prime}$ and $L_{n}$, respectively, coincide by theorem 1.a), so if $\delta: H_{2}\left(M^{\prime}, L^{\prime}\right) \rightarrow H_{1}\left(L^{\prime}\right)$ is the connecting morphism, $a_{n \mid \operatorname{Im} \delta}$ vanishes. As a result, together with the exact sequence $H^{1}\left(M^{\prime}\right) \stackrel{r}{\longrightarrow}$ $H^{1}\left(L^{\prime}\right) \stackrel{\delta^{*}}{\longrightarrow} H^{2}\left(M^{\prime}, L^{\prime}\right)$, we see that $a_{n} \in \operatorname{Im}(r)$ and hence $a_{n}$ is the restriction to $H_{1}\left(L^{\prime}\right)$ of a cohomology class $A_{n} \in H^{1}\left(M^{\prime}\right)$. Let now $\theta_{n}$ be a closed 1-form on $M^{\prime}$ that represents $A_{n}$. Since $\mathcal{W}\left(L^{\prime}, g^{\prime}, 1\right)$ retracts to $L^{\prime}$, we can chose $\theta_{n}:=\pi^{*} \eta_{n}$ in $\mathcal{W}\left(L^{\prime}, g^{\prime}, 1\right)$ where $\eta_{n}$ is a closed 1-form on $L^{\prime}$ that represents the class $a_{n}$. Since $a_{n}$ is $\varepsilon^{\prime}$-small, we can choose $\eta_{n}$, and hence $\theta_{n}$ in $\mathcal{W}\left(L^{\prime}, g^{\prime}, 1\right)$ to be $\varepsilon^{\prime}$-small (in the uniform norm). Consider now the symplectic vector field on $M^{\prime}$ defined by

$$
\omega^{\prime}\left(X_{n}, \cdot\right)=-\theta_{n}
$$


and its time 1-map $\Phi_{n}$. Since $\theta_{n}$ is small on $\mathcal{W}\left(L^{\prime}, g^{\prime}, 1\right)$, we have $\Phi_{n}^{t}\left(\mathcal{W}\left(L^{\prime}, g^{\prime}, \varepsilon^{\prime}\right)\right) \subset$ $\mathcal{W}\left(L^{\prime}, g^{\prime}, 1\right)$ for all $0 \leq t \leq 1$, so $\Phi_{n \mid \mathcal{W}\left(L^{\prime}, g^{\prime}, \varepsilon^{\prime}\right)}$ coincides with the map $(q, p) \mapsto\left(q, p-\eta_{n}(q)\right)$. One thus verifies without difficulty that $\left[\lambda_{\mid \Phi_{n}\left(L_{n}\right)}^{\prime}\right]$ vanishes. Thus, $\Phi_{n}\left(L_{n}\right)$ is an exact Lagrangian submanifold in $\mathcal{W}\left(L^{\prime}, g^{\prime}, 1\right)$ when considered in $T^{*} L^{\prime}$. It is also easy to see that for $\varepsilon_{0}$ chosen small enough but fixed (so that $\left.\varepsilon^{\prime} \ll \varepsilon_{0}\right), \Phi_{n} \circ f_{n}\left(\mathcal{W}\left(L, g, \varepsilon_{0}\right)\right.$ ) is a Weinstein neighbourhood of $\Phi_{n}\left(L_{n}\right)$ that contains the zero section. If, as we assume in the statement of our proposition, conjecture 4.1 holds for $T^{*} L^{\prime}$, there exists a Hamiltonian diffeomorphism $\varphi$ in $T^{*} L^{\prime}$ which takes $\Phi_{n}\left(L_{n}\right)$ to $L^{\prime}$. A classical argument even shows that our Hamiltonian isotopy can also be modified so as to be supported in $\mathcal{W}\left(L^{\prime}, g^{\prime}, 1\right)$. As a result $\varphi$ can be seen as a Hamiltonian diffeomorphism of $M$ with support in $\mathcal{W}\left(L^{\prime}, g^{\prime}, 1\right)$, and the symplectomorphism $\varphi \circ \Phi_{n} \circ f_{n}: M \rightarrow M^{\prime}$ takes $L$ to $L^{\prime}$.

\section{$5 \quad$ Some embedding problems}

\subsection{A symplectic order on Riemannian metrics}

The set of Riemannian metrics $\mathcal{M e t}(M)$ on any manifold $M$ is endowed with a partial order defined by $g \leq g^{\prime}$ if and only if $g_{x}(v, v) \leq g_{x}^{\prime}(v, v)$ for all $x \in M$ and all $v \in T_{x} M$. This amounts to saying that for all $x \in M$, for all $p \in T_{x}^{*} M,\|p\|_{g^{\prime}} \leq\|p\|_{g}$, which in turn means that

$$
\mathcal{W}_{g} \subset \mathcal{W}_{g^{\prime}} \quad\left(\mathcal{W}_{g}:=\mathcal{W}(M, g, 1)=\left\{\|p\|_{g}<1\right\}\right) .
$$

In this perspective it is tempting to define a symplectic order on the space of Riemannian metrics in the following way.

Definition 5.1. We say that $g \prec_{\omega} g^{\prime}$ if there exists a symplectic embedding $\Phi: \mathcal{W}_{g} \hookrightarrow \mathcal{W}_{g^{\prime}}$ with $\Phi(M)=M$ and such that $\Phi_{\mid M}$ is homotopic to the identity, where $M$ is identified with the zero section of the cotangent bundle $T^{*} M$.

This relation is only a preorder because any diffeomorphism $f$ isotopic to the identity lifts to a symplectic diffeomorphism between $\mathcal{W}_{g}$ and $\mathcal{W}_{f_{*} g}$ that preserves the zero section, so $f_{*} g \prec_{\omega} g$ and $g \prec_{\omega} f_{*} g$ for all $g$. Thus $\prec_{\omega}$ induces another preorder $\prec_{\omega}$ on $\mathcal{M e t}(M) / \operatorname{Diff}^{0}(M)$. Knowing whether this new relation is now a partial order is related to subtle problems known as rigidity of metrics (see e.g. [CK94, BCG95] or [Cou14] for a contact analogue). The persistence of punctured holomorphic discs asymptotic to lifts of minimizing geodesics immediately implies a rigidity of this preorder. To state it recall that if $\beta \in H_{1}(M)$ we have defined

$$
\ell_{g}^{\min }(\beta)=\min \left\{\ell_{g}(\gamma) \mid \gamma \in \mathcal{C}^{1}\left(S^{1}, M\right),[\gamma]=\beta \in H_{1}(M)\right\}
$$

Theorem 5.2. Let $M$ be a closed manifold endowed with two Riemannian metrics $g$ and $g^{\prime}$ such that $g \prec_{\omega} g^{\prime}$. Then for all $\beta \in H_{1}(M)$ we have

$$
\ell_{g}^{\min }(\beta) \leq \ell_{g^{\prime}}^{\min }(\beta) \text {. }
$$


Proof: Let $g, g^{\prime}$ be two Riemannian metrics on $M$ with $g \prec_{\omega} g^{\prime}$. Let $\Phi: \mathcal{W}_{g} \hookrightarrow \mathcal{W}_{g^{\prime}}$ be a symplectic embedding such that $\Phi_{\mid M}$ is homotopic to the identity. For any $\varepsilon>0$ the map $\Phi$ embeds $\mathcal{W}_{g}$ compactly into $\mathcal{W}_{(1+\varepsilon) g^{\prime}}$. Let then $J_{n}$ be a sequence of cylindrical almost complex structures on $\mathcal{W}_{(1+\varepsilon) g^{\prime}}$ which stretch the neck of $\partial \Phi\left(\mathcal{W}_{g}\right)$. By theorem

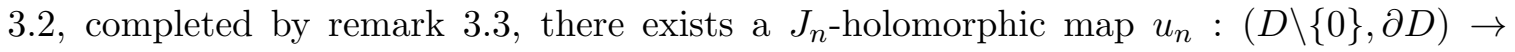
$\left(\mathcal{W}_{(1+\varepsilon) g^{\prime}}, M\right)$, asymptotic to a lift $\tilde{\gamma}^{\prime}$ of a geodesic $\gamma^{\prime}$ in the class $\beta$ that satisfies $\ell_{(1+\varepsilon) g^{\prime}}\left(\gamma^{\prime}\right) \leq$ $(1+2 \varepsilon) \ell_{g^{\prime}}^{\min }(\beta)$. A brief calculation shows that $\mathcal{A}\left(u_{n}\right)=\ell_{(1+\varepsilon) g^{\prime}}\left(\gamma^{\prime}\right)$. Since $\Phi_{\mid M}$ is homotopic to the identity, the projection $\pi: \partial \Phi\left(\mathcal{W}_{g}\right) \rightarrow M$ induces an isomorphism of homotopy groups, hence in homology, so we can apply theorem 3.26 exactly as in the second step of the proof of theorem 3.2 (§3.7) We get a limit holomorphic building $B$ that satisfies $\mathcal{A}(B)=\ell_{(1+\varepsilon) g^{\prime}}\left(\gamma^{\prime}\right)$. The holomorphic building contains a subbuilding $B^{\prime}$ which is a disc with boundary on $M$ and with one positive puncture asymptotic to a Reeb orbit $\tilde{\gamma}$ of $\partial \Phi\left(\mathcal{W}_{g}\right)$, whose projection to $M$ is a geodesic $\gamma$ that represents the class $\beta$. Since $\Phi(M)=$ $M$, we can apply lemma 3.25 in the exact setting and the symplectic area of the subbuilding $B^{\prime}$ is

$$
\mathcal{A}\left(B^{\prime}\right)=\ell_{g}(\gamma)
$$

We have $\mathcal{A}(B) \geq \mathcal{A}\left(B^{\prime}\right)$ and thus

$$
0 \leq \ell_{(1+\varepsilon) g^{\prime}}\left(\gamma^{\prime}\right)-\ell_{g}(\gamma) \leq(1+2 \varepsilon) \ell_{g^{\prime}}^{\min }(\beta)-\ell_{g}(\gamma)
$$

Thus, $\ell_{g}^{\min }(\beta) \leq \ell_{g}(\gamma) \leq(1+2 \varepsilon) \ell_{g^{\prime}}^{\min }(\beta)$. Since this estimate holds for all $\varepsilon$ we obtain the desired inequality.

It might be worth mentioning that theorem 5.2 can also be proved with symplectic homology, and might not be new at all (see [AS06, SW06] for the computation of the symplectic homology and spectral invariants of $\mathcal{W}(L, g, r)$ ). This paragraph is only meant for showing that the holomorphic punctured discs provided by theorem 3.2 allow to recover some of the quantitative invariants of $\mathcal{W}(L, g, r)$ obtained by spectral invariants associated to its symplectic homology.

\subsection{A Poisson bracket invariant}

In the previous section we saw that theorem 3.2 allowed us to study relative embeddings of Weinstein neighbourhoods of Lagrangian submanifolds one into another (relative means here that the Lagrangian submanifold has to be fixed by the symplectic embedding). It is also natural to consider symplectic embeddings of Weinstein neighbourhoods of the zero section into a general symplectic manifold and ask about the maximal symplectic size of such Weinstein neighbourhoods. In this general setting there are no punctured holomorphic discs of the ambient manifolds that can be exploited (although this may happen in some particular cases). The aim of this section is to define a monotone symplectic invariant of a Lagrangian embedding and to compute it explicitly for the zero section in certain Weinstein neighbourhoods in the cotangent bundle. This invariant is associated to a pair $(L, a)$ given by an embedded Lagrangian submanifold $L$ and a primitive integral cohomology 
class $a \in H^{1}(L ; \mathbb{Z})$. It is similar to the one defined in [EGM16] and is based on the Poisson bracket invariants of [BEP12]. By computing this invariant for a Lagrangian submanifold $L$ in a general symplectic manifold $M$ and using monotonicity, our computations here will provide information on the symplectic sizes of Weinstein neighborhoods of $L$ in $M$. Estimating the size of Weinstein neighbourhoods is a natural question and has been already considered in the literature, see for instance [Zeh13, CM18].

We start with the definition of our Poisson bracket invariant and then show how it relates to Lagrangian embeddings. Let $L \subset(M, \omega)$ be a connected closed Lagrangian submanifold, where $M$ is not necessarily compact. We associate to each non-zero primitive cohomology class $a \in H^{1}(L ; \mathbb{Z})$ a Poisson bracket invariant in the following way. By the de Rham isomorphism we can represent $a$ by a closed 1 -form $\theta$ on $L$. Now choose a base point $x_{0} \in L$. Define a function $\Theta: L \rightarrow \mathbb{R} / \mathbb{Z}$ as follows. For $x \in L$ set

$$
\Theta(x):=\int_{\gamma_{x}} \theta \bmod 1,
$$

where $\gamma_{x}$ is any smooth path in $L$ from $x_{0}$ to $x$. Since a different choice of path changes the integral by an integral value, this map is well defined. Consider the four sets given by

$$
X_{0}:=\Theta^{-1}\left(\left[0, \frac{1}{4}\right]\right), \quad Y_{0}:=\Theta^{-1}\left(\left[\frac{1}{4}, \frac{1}{2}\right]\right), \quad X_{1}:=\Theta^{-1}\left(\left[\frac{1}{2}, \frac{3}{4}\right]\right), \quad Y_{1}:=\Theta^{-1}\left(\left[\frac{3}{4}, 1\right]\right) .
$$

Then we have $X_{0} \cap X_{1}=Y_{0} \cap Y_{1}=\emptyset$ and $L=X_{0} \cup Y_{0} \cup X_{1} \cup Y_{1}$. Following the definition in [BEP12] we consider the set $\mathcal{F}\left(\theta, x_{0}\right)$ of all pairs $(H, K)$, where $H, K \in C_{c}^{\infty}(M)$ such that $\left.H\right|_{\mathrm{Op}\left(X_{0}\right)}=0,\left.H\right|_{\mathrm{Op}\left(X_{1}\right)}=1,\left.K\right|_{\mathrm{Op}\left(Y_{0}\right)}=0$ and $\left.K\right|_{\mathrm{Op}\left(Y_{1}\right)}=1$. We then set

$$
p b^{+}\left(L, \theta, x_{0}\right):=\inf _{\mathcal{F}\left(\theta, x_{0}\right)} \max _{M}\{H, K\} \in[0, \infty) .
$$

We now define (cf. [EGM16])

$$
b p(L, a):=\frac{1}{\inf \left\{p b^{+}\left(L, \theta, x_{0}\right) \mid[\theta]=a, x_{0} \in L\right\}} \in(0, \infty] .
$$

We also write $b p(L, a, M)$ when we want to emphasize the ambient symplectic manifold. When $a$ runs through the set of primitive classes of $H^{1}(L ; \mathbb{Z})$ the numbers $b p(L, a)$ provide a set of quantitative invariants for Lagrangian submanifolds. More precisely, if $\varphi:(M, \omega) \rightarrow$ $\left(M^{\prime}, \omega^{\prime}\right)$ is a symplectomorphism that maps a Lagrangian submanifold $L$ to $L^{\prime}:=\varphi(L)$, then $b p\left(L, \varphi^{*} a^{\prime}, M\right)=b p\left(L^{\prime}, a^{\prime}, M^{\prime}\right)$. These invariants are obviously monotone: if two symplectic manifolds $M$ and $M^{\prime}$ contain Lagrangian submanifolds $L$ and $L^{\prime}$ and there exists a relative symplectic embedding $f:(M, L) \hookrightarrow\left(M^{\prime}, L^{\prime}\right)$, then

$$
b p\left(L,\left(f_{\mid L}\right)^{*} a^{\prime}, M\right) \leq b p\left(L^{\prime}, a^{\prime}, M^{\prime}\right)
$$

for all primitive $a^{\prime} \in H^{1}\left(L^{\prime} ; \mathbb{Z}\right)$. A version of these invariants was introduced and computed in several examples in [EGM16]. There a list of properties of $b p$ is given and the definition is extended to include non-primitive $a$, which is also possible in our case. In the rest of this section we compute these invariants for the zero section in Weinstein neighborhoods in cotangent bundles. Before we state our result we recall the stable norm of a cohomology class together with an estimate of this number using closed curves. 
Definition 5.3. For a Riemannian manifold $(L, g)$ we endow its cohomology group $H^{1}(L ; \mathbb{R})$ with the stable norm

$$
\|a\|_{\text {st }}:=\inf _{[\theta]=a} \max _{q \in L}\|\theta(q)\|_{g} .
$$

Then ([Gro99, Proposition 4.35]),

$$
\|a\|_{\mathrm{st}}=\sup \left\{\frac{a([\gamma])}{\ell_{g}(\gamma)} \mid \gamma \in \mathcal{C}^{1}\left(S^{1}, L\right), a([\gamma])>0\right\} .
$$

The main result of this section is theorem 4 , that we recall now:

Theorem 4. Let $(L, g)$ be a closed Riemannian manifold and $\mathcal{W}(L, g, r):=\left\{\|p\|_{g}<r\right\} \subset$ $T^{*} L$. Let also $a \in H^{1}(L ; \mathbb{Z})$ be a primitive class. Then we have

$$
b p(L, a, \mathcal{W}(L, g, r))=\frac{r}{\|a\|_{\text {st }}}=r \inf \left\{\frac{\ell_{g}(\gamma)}{a([\gamma])} \mid \gamma \in \mathcal{C}^{1}\left(S^{1}, L\right), a([\gamma])>0\right\} .
$$

By monotonicity these numerical invariants provide bounds for the symplectic size of Weinstein neighborhoods of Lagrangian embeddings. To put it in a general framework, consider for a Lagrangian submanifold $L \subset(M, \omega)$ with a given Riemannian metric $g$ on $L$ the quantity

$$
c_{(M, L)}(L, g):=\sup \{r>0 \mid(\mathcal{W}(L, g, r), L) \stackrel{\omega}{\hookrightarrow}(M, L)\} .
$$

This is a relative version of the embedding capacity $c^{(M, \omega)}(L, g)$ defined and studied in [CM18, p.9] (the embedding of $L$ for $c^{(M, \omega)}(L, g)$ is not fixed). We have the inequality $c_{(M, L)}(L, g) \leq c^{(M, \omega)}(L, g)^{-1}$.

Corollary 5.4. Let $L \subset M$ be a Lagrangian submanifold and $g$ a metric on $L$. If for $r>0$ there is a relative symplectic embedding $(\mathcal{W}(L, g, r), L) \hookrightarrow(M, L)$, then for all primitive classes $a \in H^{1}(L ; \mathbb{Z}) \backslash\{0\}$ we have

$$
r \leq b p(L, a, M) \cdot\|a\|_{\mathrm{st}} .
$$

In other terms,

$$
c_{(M, L)}(L, g) \leq \inf _{a \in H^{1}(L) \backslash\{0\}} b p(L, a, M) \cdot\|a\|_{\mathrm{st}} .
$$

This corollary relies on the lower bound for $b p(L, a, \mathcal{W}(L, g, r))$ which, as will be clear from the proof, only requires soft techniques. The hard part in using this corollary therefore really lies in obtaining an upper bound for $b p(L, a, M)$.

As an illustration of equation (16), let us prove the following:

Corollary 5.5. Let $\left(\mathbb{C P}^{n}, \omega\right)$ be normalized such that the class $\left[\mathbb{C P}^{1}\right]$ has area 1 and let $L_{\text {Cliff denote the Clifford torus. Then }}$

$$
c_{\left(\mathbb{C P}^{n}, L_{\mathrm{Cliff}}\right)}\left(\mathbb{T}^{n}, g\right)=\frac{1}{\sqrt{n}(n+1)}
$$


Proof: We endow $\mathbb{T}^{n}=\mathbb{R}^{n} / \mathbb{Z}^{n}$ with the flat metric $g$ and consider the parametrization of $L_{\text {Cliff }}$ by $\mathbb{T}^{n}$ given by $\left(t_{1}, \ldots, t_{n}\right) \mapsto\left[e^{2 i \pi t_{1}}: \cdots: e^{2 i \pi t_{n}}: 1\right]$. The standard Hamiltonian $\mathbb{T}^{n}$-action on $\mathbb{C P}^{n}$ gives us a moment map $\Phi: \mathbb{C P}^{n} \rightarrow\left(\mathbb{R}^{n}\right)^{*}$ whose image is the simplex

$$
\Delta:=\left\{\left(x_{1}, \ldots, x_{n}\right) \in\left(\mathbb{R}^{n}\right)^{*} \mid x_{1}, \ldots, x_{n} \geq 0,0 \leq x_{1}+\ldots+x_{n} \leq 1\right\},
$$

and $L_{\text {Cliff }}=\Phi^{-1}(\mathbf{x})$ for $\mathbf{x}:=\left(\frac{1}{n+1}, \ldots, \frac{1}{n+1}\right)$. Note that we have a canonical isomorphism $\left(\mathbb{Z}^{n}\right)^{*} \simeq H^{1}\left(\mathbb{T}^{n}\right)$ that we identify with $H^{1}\left(L_{\text {Cliff }}\right)$ via our specific embedding. Then, for the class $a:=(1, \ldots, 1) \in H^{1}\left(\mathbb{T}^{n}\right)$ one can show that $b p\left(L_{\text {Cliff }}, a, \mathbb{C P}^{n}\right) \leq 1 /(n(n+1))$ by applying an adapted version of theorem 1.4 together with the computation in theorem 2.16 of [EGM16]. We also easily see that for the flat metric we have $\|a\|_{\text {st }}=\sqrt{n}$, so

$$
c_{\left(\mathbb{C P}^{n}, L_{\mathrm{Cliff}}\right)}\left(\mathbb{T}^{n}, g\right) \leq \frac{1}{\sqrt{n}(n+1)} .
$$

Now note that $\Phi^{-1}$ (int $\Delta$ ) is symplectomorphic to $\mathbb{T}^{n} \times \operatorname{int} \Delta \subset T^{*} \mathbb{T}^{n}$, and that there is an explicit symplectic embedding of $\mathcal{W}\left(\mathbb{T}^{n}, g, r_{\max }\right)$ into $\mathbb{T}^{n} \times \operatorname{int} \Delta$ relative to $\mathbb{T}^{n} \times\{\mathbf{x}\}$, with $r_{\max }:=\frac{1}{\sqrt{n}(n+1)}=d_{\text {eucl }}(\mathbf{x}, \partial \Delta)$. This provides a relative symplectic embedding of $\left(\mathcal{W}\left(\mathbb{T}^{n}, g, r_{\max }\right), 0_{\mathbb{T}^{n}}\right)$ into $\left(\mathbb{C P}^{n}, L\right)$, thus we obtain the lower bound $c_{\left(\mathbb{C P}^{n}, L\right)}\left(\mathbb{T}^{n}, g\right) \geq$ $\frac{1}{\sqrt{n}(n+1))}$.

Proof of theorem 4: As is common with Poisson bracket invariants (cf. [BEP12, EGM16]), we obtain an upper bound from the persistence of holomorphic punctured discs under a deformation of the almost complex structure (theorem 3.2). For the lower bound we provide an explicit construction.

Step I: upper bound. Consider first a curve $\gamma$ in a class $\beta \in H_{1}(L)$ that satisfies $a(\beta)>0$. We can freely perturb $g$ in the $\mathcal{C}^{0}$-topology for this proof, since the assumption on $g$ implies a symplectic embedding $\left(\mathcal{W}\left(L, g^{\prime}, r-\varepsilon\right), L\right) \hookrightarrow(M, L)$ for any metric $g^{\prime} \varepsilon$-close to $g$. So we can assume that $g$ has a unique minimal geodesic $\gamma(\beta)$ in the class $\beta$ and a unique $J_{g}$-holomorphic map $u_{\gamma, g}$, as defined on page 12 , which is Fredholm regular. Fix a closed 1-form $\theta$ that represents the class $a$ and a base point $x_{0} \in L$. This choice fixes the sets $X_{0}, Y_{0}, X_{1}, Y_{1}$ as above. Let $(H, K) \in \mathcal{F}\left(\theta, x_{0}\right)$ be a pair of smooth functions supported in $\mathcal{W}\left(L, g, r^{\prime}\right)$ for some $r^{\prime}<r$. Since $H, K$ are constant in neighborhoods of $X_{i}$ and $Y_{i}$ we have $d H \wedge d K \equiv 0$ on $L$ and hence $H d K$ is a closed 1-form on $L$. Following the calculation in [EGM16, Theorem 3.4] we see that $\left[\left.H d K\right|_{L}\right]=a \in H^{1}(L)$ and the exact form

$$
\omega_{s}:=\omega+s d H \wedge d K
$$

is symplectic for all $s \in I:=\left[0,1 / \max _{M}\{H, K\}\right)$. Note that $\omega_{s}$ coincides with the symplectic form $\omega=d \lambda$ near the boundary. Thus there exists a generic smooth family of almost complex structures $\left\{J_{s}\right\}_{s \in I}$ in $\mathcal{W}(L, g, r)$ compatible with $\omega_{s}, g$-cylindrical at infinity and starting at $J_{0}=\Phi^{*} J_{g}$ (where $\Phi: \mathcal{W}(L, g, r) \rightarrow T^{*} L$ is the map defined in section 3.6.3). Since there is a unique $J_{0}$-holomorphic punctured disc asymptotic to $\tilde{\gamma}(\beta)$ and it is regular, 
there is a family $\left\{u_{s}\right\}$ of $J_{s}$-holomorphic punctured discs asymptotic to $\tilde{\gamma}(\beta)$ for small $s \geq 0$. The proof of theorem 3.2 (section 3.7) shows that this family persists for all $s \in I$. Indeed, the main ingredient is the compactness theorem 3.21 , which only relies on the fact that $\tilde{\gamma}(\beta)$ has least action among the lifts of the curves in class $\beta$ and this still holds for $\omega_{s}$. Thus, for all $s \in I$ there exists a $J_{s}$-holomorphic map $u_{s}:(D \backslash\{0\}, \partial D) \rightarrow(\mathcal{W}(L, g, r), L)$ which is asymptotic to $\tilde{\gamma}(\beta)$. Then using Stokes' Theorem we have

$$
0<\int u_{s}^{*} \omega_{s}=\int u_{s}^{*} d \lambda+s \int u_{s}^{*} d H \wedge d K=r \ell_{g}(\gamma(\beta))-s a(\beta)
$$

Thus we have $s<\frac{r \ell_{g}(\gamma(\beta))}{a(\beta)}=\frac{r \ell_{g}^{\min }(\beta)}{a(\beta)}$. This holds for all $s \in I$, hence

$$
\frac{1}{\max _{M}\{H, K\}} \leq \frac{r \ell_{g}^{\min }(\beta)}{a(\beta)}
$$

Varying $(H, K) \in \mathcal{F}\left(\theta, x_{0}\right)$ as well as $\theta$ in the class of $a$ and the choice of base point $x_{0} \in L$, we see that

$$
b p(L, a, \mathcal{W}(L, g, r)) \leq r \frac{\ell_{g}^{\min }(\beta)}{a(\beta)} \leq r \frac{\ell_{g}(\gamma)}{a([\gamma])} .
$$

Step II: lower bound. It is enough to find good functions. We first prove the weaker inequality

$$
b p(L, a, \mathcal{W}(L, g, r)) \geq \frac{r}{2\|a\|_{\mathrm{st}}},
$$

because the proof is more visual. We then get rid of the constant $\frac{1}{2}$. Figure 4 represents the different functions that appear in the proof.
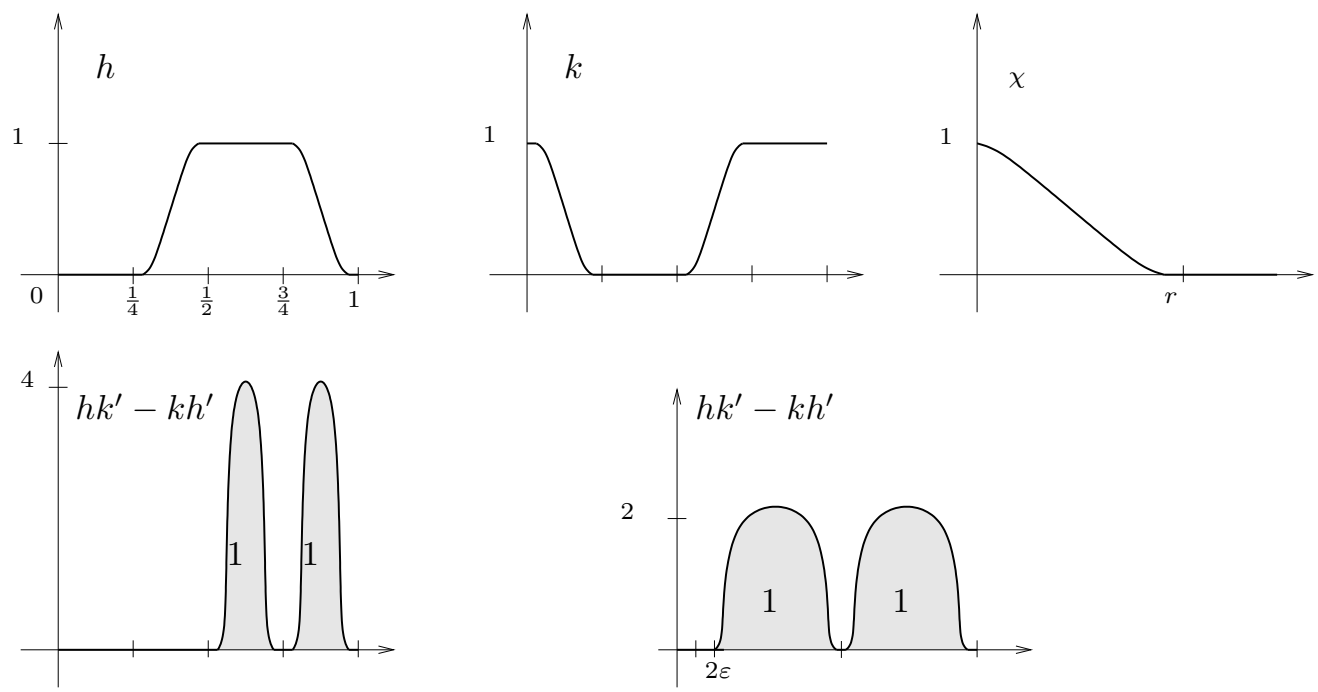

Figure 4: The almost optimal functions for the $b p$-invariant.

Let $\theta$ be a closed 1-form that represents the class $a$ with $\|\theta\|_{g}<\|a\|_{\text {st }}+\varepsilon$ and $\Theta$ its $\mathbb{R} / \mathbb{Z}$-valued primitive as defined above. Let $h, k: \mathbb{R} / \mathbb{Z} \rightarrow[0,1]$ be smooth functions such 
that $h$ equals 0 on $\left[0, \frac{1}{4}\right]$ and 1 on $\left[\frac{1}{2}, \frac{3}{4}\right]$ and $k$ equals 0 on $\left[\frac{1}{4}, \frac{1}{2}\right]$ and 1 on $\left[\frac{3}{4}, 1\right]$. Let also $\chi:[0, r) \rightarrow[0,1]$ be a smooth, compactly supported function that equals 1 near 0 . We define the compactly supported functions $H, K: \mathcal{W}(L, g, r) \rightarrow[0,1]$ as follows. For canonical coordinates $(q, p) \in T^{*} L$ we set

$$
\begin{aligned}
& H(q, p):=\chi\left(\|p\|_{g}\right) h \circ \Theta(q) \\
& K(q, p):=\chi\left(\|p\|_{g}\right) k \circ \Theta(q) .
\end{aligned}
$$

Then

$$
\{H, K\}(q, p)=\chi\left(\|p\|_{g}\right) h \circ \Theta(q)\left\{\chi\left(\|p\|_{g}\right), k \circ \Theta(q)\right\}+\chi\left(\|p\|_{g}\right) k \circ \Theta(q)\left\{h \circ \Theta(q), \chi\left(\|p\|_{g}\right)\right\}
$$

Now for any function $f(q)$ we have

$$
\left\{f(q), \chi\left(\|p\|_{g}\right)\right\}=\sum_{i=1}^{n} \frac{\partial f(q)}{\partial q_{i}} \frac{\partial \chi\left(\|p\|_{g}\right)}{\partial p_{i}}=\chi^{\prime}\left(\|p\|_{g}\right) \sum_{i=1}^{n} \frac{\partial f(q)}{\partial q_{i}} \frac{\partial\|p\|_{g}}{\partial p_{i}} .
$$

For a point $q_{0} \in L$ we choose coordinates in a neighborhood such that the metric $g$ satisfies $g_{i j}\left(q_{0}\right)=\delta_{i j}$. Then at $q_{0}$ have $\frac{\partial\|p\|_{g}}{\partial p_{i}}=\frac{p_{i}}{\|p\|_{g}}$. Applying this to our case we get at $q_{0}$

$$
\{H, K\}\left(q_{0}, p\right)=\chi\left(\|p\|_{g}\right) \chi^{\prime}\left(\|p\|_{g}\right)\left(h^{\prime} k-h k^{\prime}\right) \circ \Theta\left(q_{0}\right) \sum_{i=1}^{n} \frac{\partial \Theta\left(q_{0}\right)}{\partial q_{i}} \frac{p_{i}}{\|p\|_{g}}
$$

Note that $\frac{\partial \Theta(q)}{\partial q_{i}}=\theta_{q}\left(\partial_{q_{i}}\right)$ and $\sum_{i} \frac{p_{i}}{\|p\|_{g}} \partial_{q_{i}}:=v$ has norm $\|v\|_{g}=1$ for $p \neq 0$. By our choice of $\theta$ we have $\left|\theta_{q_{0}}(v)\right|<\|a\|_{\text {st }}+\varepsilon$. We therefore get

$$
\{H, K\}\left(q_{0}, p\right) \leq\left|\left(\frac{\chi^{2}}{2}\right)^{\prime}\right| \cdot\left|\left(h^{\prime} k-h k^{\prime}\right) \circ \Theta\left(q_{0}\right)\right| \cdot\left(\|a\|_{\mathrm{st}}+\varepsilon\right) .
$$

Now notice that because of the properties of $h, k$ (see also figure 4 ),

$$
h^{\prime} k-h k^{\prime}= \begin{cases}h^{\prime} & \text { on }\left[\frac{3}{4}, 1\right] \\ -k^{\prime} & \text { on }\left[\frac{1}{2}, \frac{3}{4}\right] \\ 0 & \text { else. }\end{cases}
$$

Taking into account that the only constraint on $h^{\prime}, k^{\prime}$ is that their integral equals \pm 1 on $\left[\frac{3}{4}, 1\right]$ and $\left[\frac{1}{2}, \frac{3}{4}\right]$, respectively, we see that for a good choice of $h, k$ we have $\left\|h^{\prime} k-h k^{\prime}\right\|_{\mathcal{C}^{0}} \leq 4+\varepsilon$. Also, since the only constraint on $\chi$ is that $\chi$ varies from 1 to 0 within the interval $[0, r)$, we see that for a good choice of $\chi$ (for which $\chi^{2}$ is almost linear) we can ensure that $\left\|\left(\chi^{2} / 2\right)^{\prime}\right\|_{\mathcal{C}^{0}} \leq \frac{1}{2 r}+\varepsilon$. Altogether we get

$$
\{H, K\}\left(q_{0}, p\right) \leq\left(\frac{1}{2 r}+\varepsilon\right)(4+\varepsilon)\left(\|a\|_{\mathrm{st}}+\varepsilon\right) \leq \frac{2\|a\|_{\mathrm{st}}}{r}+C \varepsilon
$$

for $\varepsilon \ll 1$ and a constant $C$. The choice of $q_{0} \in L$ was arbitrary and $\varepsilon>0$ can be chosen arbitrarily small. Thus,

$$
b p(L, a, \mathcal{W}(L, g, r)) \geq \frac{r}{2\|a\|_{\mathrm{st}}} .
$$


Finally, in order to get rid of the constant $\frac{1}{2}$ we use an equivalent definition of $b p(L, a, M)$. Namely for the function $\Theta$ one can choose the four sets

$$
X_{0}^{\prime}:=\Theta^{-1}([0, \varepsilon]), \quad Y_{0}^{\prime}:=\Theta^{-1}([\varepsilon, 2 \varepsilon]), \quad X_{1}^{\prime}:=\Theta^{-1}\left(\left[2 \varepsilon, \frac{1}{2}\right]\right), \quad Y_{1}^{\prime}:=\Theta^{-1}\left(\left[\frac{1}{2}, 1\right]\right),
$$

and choose the functions $H, K \in C_{c}^{\infty}(M)$ to satisfy $H_{\mid \mathrm{Op}\left(X_{0}^{\prime}\right)}=0, H_{\mid \mathrm{Op}\left(X_{1}^{\prime}\right)}=1, K_{\mid \mathrm{Op}\left(Y_{0}^{\prime}\right)}=$ 0 and $K_{\mid \mathrm{Op}\left(Y_{1}^{\prime}\right)}=1$ in the definition of $b p(L, a, M)$. Indeed, if $\rho: \mathbb{R} / \mathbb{Z} \rightarrow \mathbb{R} / \mathbb{Z}$ is a diffeomorphism that equals the identity near 0 and sends the intervals $[0, \varepsilon],[\varepsilon, 2 \varepsilon],\left[2 \varepsilon, \frac{1}{2}\right]$ and $\left[\frac{1}{2}, 1\right]$ to $\left[0, \frac{1}{4}\right],\left[\frac{1}{4}, \frac{1}{2}\right],\left[\frac{1}{2}, \frac{3}{4}\right]$ and $\left[\frac{3}{4}, 1\right]$, then as in [EGM16, Section 3.2] one can show that $\rho \circ \Theta=: \Theta^{\prime}=\int \theta^{\prime}$ for a closed 1-from $\theta^{\prime}$ representing the class $a$. Thus pairs of functions in $\mathcal{F}\left(\theta^{\prime}, x_{0}\right)$ are equivalent to pairs $(H, K)$ with the corresponding values on $X_{i}^{\prime}$ and $Y_{j}^{\prime}$. Since for $b p(L, a, M)$ the infimum is taken over all $[\theta]=a$, we see that this definition is equivalent to the original one.

For this extremalization problem the result is slightly better, since now $h^{\prime} k-h k^{\prime}$ is supported on $\left[2 \varepsilon, \frac{1}{2}\right] \cup\left[\frac{1}{2}, 1\right]$ and we can choose the slopes of $h$ and $k$ to be close to $\pm \frac{1}{2}$ on

these intervals. For a suitable choice of $h$ and $k$ we therefore have $\left\|h^{\prime} k-h k^{\prime}\right\|_{\mathcal{C}^{0}} \leq 2+\varepsilon$, and the inequality (17) becomes

$$
\{H, K\} \leq\left(\frac{1}{2 r}+\varepsilon\right)(2+\varepsilon)\left(\|a\|_{\mathrm{st}}+\varepsilon\right) \leq \frac{\|a\|_{\mathrm{st}}}{r}+C \varepsilon
$$

Since $\varepsilon>0$ can be chosen arbitrarily small, we indeed conclude that $b p(L, a, \mathcal{W}(L, g, r)) \geq$ $r\|a\|_{\mathrm{st}}^{-1}$.

\section{A Transversality for punctured holomorphic disks}

In this section we prove generic transversality for moduli spaces of punctured pseudoholomorphic curves with boundary in symplectic cobordisms. This result is essentially folklore and the statement follows mostly from standard results in the literature where parts have already been done (see [Dra04, MS12, Wen10, HWZ99]). Nevertheless, as far as we are aware, transversality has not been explicitly written for punctured surfaces with totally real boundary conditions in symplectic cobordisms of arbitrary dimension, so we provide a proof for the sake of completeness. This is mostly a matter of compiling the different sources cited above. For the case without boundary we refer the reader to the excellent exposition by Wendl [Wen16].

We fix notation. Let $(X, \omega)$ be a symplectic cobordism. Thus $(X, \omega)$ is a symplectic manifold containing a compact domain $K \subset X$ with contact boundary such that $(X \backslash \operatorname{int}(K), \omega)$ is symplectomorphic to the union $\left([0, \infty) \times M_{+}, d\left(e^{r} \alpha_{+}\right)\right) \sqcup\left((-\infty, 0] \times M_{-}, d\left(e^{r} \alpha_{-}\right)\right)$for suitable contact manifolds $\left(M_{+}, \alpha_{+}\right)$and $\left(M_{-}, \alpha_{-}\right)$. Here $r$ denotes the $\mathbb{R}$-coordinate. The contact manifolds $M_{ \pm}$may contain several components and one, but not both, may be empty. Let $L \subset(X, \omega)$ be a compact Lagrangian submanifold contained in $\operatorname{int}(K)$. We fix a Riemannian metric $g$ that is translation-invariant outside a compact set containing $K$ and 
such that $L$ is totally geodesic. Let $\gamma$ be a non-degenerate Reeb orbit of a contact manifold $(M, \alpha)$ with period $T>0$. Define $Z_{+}:=[0, \infty) \times S^{1}$ and $Z_{-}:=(-\infty, 0] \times S^{1}$ with the coordinates $(s, t)$ and conformal structure $j \partial_{s}=\partial_{t}$. We say that a map $v=(a, u): Z_{+} \rightarrow \mathbb{R} \times M$ is positively asymptotic to $\gamma$, if $\lim _{s \rightarrow \infty} a(s, t)=\infty$ and $\lim _{s \rightarrow \infty} u(s, t)=\gamma(T t)$. We say a map $v^{\prime}=\left(a^{\prime}, u^{\prime}\right): Z_{-} \rightarrow \mathbb{R} \times M$ is negatively asymptotic to $\gamma$, if $\lim _{s \rightarrow-\infty} a^{\prime}(s, t)=-\infty$ and $\lim _{s \rightarrow-\infty} u^{\prime}(s, t)=\gamma(T t)$. Here we assume uniform convergence of the limits. For a fixed Riemann surface $(\Sigma, j)$ (possibly with boundary) let $\Gamma=\left\{z_{1}, \ldots, z_{l+m}\right\} \subset \operatorname{int}(\Sigma)$ be a set of punctures. Given a family of non-degenerate Reeb orbits $\mathcal{O}_{\Gamma}=\left\{\gamma_{1}^{+}, \ldots, \gamma_{l}^{+}, \gamma_{1}^{-}, \ldots, \gamma_{m}^{-}\right\}$ in $M_{ \pm}$, we say that a map $\varphi: \Sigma \backslash \Gamma \rightarrow X$ is asymptotic to the family $\mathcal{O}_{\Gamma}$, if for all $i$ there exists a disk neighborhood $\mathcal{U}_{i}$ of $z_{i}$ in $\Sigma$, a biholomorphism $\psi_{i}: Z_{ \pm} \rightarrow \mathcal{U}_{i} \backslash\left\{z_{i}\right\}$ such that $\varphi \circ \psi_{i}: Z_{ \pm} \rightarrow X$ is asymptotic to $\gamma_{i}^{ \pm}$.

Let us fix an almost complex structure $J_{\mathrm{Cyl}}$ on $X$ that is compatible with $\alpha_{ \pm}$outside a compact neighbourhood of $K$. Denote by $\mathcal{J}_{\mathrm{Cyl}}^{\infty}\left(J_{\text {Cyl }}\right)$ the space of smooth almost complex structures on $X$ that are compatible with $\omega$ and coincide with $J_{\mathrm{Cyl}}$ outside this fixed compact neighbourhood of $K$. We endow this space with the $C^{\infty}$-topology. Then $\mathcal{J}_{\mathrm{Cyl}}^{\infty}\left(J_{\mathrm{Cyl}}\right)$ is a contractible space. In order to lighten notation we omit the $J_{\text {Cyl }}$ and only write $\mathcal{J}_{\text {Cyl }}^{\infty}$. In this appendix, this notation will always refer to $\mathcal{J}_{\text {Cyl }}^{\infty}\left(J_{\text {Cyl }}\right)$. For a chosen $J \in \mathcal{J}_{\text {Cyl }}^{\infty}$ and family $\mathcal{O}_{\Gamma}$ we define the moduli space of punctured Riemann surfaces with boundary on $L$,

$$
\mathcal{M}\left(\mathcal{O}_{\Gamma}, J\right):=\left\{\begin{array}{l|c}
u:(\Sigma \backslash \Gamma, \partial \Sigma ; j) \longrightarrow(X, L ; J) & d u+J \circ d u \circ j=0, u(\partial \Sigma) \subset L, \\
u \text { is asymptotic to } \mathcal{O}_{\Gamma}
\end{array}\right\} .
$$

We also consider the subset $\mathcal{M}^{*}\left(\mathcal{O}_{\Gamma}, J\right)$ consisting of curves in $\mathcal{M}\left(\mathcal{O}_{\Gamma}, J\right)$ that are somewhere injective in $\operatorname{int}(K)$, i.e. elements $u$ for which there exists a $z \in \operatorname{int}(\Sigma \backslash \Gamma)$ such that $d u(z) \neq 0$, $u(z) \in \operatorname{int}(K)$ and $u^{-1}(u(z))=\{z\}$. The main result of this section is as follows.

Theorem A.1. There exists a subset $\mathcal{J}^{\text {reg }} \subset \mathcal{J}_{\text {Cyl }}^{\infty}$ such that the following holds:

1. If $J \in \mathcal{J}^{\text {reg }}$, then $\mathcal{M}^{*}\left(\mathcal{O}_{\Gamma}, J\right)$ is a smooth manifold of dimension

$$
\begin{aligned}
\operatorname{dim}\left(\mathcal{M}^{*}\left(\mathcal{O}_{\Gamma}, J\right)\right)= & n \chi(\dot{\Sigma})+2 c_{1}^{\tau}\left(u^{*} T X\right)+\mu^{\tau}\left(u^{*} T X, u^{*} T L\right) \\
& +\sum_{i=1}^{l} \mu_{\mathrm{CZ}}^{\tau}\left(\gamma_{i}^{+}\right)-\sum_{j=1}^{m} \mu_{\mathrm{CZ}}^{\tau}\left(\gamma_{j}^{-}\right)+\# \Gamma,
\end{aligned}
$$

locally around the element $u \in \mathcal{M}^{*}\left(\mathcal{O}_{\Gamma}, J\right)$. Here $\tau$ denotes a trivialization of $u^{*} T X$ on the cylindrical ends and boundary.

2. The subset $\mathcal{J}^{\text {reg }}$ is of second category in $\mathcal{J}_{\mathrm{Cyl}}^{\infty}$.

We refer the reader to the end of section A.1 for the definitions of the topological invariants $c_{1}^{\tau}, \mu^{\tau}$ and $\mu_{\mathrm{CZ}}^{\tau}$. We call the elements $J \in \mathcal{J}^{\text {reg }}$ regular. For our applications we need to discuss the dependence of $\mathcal{M}^{*}\left(\mathcal{O}_{\Gamma}, J\right)$ under variations of $J \in \mathcal{J}^{\text {reg }}$. For a smooth path $\left\{J_{t}\right\}_{t \in[0,1]} \subset \mathcal{J}_{\mathrm{Cyl}}^{\infty}$ define

$$
\mathcal{W}^{*}\left(\mathcal{O}_{\Gamma},\left\{J_{t}\right\}\right)=\left\{(t, u) \mid t \in[0,1], u \in \mathcal{M}^{*}\left(\mathcal{O}_{\Gamma}, J_{t}\right)\right\} .
$$

For two regular $J_{0}, J_{1} \in \mathcal{J}^{\text {reg }}$ we denote by $\mathcal{J}\left(J_{0}, J_{1}\right)$ the space of all smooth paths in $\mathcal{J}_{\text {Cyl }}^{\infty}$ connecting $J_{0}$ to $J_{1}$. 
Theorem A.2. There exists a subset $\mathcal{J}^{\mathrm{reg}}\left(J_{0}, J_{1}\right) \subset \mathcal{J}\left(J_{0}, J_{1}\right)$ such that the following holds:

1. If $\left\{J_{t}\right\}_{t \in[0,1]} \in \mathcal{J}^{\mathrm{reg}}\left(J_{0}, J_{1}\right)$, then $\mathcal{W}^{*}\left(\mathcal{O}_{\Gamma},\left\{J_{t}\right\}\right)$ is a smooth oriented manifold with boundary

$$
\partial \mathcal{W}^{*}\left(\mathcal{O}_{\Gamma},\left\{J_{t}\right\}\right)=\mathcal{M}^{*}\left(\mathcal{O}_{\Gamma}, J_{0}\right) \cup \mathcal{M}^{*}\left(\mathcal{O}_{\Gamma}, J_{1}\right) .
$$

2. The set $\mathcal{J}^{\mathrm{reg}}\left(J_{0}, J_{1}\right)$ is of second category in $\mathcal{J}\left(J_{0}, J_{1}\right)$.

The proof of theorem A.1 follows the standard line of arguments in the literature, see e.g. [MS12]. We first recall various notions from the general theory of Cauchy-Riemann type operators on punctured Riemann surfaces. In the functional analytical setup we explain the Banach manifolds and bundles involved in our setting. We then introduce the universal moduli space as a Banach submanifold of the aforementioned Banach manifolds and use this construction to prove theorem A.1. Adaptations of these arguments then provide a proof of theorem A.2.

\section{A.1 CR type operators on Hermitian bundles over punctured surfaces}

Let $(\Sigma, j)$ be a compact Riemann surface of genus $g$ with $m \geq 0$ boundary components. Choose a non-empty finite set $\Gamma \subset \operatorname{int}(\Sigma)$ of positive and negative interior punctures, we write $\Gamma=\Gamma^{+} \cup \Gamma^{-}$. The punctured surface is then denoted $\dot{\Sigma}=\Sigma \backslash \Gamma$. Now for every puncture $z \in \Gamma^{ \pm}$we choose a closed neighborhood $\mathcal{U}_{z} \subset \Sigma$ of $z$ together with a biholomorphic map $\varphi_{z}:\left(\dot{\mathcal{U}}_{z}, j\right) \rightarrow\left(Z_{ \pm}, i\right)$, where $\dot{\mathcal{U}}_{z}:=\mathcal{U}_{z} \backslash\{z\}$ is the punctured neighborhood and $Z_{+}=$ $[0, \infty) \times S^{1}, Z_{-}=(-\infty, 0] \times S^{1}$ are complex cylinders. Note that the assumption that $\varphi_{z}$ is a biholomorphism implies that $\varphi_{z}(w) \rightarrow \pm \infty$ when $w \rightarrow z$. The union of punctured neighborhoods $\dot{\mathcal{U}}_{z}$ will be called the cylindrical ends of $\dot{\Sigma}$.

Let $(E, \omega, J) \rightarrow(\dot{\Sigma}, j)$ be a smooth Hermitian vector bundle of rank $n$ over the punctured surface. By Hermitian structure we mean that $(E, \omega)$ is a symplectic vector bundle and $J$ is an $\omega$-compatible almost complex structure. The inner product is then given by

$$
\langle\cdot, \cdot\rangle_{E}:=\omega(\cdot, J \cdot)+i \omega(\cdot, \cdot) .
$$

We call a trivialization $\Phi$ of $E$ near $z \in \Gamma^{ \pm}$admissible, if $\Phi:\left.E\right|_{\dot{\mathcal{U}}_{z}} \rightarrow Z_{ \pm} \times \mathbb{C}^{n}$ is a unitary bundle isomorphism which projects to the biholomorphism $\varphi_{z}$. Here $\mathbb{C}^{n}$ is identified with the standard Hermitian vector space. Note that every Hermitian vector bundle $E$ over $\dot{\Sigma}$ has admissible trivializations over the cylindrical ends. Furthermore, there exist admissible trivializations that extend over $\dot{\Sigma}$, since $\Gamma \neq \emptyset$ and thus $\dot{\Sigma}$ has the homotopy type of a 1-dimensional cellular complex. For a choice of admissible trivialization $\Phi$ near all $z \in \Gamma$ we fix a volume form $d v o l$ on $\dot{\Sigma}$ such that $d v o l$ is equal to $d s \wedge d t$ in the cylindrical coordinates induced by $\Phi$.

Let $\left(E^{\prime}, \omega^{\prime}, J^{\prime}\right)$ and $(E, \omega, J)$ be two Hermitian vector bundles over $\dot{\Sigma}$. We denote by $\operatorname{Hom}^{1,0}\left(E^{\prime}, E\right)$ and $\operatorname{Hom}^{0,1}\left(E^{\prime}, E\right)$ the corresponding complex vector bundles consisting of complex linear and antilinear bundle maps $E^{\prime} \rightarrow E$. Sometimes we include the complex structures $J, J^{\prime}$ in the notation to provide more clarity. In the following we will often 
consider the bundle $F:=\operatorname{Hom}^{0,1}(T \dot{\Sigma}, E)$ (and sections thereof). In particular this bundle inherits a Hermitian structure $(F, \bar{\omega}, \bar{J})$ by setting

$$
\bar{J} \eta=J \circ \eta, \quad \bar{\omega}_{p}\left(\eta, \eta^{\prime}\right)=\frac{\omega_{p}\left(\eta(v), \eta^{\prime}(v)\right)}{d v o l(v, j v)},
$$

for $p \in \dot{\Sigma}, \eta, \eta^{\prime} \in F$ and any non-zero choice of $v \in T_{p} \dot{\Sigma}$. Furthermore, any admissible trivialization $\Phi$ of $(E, \omega, J)$ induces an admissible trivialization of $(F, \bar{\omega}, \bar{J})$ over $\bigcup_{z \in \Gamma} \dot{\mathcal{U}}_{z}$ via

$$
\left.F\right|_{\dot{\mathcal{U}}_{z}} \rightarrow Z_{ \pm} \times \mathbb{C}^{n}, \quad \eta \longmapsto \Phi\left(\eta\left(\partial_{s}\right)\right)
$$

where $\partial_{s}$ is the vector field on $\left.T \dot{\Sigma}\right|_{\dot{\mathcal{U}}_{z}}$ arising from $\varphi_{z}$. On $E$ and $F$ we define the $L^{2}$-inner product to be

$$
\begin{aligned}
\langle\eta, \xi\rangle_{L^{2}(E)} & :=\int_{\dot{\Sigma}} \omega(\eta, J \xi) d v o l \quad \text { for } \eta, \xi \in C_{0}^{\infty}(E), \\
\langle\nu, \rho\rangle_{L^{2}(F)} & :=\int_{\dot{\Sigma}} \bar{\omega}(\nu, \bar{J} \rho) d v o l \quad \text { for } \nu, \rho \in C_{0}^{\infty}(F) .
\end{aligned}
$$

We recall the theory of Cauchy-Riemann type operators on Hermitian bundles over punctured surfaces. We begin with the notion of an asymptotic operator.

Let $\left(S^{1} \times \mathbb{R}^{2 n}, \omega_{0}, J_{0}\right) \rightarrow S^{1}$ be the standard Hermitian vector bundle of complex rank $n$. An asymptotic operator $\mathbf{A}$ on $S^{1} \times \mathbb{R}^{2 n}$ is any real linear differential operator

$$
\mathbf{A}: C^{\infty}\left(S^{1}, \mathbb{R}^{2 n}\right) \longrightarrow C^{\infty}\left(S^{1}, \mathbb{R}^{2 n}\right), \quad \eta \longmapsto-J_{0} \partial_{t} \eta-S(t) \eta,
$$

where $S: S^{1} \rightarrow \operatorname{End}\left(\mathbb{R}^{2 n}\right)$ is any smooth loop of symmetric matrices. Equivalently, an asymptotic operator $\mathbf{A}$ is any operator of the form $-J_{0} \nabla_{t}$, where $\nabla$ is a symplectic connection on $\left(S^{1} \times \mathbb{R}^{2 n}, \omega_{0}\right)$. For the real $L^{2}$-bundle metric given by

$$
\left\langle\eta, \eta^{\prime}\right\rangle_{L^{2}}:=\int_{S^{1}} \omega_{0}\left(\eta(t), J_{0} \eta^{\prime}(t)\right) d t
$$

the operator $\mathbf{A}$ is symmetric. If we consider $\mathbf{A}$ as a bounded linear operator $H^{1}\left(S^{1}, \mathbb{R}^{2 n}\right) \rightarrow$ $L^{2}\left(S^{1}, \mathbb{R}^{2 n}\right)$, then $\mathbf{A}$ is Fredholm with index equal to 0 (see e.g. [Wen16]) . We say that A is non-degenerate, if its spectrum $\sigma(\mathbf{A})$ does not contain 0 . In this case the operator $\mathbf{A}: H^{1}\left(S^{1}, \mathbb{R}^{2 n}\right) \rightarrow L^{2}\left(S^{1}, \mathbb{R}^{2 n}\right)$ induces an isomorphism.

We recall the standard $\bar{\partial}$ operator for smooth functions on $(\dot{\Sigma}, j)$,

$$
\bar{\partial}: C^{\infty}(\dot{\Sigma}, \mathbb{C}) \longrightarrow \Omega^{0,1}(\dot{\Sigma}, \mathbb{C}), \quad f \longmapsto d f+i \circ d f \circ j
$$

Definition A.3. Let $(E, \omega, J)$ be a smooth Hermitian vector bundle over $(\dot{\Sigma}, j)$. A real linear first order differential operator $\mathbf{D}: \Gamma(E) \rightarrow \Gamma\left(\operatorname{Hom}^{0,1}(T \dot{\Sigma}, E)\right)$ is called of CauchyRiemann type, if it satisfies

$$
\mathbf{D}(f \eta)=\bar{\partial}(f) \eta+f \mathbf{D} \eta
$$


for every $\eta \in \Gamma(E)$ and $f \in C^{\infty}(\dot{\Sigma}, \mathbb{C})$. For a puncture $z \in \Gamma$ we say that $\mathbf{D}$ is asymptotic to an asymptotic operator $\mathbf{A}_{z}$ at $z$, if in an admissible trivialization near $z$ the operator $\mathbf{D}$ takes the form

$$
\mathbf{D} \eta(s, t) \frac{\partial}{\partial s}=\partial_{s} \eta(s, t)+J_{0} \partial_{t} \eta(s, t)+S(s, t) \eta(s, t),
$$

where $S(s, t)$ is a smooth family in $\operatorname{End}_{\mathbb{R}}\left(\mathbb{C}^{n}\right)$ such that $S(s, t)$ converges uniformly as $s \rightarrow$ $\pm \infty$ to a smooth loop of symmetric matrices $S_{z}(t)$, where $-J_{0} \partial_{t}-S_{z}(t)$ is the expression of $\mathbf{A}_{z}$ in the trivialization.

Let $k \geq 1$ and $p>2$. For a smooth Hermitian vector bundle $(E, \omega, J) \rightarrow(\dot{\Sigma}, j)$ with fixed admissible trivialization at the cylindrical ends we consider the topological vector space $W_{\mathrm{loc}}^{k, p}(E)$ of $W_{\text {loc }}^{k, p}$-sections of $E$. We define the Banach space

$$
W^{k, p}(E):=\left\{\eta \in W_{\mathrm{loc}}^{k, p}(E) \mid \eta_{z} \in W^{k, p}\left(\operatorname{int}\left(Z_{ \pm}\right), \mathbb{C}^{n}\right) \forall z \in \Gamma^{ \pm}\right\},
$$

where $\eta_{z}$ is $\left.\eta\right|_{\dot{\mathcal{U}}_{z}}$ in coordinates induced by the admissible trivialization and the area form $d v o l$ is used to define the $W^{k, p}$-norm of $\eta_{z}$. By choosing a compact set $C$ that contains $\dot{\Sigma} \backslash \bigcup \dot{\mathcal{U}}_{z}$ we obtain a Banach space norm by summing the corresponding $W^{k, p}$-norms over $C$ and the cylindrical ends. Norms that arise in this way are equivalent. Now for a smooth totally real subbundle $\left.\Lambda \subset E\right|_{\partial \Sigma}$ we consider the Banach subspace

$$
W_{\Lambda}^{k, p}(E):=\left\{\eta \in W^{k, p}(E) \mid \eta(\partial \Sigma) \subset \Lambda\right\}
$$

We recall the following statement on Cauchy-Riemann type operators, see [Sch95, Wen10].

Theorem A.4. Let $\mathbf{A}_{z}$ be asymptotic operators for each $z \in \Gamma$ and let

$$
\mathbf{D}: W_{\Lambda}^{k, p}(E) \longrightarrow W^{k-1, p}\left(\operatorname{Hom}^{0,1}(T \dot{\Sigma}, E)\right)
$$

be a Cauchy-Riemann type operator asymptotic to $\mathbf{A}_{z}$ for each $z$. Then $\mathbf{D}$ is a Fredholm operator if all $\mathbf{A}_{z}$ are non-degenerate. Moreover, $\operatorname{ind}(\mathbf{D})$ and $\operatorname{ker}(\mathbf{D})$ are independent of $k$ and $p$.

In order to compute the index of the Fredholm operator $\mathbf{D}$ we briefly recall certain topological invariants.

Let $(E, J) \rightarrow(\Sigma, j)$ be a complex vector bundle over a compact Riemann surface $\Sigma$ with boundary $\partial \Sigma$. Let $\tau:\left.E\right|_{\partial \Sigma} \rightarrow \partial \Sigma \times \mathbb{C}^{n}$ be a trivialization over the boundary. We define the relative first Chern class with respect to the trivialization $\tau$ as follows. If $(E, J)$ is a line bundle, then $c_{1}^{\tau}(E)$ counts the zeros (with signs) of a generic smooth section that is non-zero and constant over $\partial \Sigma$ with respect to $\tau$. For two complex vector bundles $\left(E_{1}, J_{1}\right)$ and $\left(E_{2}, J_{2}\right)$ with trivializations $\tau_{1}$ and $\tau_{2}$ over $\partial \Sigma$ we set $c_{1}^{\tau_{1} \oplus \tau_{2}}\left(E_{1} \oplus E_{2}\right)=c_{1}^{\tau_{1}}\left(E_{1}\right)+$ $c_{1}^{\tau_{2}}\left(E_{2}\right)$. Since every complex vector bundle over a Riemann surface splits into a sum of line bundles this uniquely determines $c_{1}^{\tau}(E)$ for all complex vector bundles. The definition then extends to complex vector bundles over punctured Riemann surfaces where $\tau$ equals a chosen admissible trivialization on the cylindrical ends. For a totally real subbundle $\left.\Lambda \subset E\right|_{\partial \Sigma}$ the trivialization $\tau$ also defines a Maslov index $\mu^{\tau}(E, \Lambda) \in \mathbb{Z}$, see [MS12]. 
Now let $\mathbf{A}_{z}$ be an asymptotic operator for $z \in \Gamma$. In the admissible trivialization we have $\mathbf{A}_{z}=-J_{0} \partial_{t}-S(t)$. Let $\Psi(t) \in \operatorname{Sp}(n)$ be a smooth loop of symplectic matrices satisfying the differential equation $\dot{\Psi}(t)=J_{0} S(t) \Psi(t)$. If $\mathbf{A}_{z}$ is non-degenerate, then $\Psi(1)$ does not have 1 as an eigenvalue and we can consider its Conley-Zehnder index $\mu_{\mathrm{CZ}}^{\tau}(\Psi(t))$, see [Sal99]. We then set the Conley-Zehnder index of $\mathbf{A}_{z}$ to be $\mu_{\mathrm{CZ}}^{\tau}\left(\mathbf{A}_{z}\right):=\mu_{\mathrm{CZ}}^{\tau}(\Psi(t))$.

We recall the following statement from [Wen10].

Theorem A.5. Let $\mathbf{A}_{z}$ be non-degenerate asymptotic operators for each $z \in \Gamma$ and let

$$
\mathbf{D}: W_{\Lambda}^{k, p}(E) \longrightarrow W^{k-1, p}\left(\operatorname{Hom}^{0,1}(T \dot{\Sigma}, E)\right)
$$

be a Cauchy-Riemann type operator asymptotic to $\mathbf{A}_{z}$ for each $z$. Then the Fredholm index of $\mathbf{D}$ is

$$
\operatorname{ind}(\mathbf{D})=n \chi(\dot{\Sigma})+2 c_{1}^{\tau}(E)+\mu^{\tau}(E, \Lambda)+\sum_{z \in \Gamma^{+}} \mu_{\mathrm{CZ}}^{\tau}\left(\mathbf{A}_{z}\right)-\sum_{z \in \Gamma^{-}} \mu_{\mathrm{CZ}}^{\tau}\left(\mathbf{A}_{z}\right),
$$

where $n=\operatorname{rank}_{\mathbb{C}}(E)$.

Note that the dependence of equation (20) on the choice of trivialization $\tau$ cancels out. In subsequent sections we will consider Banach spaces of sections of $E$ with exponential weights at the punctures. Let $(E, \omega, J) \rightarrow(\dot{\Sigma}, j)$ be a Hermitian vector bundle over a punctured Riemann surface equipped with an admissible trivialization. For $k \in \mathbb{N}, p>2$ and $\delta \in \mathbb{R}$ we define the Banach space

$$
W_{\Lambda}^{k, p, \delta}(E) \subset W_{\mathrm{loc}}^{k, p}(E)
$$

to be the space of sections $\eta \in W_{\text {loc }}^{k, p}(E)$ whose restriction to the neighborhood of any puncture $z \in \Gamma^{ \pm}, \eta_{z}: Z_{ \pm} \rightarrow \mathbb{C}^{n}$, satisfies

$$
\left\|e^{ \pm \delta s} \eta_{z}\right\|_{W^{k, p}\left(Z_{ \pm}\right)}<\infty
$$

and $\eta(\partial \Sigma) \subset \Lambda$. Thus for $\delta=0$ we recover the Banach space $W_{\Lambda}^{k, p}(E)$ from before and for $\delta>0$ the sections in $W_{\Lambda}^{k, p, \delta}(E)$ are guaranteed to have exponential decay at infinity. Note that for $k=0$, the dual space $\left(L^{p, \delta}(E)\right)^{*}$ is isomorphic to $L^{q,-\delta}(E)$ for $1 / p+1 / q=1$ via the $L^{2}$-inner product.

\section{A.2 Functional analytic setup}

We describe the Banach spaces used in the proof of Theorems A.1 and A.2. Let $k \in \mathbb{N}$, $p>2$ and $\delta>0$ and let $\mathcal{O}_{\Gamma}=\left\{\gamma_{z}\right\}_{z \in \Gamma}$ be a collection of Reeb orbits of $M_{ \pm}$, one for every puncture $z \in \Gamma=\Gamma^{+} \cup \Gamma^{-}$. We define the space

$$
\mathcal{B}^{k, p, \delta}:=\mathcal{B}^{k, p, \delta}\left(\dot{\Sigma}, \partial \Sigma ; X, L ; \mathcal{O}_{\Gamma}\right)
$$

to consist of maps $u: \dot{\Sigma} \rightarrow X$ of class $W_{\text {loc }}^{k, p}$ which satisfy $u(\partial \Sigma) \subset L$ and have asymptotically cylindrical behavior approaching $\gamma_{z}$ at the puncture $z \in \Gamma^{ \pm}$. Basically, one can view $u$ as a 
decreasing perturbation of $y(s, t):=\left(T s, \gamma_{z}(T t)\right)$ up to a shift in the cylindrical ends. To be precise, this means that in cylindrical coordinates $(s, t) \in Z_{ \pm}$near $z$, there exist constants $s_{0}$ and $t_{0}$ such that for sufficiently large $|s|$ we have

$$
u\left(s+s_{0}, t+t_{0}\right)=\exp _{y(s, t)} h(s, t),
$$

where $h \in W^{k, p, \delta}\left(y^{*} T H_{ \pm}\right)$and the exponential map is defined with respect to any $\mathbb{R}$ invariant metric on $H_{ \pm}:=\mathbb{R} \times M_{ \pm}$. The condition $k p>2$ implies via the Sobolev embedding theorem that $\mathcal{B}^{k, p, \delta} \hookrightarrow C^{0}(\dot{\Sigma}, X)$. Even though $\dot{\Sigma}$ is non-compact, we can still give the space $\mathcal{B}^{k, p, \delta}$ the structure of a smooth, separable and metrizable Banach manifold by generalizing the results of [Elí67].

$$
T_{u} \mathcal{B}^{k, p, \delta}=W_{\Lambda}^{k, p, \delta}\left(u^{*} T X\right) \oplus V,
$$

where the summands are defined as follows. $\Lambda$ is the Lagrangian subbundle

$$
\Lambda:=\left(\left.u\right|_{\partial \Sigma}\right)^{*} T L \longrightarrow \partial \Sigma,
$$

so that sections $v \in W_{\Lambda}^{k, p, \delta}\left(u^{*} T W\right)$ are required to decay exponentially near the puncture and satisfy $v(\partial \Sigma) \subset \Lambda$. $V$ is a $2(\# \Gamma)$-dimensional real vector space with basis given by noncanonical choices of two sections $\dot{\Sigma} \rightarrow u^{*} T W$ supported in $\mathcal{U}_{z}$ for every puncture $z \in \Gamma$ and asymptotic to the vector fields $\partial_{r}, R_{\alpha_{ \pm}}$of $T\left(\mathbb{R} \times M_{ \pm}\right)$. In particular, $V$ contains vector fields that are asymptotically parallel to orbit cylinders $y(s, t)=\left(T s, \gamma_{z}(T t)\right)$ in the cylindrical ends.

Let $J \in \mathcal{J}_{\text {cyl }}^{\infty}$. Note that by [HWZ96, Theorem 1.5], for a finite family of non-degenerate orbits $\mathcal{O}_{\Gamma}$ there exists an open set $I \subset \mathbb{R}$ containing 0 , such that for all $\delta \in I$ maps satisfying $d u+J \circ d u \circ i=0$ and the asymptotic condition at $\mathcal{O}_{\Gamma}$ automatically satisfy the exponential decay estimate near each puncture. Hence for $\delta$ sufficiently small the space $\mathcal{B}^{k, p, \delta}$ contains all pseudoholomorphic maps asymptotic to a chosen finite family of Reeb orbits.

For $l \geq k$ consider now the space $\mathcal{J}^{l}$ of almost complex structures of class $C^{l}$ on $X$, compatible with the symplectic structure and cylindrical at infinity. Since $\mathcal{J}^{l}$ consists of almost complex structures that are translation invariant outside of a compact set, we can give $\mathcal{J}^{l}$ the structure of a smooth separable Banach manifold by using the translation invariant metric. For $J \in \mathcal{J}^{l}$ the tangent space $T_{J} \mathcal{J}^{l}$ consists of compactly supported $C^{l}$-sections $Y$ of the smooth bundle $\operatorname{End}(T X, J, \omega) \rightarrow X$ that satisfy

$$
Y J+J Y=0, \quad \omega(Y v, w)+\omega(v, Y w)=0 .
$$

The first equation is derived from the condition $J^{2}=-\mathrm{Id}$ and the second equation comes from the compatibility of $J$ with $\omega$. The space of such sections is a Banach space and provides a local chart containing $J$ via the mapping $Y \mapsto J \exp (-J Y)$.

We now consider the bundle $\mathcal{E}^{k-1, p, \delta} \rightarrow \mathcal{B}^{k, p, \delta} \times \mathcal{J}^{l}$, whose fiber over $(u, J)$ is given by

$$
\mathcal{E}_{(u, J)}^{k-1, p, \delta}:=W^{k-1, p, \delta}\left(\operatorname{Hom}^{0,1}\left((T \dot{\Sigma}, j),\left(u^{*} T X, J\right)\right)\right),
$$

the space of complex-antilinear bundle maps. One can show that $\mathcal{E}^{k-1, p, \delta}$ has the structure of a $C^{l-k}$ Banach space bundle, see the proof of Proposition 3.2.1 in [MS12]. The map 
$\mathcal{F}: \mathcal{B}^{k, p, \delta} \times \mathcal{J}^{l} \rightarrow \mathcal{E}^{k-1, p, \delta}$ given by

$$
\mathcal{F}(u, J):=d u+J \circ d u \circ j
$$

then defines a $C^{l-k}$-section of the bundle, because $\mathcal{F}(u, J) \in \mathcal{E}^{k-1, p, \delta}$ satisfies the exponential weighting condition when $J$ is translation invariant on the ends. The zeros of this section is given by the union of the moduli spaces $\mathcal{M}\left(\mathcal{O}_{\Gamma}, J\right)$ for $J \in \mathcal{J}^{l}$. For $(u, J)$ such that $\mathcal{F}(u, J)=0$ the vertical differential $D \mathcal{F}(u, J): T_{u} \mathcal{B}^{k, p, \delta} \times T_{J} \mathcal{J}^{l} \rightarrow \mathcal{E}_{(u, J)}^{k-1, p, \delta}$ of the section $\mathcal{F}$ is given by [MS12, Section 3.2]:

$$
D \mathcal{F}(u, J)(\xi, Y):=\nabla \xi+J \circ \nabla \xi \circ j+\left(\nabla_{\xi} J\right) \circ d u \circ j+Y(u) \circ d u \circ j,
$$

where $\nabla$ is any symmetric connection on $X$. For fixed $J \in \mathcal{J}^{l}$ we also consider the restriction of $\mathcal{F}$ to $\mathcal{B}^{k, p, \delta}$ and the associated vertical differential $\mathbf{D}_{u}: T_{u} \mathcal{B}^{k, p, \delta} \rightarrow \mathcal{E}_{(u, J)}^{k-1, p, \delta}$ at the zero section,

$$
\mathbf{D}_{u}(\xi)=\nabla \xi+J \circ \nabla \xi \circ j+\left(\nabla_{\xi} J\right) \circ d u \circ j .
$$

The smooth manifold structure of $\mathcal{M}^{*}\left(\mathcal{O}_{\Gamma}, J\right)$ depends on the properties of the map $\mathbf{D}_{u}$ as a Fredholm operator. We continue with the analysis of this operator. Let $\tau$ be an admissible trivialization of $u^{*} T X$ on the cylindrical ends and boundary of $\dot{\Sigma}$.

Proposition A.6. For $\delta>0$ sufficiently small the operator $\mathbf{D}_{u}: T_{u} \mathcal{B}^{k, p, \delta} \rightarrow \mathcal{E}_{(u, J)}^{k-1, p, \delta}$ is Fredholm and has index

$$
\operatorname{ind}\left(\mathbf{D}_{u}\right)=n \chi(\dot{\Sigma})+2 c_{1}^{\tau}\left(u^{*} T X\right)+\mu^{\tau}\left(u^{*} T X, \Lambda\right)+\sum_{i=1}^{m} \mu_{\mathrm{CZ}}^{\tau}\left(\gamma_{i}^{+}\right)-\sum_{j=1}^{n} \mu_{\mathrm{CZ}}^{\tau}\left(\gamma_{j}^{-}\right)+\# \Gamma \text {. }
$$

Proof: Since $T_{u} \mathcal{B}^{k, p, \delta}=W_{\Lambda}^{k, p, \delta}\left(u^{*} T X\right) \oplus V$ and $V$ is finite dimensional, it suffices to prove that $\mathbf{D}_{u}$ restricted to $W_{\Lambda}^{k, p, \delta}\left(u^{*} T X\right)$ is Fredholm. By abuse of notation let $\mathbf{D}_{u}$ be the restricted operator. We proceed by showing that $\mathbf{D}_{u}$ is conjugate to a Cauchy-Riemann type operator with non-degenerate asymptotic operators at the punctures.

Let $z \in \Gamma^{ \pm}$be a puncture and suppose $\mathbf{D}_{u}(\cdot) \frac{\partial}{\partial s}$ takes the form $\bar{\partial}+S(s, t)$ in the coordinates of the admissible trivialization on the cylindrical end of $z$, where $S(s, t)$ converges uniformly as $s \rightarrow \pm \infty$ to a smooth loop of symmetric matrices $S_{z}(t)$. A computation reveals (see the proof of Theorem 3.6 of [Dra04]) that $S_{z}(t)=0_{2 \times 2} \oplus S_{\gamma_{z}}(t)$, where we use the splitting in the trivialization arising from $T\left(\mathbb{R} \times M_{ \pm}\right) \simeq \beta \oplus \xi_{ \pm}$. Here $\beta$ is the complex line bundle generated by $\partial_{r}$ and the Reeb vector field $R_{\alpha_{ \pm}}$and $\xi_{ \pm}$is the pullback of the contact distribution on $M_{ \pm}$. The matrix $S_{\gamma_{z}}(t)$ is derived from the linearization of the Reeb flow at $\gamma_{z}$ and is symmetric and non-singular since $\gamma_{z}$ is non-degenerate. Now since $S_{z}(t)$ is zero on the complex line bundle $\beta$, this implies that the associated asymptotic operator $\mathbf{A}_{z}:=-J_{0} \partial_{t}-S_{z}(t)$ is degenerate. In particular the operator (for $\delta=0$ )

$$
\mathbf{D}_{u}: W_{\Lambda}^{k, p}\left(u^{*} T X\right) \longrightarrow W^{k-1, p}\left(\operatorname{Hom}^{0,1}\left(T \dot{\Sigma}, u^{*} T X\right)\right)
$$

is not Fredholm. This issue is resolved by considering spaces with exponential weights. 
Let $(E, \omega, J)$ be a Hermitian vector bundle over $\dot{\Sigma}$. For $\delta \in \mathbb{R}$ pick a smooth function $f: \dot{\Sigma} \rightarrow \mathbb{R}$ such that $f( \pm s, t)=\mp \delta s$ on the cylindrical ends. Then we obtain Banach space isomorphisms

$$
\Phi_{\delta}: W^{k, p}(E) \rightarrow W^{k, p, \delta}(E), \quad \eta \mapsto e^{f} \eta, \quad \Psi_{\delta}: W^{k-1, p, \delta}(E) \rightarrow W^{k-1, p}(E), \quad \theta \mapsto e^{f} \theta .
$$

Returning to our setting we consider the bounded linear map

$$
\mathbf{D}_{u}^{\prime}:=\Psi_{\delta}^{-1} \mathbf{D}_{u} \Phi_{\delta}: W_{\Lambda}^{k, p}\left(u^{*} T X\right) \rightarrow W^{k-1, p}\left(\operatorname{Hom}^{0,1}\left(T \dot{\Sigma}, u^{*} T X\right)\right) .
$$

We see that $\mathbf{D}_{u}^{\prime}$ is a linear Cauchy-Riemann type operator on $\dot{\Sigma}$. Moreover a short calculation reveals that at a puncture $z \in \Gamma^{ \pm}$the operator $\mathbf{D}_{u}^{\prime}$ takes the form

$$
\mathbf{D}_{u}^{\prime} \eta=\bar{\partial} \eta+\left(S(s, t) \mp \delta \operatorname{Id}_{2 n \times 2 n}\right) \eta
$$

in the trivialization and is therefore asymptotic to the operator

$$
\mathbf{A}_{z}^{\prime}:=-J_{0} \partial_{t}-S_{z}(t) \pm \delta \operatorname{Id}_{2 n \times 2 n}=\mathbf{A}_{z} \pm \delta \operatorname{Id}_{2 n \times 2 n}
$$

The spectrum of $\mathbf{A}_{z}$ is discrete. Thus for $\delta>0$ chosen small enough, we can assume that $\operatorname{ker}\left(\mathbf{A}_{z}^{\prime}\right)$ remains trivial for all $z \in \Gamma^{ \pm}$. The Conley-Zehnder index for $z \in \Gamma^{ \pm}$then computes to (see $[$ Wen16])

$$
\mu_{\mathrm{CZ}}^{\tau}\left(\mathbf{A}_{z}^{\prime}\right)=\mp 1+\mu_{\mathrm{CZ}}^{\tau}\left(\gamma_{z}\right) .
$$

Applying Theorem A.4 and Theorem A.5 we see that $\mathbf{D}_{u}^{\prime}$ is Fredholm with index equal to the right hand side of equation $(22)-2 \# \Gamma$. We then include the dimension of $V$ to obtain the index given by (22).

Corollary A.7. Let $(L, g)$ be a Riemanian manifold and $(X, \omega):=\left(T^{*} L, d \lambda\right)$, seen as a symplectic cobordism with one positive end $M \times[0, \infty)$, where $M=\partial \mathcal{W}_{g}$ is the unit sphere bundle associated to the metric $g$. Let $J_{g}$ be the almost complex structure defined in section 3.1, $\gamma$ a closed geodesic of minimal length in its homology class, and $u_{\gamma, g}$ the unique element of $\mathcal{M}\left(J_{g}, \beta\right)$ asymptotic to $\tilde{\gamma}$ modulo reparametrization. Then

$$
\operatorname{ind}\left(\mathbf{D}_{u_{\gamma, g}}\right)=1
$$

Proof: Recall that $\operatorname{Im} u_{\gamma, g}=\{s \tilde{\gamma}(t) \mid(s, t) \in[0, \infty) \times \mathbb{R} / \ell \mathbb{Z}\}$, where $\tilde{\gamma}(t)=\left(\gamma(t), \dot{\gamma}(t)^{\sharp}\right)$ is the natural lift of $\gamma$ to $M$. Choose an orthonormal basis $\left(\dot{\gamma}(t), e_{2}(t), \ldots, e_{n}(t)\right)$ along $\gamma$ and lift these vectors to vectors $\left(\dot{\tilde{\gamma}}(t), E_{1}(s, t), \ldots, E_{n}(s, t)\right)$, where $E_{j}(s, t)$ is a horizontal vector in $T_{u_{\gamma, g}(s, t)} T^{*} L$. Since $H$ is totally real for the structure $J_{g}$, we get a complex splitting of $T^{*} L$ along $u_{\gamma, g}$ given by

$$
u_{\gamma, g}^{*} T\left(T^{*} L\right)(s, t)=\left\langle R, \frac{\partial}{\partial r}\right\rangle \oplus\left\langle E_{1}(s, t), J_{g} E_{1}(s, t)\right\rangle \oplus \cdots \oplus\left\langle E_{n}(s, t), J_{g} E_{n}(s, t)\right\rangle .
$$

Let $\tau$ be the symplectic trivialization of $u_{\gamma, g}^{*} T\left(T^{*} L\right)$ induced by this decomposition. Then, $c_{1}^{\tau}\left(u_{\gamma, g}^{*} T\left(T^{*} L\right)\right)$ and $\mu^{\tau}\left(u_{\gamma, g}^{*} T\left(T^{*} L\right), T L\right)$ obviously vanish. Since $\chi(D \backslash\{0\})=0$, we get

$$
\operatorname{ind}\left(\mathbf{D}_{u_{\gamma, g}}\right)=\mu_{\mathrm{CZ}}^{\tau}(\tilde{\gamma})+1
$$


It remains to show that $\mu_{\mathrm{CZ}}^{\tau}(\tilde{\gamma})=0$. We recall that this is the Maslov index of the path of symplectic matrices given by the linearization of the Reeb flow (hence the cogeodesic flow) along $\gamma$. Notice that any deformation $g_{\varepsilon}$ of the metric that leaves $\gamma$ a geodesic of fixed length induces a continuous deformation of $\mathbf{D}_{u_{\gamma, g}}$ among Fredholm operators defined on the same Banach space (because $u_{\gamma, g_{\varepsilon}}=u_{\gamma, g}$ for all $\varepsilon$ under this assumption). These Fredholm operators thus all have the same index. By proposition 3.15, completed by remark 3.16, we can therefore assume for our computation of ind $\left(\mathbf{D}_{u_{\gamma, g}}\right)$ that $g$ has the very particular form achieved by proposition 3.15: in Fermi coordinates near $\gamma$ we have $g_{i j}=\left(1+k\left\|x^{\prime}\right\|^{2}\right) \delta_{i j}$. Now for this special metric the derivative of the geodesic flow can be computed explicitly. It preserves the horizontal and the vertical distributions. In the basis $\left(\dot{\gamma}(t), e_{2}(t), \ldots, e_{n}(t)\right)$ of the horizontal distribution it has the form

$$
\left(\begin{array}{cc}
1 & 0 \\
0 & k \mathrm{Id}
\end{array}\right)
$$

The vanishing of the Conley-Zehnder index readily follows.

\section{A.3 A universal moduli space}

Choose an integer $l \geq 2$ and a real number $p>2$, let $k \in\{1, \ldots, l\}$. We define the universal moduli space

$$
\mathcal{M}^{*}\left(\mathcal{O}_{\Gamma}, \mathcal{J}^{l}\right):=\left\{(u, J) \mid \begin{array}{c}
J \in \mathcal{J}^{l}, u \in \mathcal{M}\left(\mathcal{O}_{\Gamma}, J\right) \\
u \text { has an injective point mapped to } \operatorname{int}(K)
\end{array}\right\}
$$

Proposition A.8. The universal moduli space $\mathcal{M}^{*}\left(\mathcal{O}_{\Gamma}, \mathcal{J}^{l}\right)$ is a $C^{l-k}$-Banach submanifold of $\mathcal{B}^{k, p, \delta} \times \mathcal{J}^{l}$.

Proof. The universal moduli space $\mathcal{M}^{*}\left(\mathcal{O}_{\Gamma}, \mathcal{J}^{l}\right)$ is a subset of the zero set of the $C^{l-k}$-section $\mathcal{F}(21)$ of the Banach space bundle $\mathcal{E}^{k-1, p, \delta}$. By showing that the vertical differential $D \mathcal{F}$ is surjective on $\mathcal{M}^{*}\left(\mathcal{O}_{\Gamma}, \mathcal{J}^{l}\right)$, this provides us with the structure of a $C^{l-k}$-Banach submanifold via the infinite dimensional implicit function theorem.

First note that, as in the proof of Proposition 3.2.1 in [MS12], we can give $\mathcal{E}^{k-1, p, \delta}$ the structure of a Banach space bundle of class $C^{l-k}$. The map $\mathcal{F}$ defines a $C^{l-k}$-section of the bundle $\mathcal{E}^{k-1, p, \delta}$ such that the zero set of $\mathcal{F}$ contains $\mathcal{M}^{*}\left(\mathcal{O}_{\Gamma}, \mathcal{J}^{l}\right)$. We now show that the operator $D \mathcal{F}(u, J)$ is surjective for every pair $(u, J) \in \mathcal{M}^{*}\left(\mathcal{O}_{\Gamma}, \mathcal{J}^{l}\right)$.

We prove surjectivity first in the case $k=1$, we consider the operator $D \mathcal{F}(u, J)$ : $T_{u} \mathcal{B}^{1, p, \delta} \times T_{J} \mathcal{J}^{l} \rightarrow L^{p, \delta}\left(\operatorname{Hom}^{0,1}\left(T \dot{\Sigma}, u^{*} T X\right)\right)$, where $D \mathcal{F}(u, J)(\xi, Y)=\mathbf{D}_{u}(\xi)+Y \circ d u \circ j$. By Proposition A.6 the map $\mathbf{D}_{u}$ is Fredholm and thus has a closed range, by a standard result in functional analysis this implies that the range of $D \mathcal{F}(u, J)$ is closed as well. Thus we prove that the image of $D \mathcal{F}(u, J)$ is dense. If the image of $D \mathcal{F}(u, J)$ is not dense, then by the Hahn-Banach theorem, there exists a non-zero section $\eta \in L^{q,-\delta}\left(\operatorname{Hom}^{0,1}\left(T \dot{\Sigma}, u^{*} T X\right)\right)$ 
for $1 / p+1 / q=1$ which annihilates the image of $D \mathcal{F}(u, J)$. In particular this implies

$$
\langle D \mathcal{F}(u, J)(\xi, Y), \eta\rangle_{L^{2}}=0
$$

for every $(\xi, Y) \in T_{u} \mathcal{B}^{1, p, \delta} \times T_{J} \mathcal{J}^{l}$, which in turn implies

$$
\left\langle\mathbf{D}_{u}(\xi), \eta\right\rangle_{L^{2}}=0, \quad\langle Y \circ d u \circ i, \eta\rangle_{L^{2}}=0
$$

for all elements in the domain. In particular this implies that $\eta$ is a weak solution to the formal adjoint equation $\mathbf{D}_{u}^{*}(\eta)=0$. By elliptic regularity $\eta$ is continuous and applying the Carleman similarity principle [MS12] we see that $\eta$ has isolated zeros. Now the set of injective points of $u$ is open, so there exists a $z_{0} \in \dot{\Sigma}$ such that $z_{0}$ is an injective point of $u$ that maps to $\operatorname{int}(K)$ and $\eta\left(z_{0}\right) \neq 0$. By Lemma 3.2.2 of [MS12] there exists a $Y \in T_{J} \mathcal{J}^{l}$ such that $Y \circ d u \circ j\left(z_{0}\right)=\eta\left(z_{0}\right)$, so the inner product $\langle Y \circ d u \circ i, \eta\rangle_{L^{2}}>0$ in some neighborhood of $z_{0}$. Now multiply $Y$ with a non-negative bump function $\beta$ on $X$ which has support in the aforementioned neighborhood. Then we have $\langle(\beta Y) \circ d u \circ i, \eta\rangle_{L^{2}}>0$ since $z_{0}$ is an injective point, which violates (23) and hence surjectivity in the case $k=1$ holds.

In the general case assume $\theta \in W^{k-1, p, \delta}\left(\operatorname{Hom}^{0,1}\left(T \dot{\Sigma}, u^{*} T X\right)\right)$ for $k \geq 2$. Then $\theta \in L^{p, \delta}$ and by surjectivity for $k=1$ there exists $(\xi, Y) \in T_{u} \mathcal{B}^{1, p, \delta} \times T_{J} \mathcal{J}^{l}$ such that $\mathbf{D}_{u}(\xi)+Y \circ$ $d u \circ i=\theta$. $Y$ is of class $C^{l}$, so $Y \circ d u \circ i$ is of class $W^{k-1, p, \delta}$ and thus $\mathbf{D}_{u}(\xi)=\theta-Y \circ d u \circ i$ is of class $W^{k-1, p, \delta}$. Elliptic regularity then implies that $\xi$ is of class $W^{k, p, \delta}$. This proves that $D \mathcal{F}(u, J)$ is surjective for general $k$.

Now, since $\mathbf{D}_{u}$ is a Fredholm operator, by Lemma A.3.6 of [MS12] $D \mathcal{F}(u, J)$ has a right inverse. The infinite dimensional implicit function theorem then implies that $\mathcal{M}^{*}\left(\mathcal{O}_{\Gamma}, \mathcal{J}^{l}\right)$ is a $C^{l-k}$-Banach submanifold of $\mathcal{B}^{k, p, \delta} \times \mathcal{J}^{l}$.

Definition A.9. An almost complex structure $J \in \mathcal{J}_{\text {Cyl }}^{\infty}$ is called regular, if the operator $\mathbf{D}_{u}$ is onto for every $u \in \mathcal{M}^{*}\left(\mathcal{O}_{\Gamma}, J\right)$. We denote by $\mathcal{J}^{\text {reg }} \subset \mathcal{J}_{\text {Cyl }}^{\infty}$ the subset of all regular almost complex structures on $X$.

Proof of Theorem $A .1$ (i). We prove that $\mathcal{M}^{*}\left(\mathcal{O}_{\Gamma}, J\right)$ is a smooth manifold of the prescribed dimension around $u$. Let $J \in \mathcal{J}^{\text {reg }}$ and $u \in \mathcal{M}^{*}\left(\mathcal{O}_{\Gamma}, J\right)$. By lemma $3.7 u$ is smooth. For an integer $k \geq 1$ and $p>2$ we consider the section $\mathcal{F}$ for $J$ fixed in a trivialization of $\mathcal{E}^{k-1, p, \delta}$ over a neighborhood $\mathcal{N}(u)$ of $u \in \mathcal{B}^{k, p, \delta}$. We can identify $\mathcal{N}(u)$ with a neighborhood $U$ of 0 in $T_{u} \mathcal{B}^{k, p, \delta}$ and consider the restricted map

$$
\mathcal{F}_{u}:=\mathcal{F}_{\mid U}: U \longrightarrow W^{k-1, p, \delta}\left(\operatorname{Hom}^{0,1}\left(T \dot{\Sigma},\left(u^{*} T X, J\right)\right)\right) .
$$

Then $\mathcal{F}_{u}$ is a smooth map between Banach spaces and the differential $d \mathcal{F}_{u}(0)=\mathbf{D}_{u}$ is surjective by assumption. By the infinite dimensional implicit function theorem [Sma65], $\mathcal{F}_{u}^{-1}(0)$ intersects a sufficiently small neighborhood of 0 in a smooth finite dimensional submanifold of dimension ind $\left(\mathbf{D}_{u}\right)$. The image of this submanifold under the map $\xi \mapsto \exp _{u}(\xi)$ is a smooth submanifold of $\mathcal{B}^{k, p, \delta}$ that agrees with a neighborhood of $u \in \mathcal{M}^{*}\left(\mathcal{O}_{\Gamma}, J\right)$. Hence $\mathcal{M}^{*}\left(\mathcal{O}_{\Gamma}, J\right)$ is a smooth submanifold of $\mathcal{B}^{k, p, \delta}$ of dimension ind $\left(\mathbf{D}_{u}\right)$ locally around $u$. 
Proposition A.10. The projection $\mathcal{M}^{*}\left(\mathcal{O}_{\Gamma}, \mathcal{J}^{l}\right) \rightarrow \mathcal{J}^{l}$ is a nonlinear Fredholm map and for $l$ large enough the set of regular values in $\mathcal{J}^{l}$ is dense.

Proof. The projection $\pi: \mathcal{M}^{*}\left(\mathcal{O}_{\Gamma}, \mathcal{J}^{l}\right) \rightarrow \mathcal{J}^{l}$ is, by Proposition A.8 for $k=1$, a $C^{l-1}$-map between separable $C^{l-1}$-Banach manifolds. The tangent space at $(u, J)$

$$
T_{(u, J)} \mathcal{M}^{*}\left(\mathcal{O}_{\Gamma}, \mathcal{J}^{l}\right) \subset T_{u} \mathcal{B}^{1, p, \delta} \times T_{J} \mathcal{J}^{l},
$$

consists of all pairs $(\xi, Y)$ such that $\mathbf{D}_{u}(\xi)+Y(u) \circ d u \circ j=0$. The derivative $d \pi(u, J)$ is just the projection $(\xi, Y) \mapsto Y$. Thus the kernel of $d \pi(u, J)$ is isomorphic to the kernel of $\mathbf{D}_{u}$. By a standard result in functional analysis [MS12, Lemma A.3.6] the cokernel of $d \pi(u, J)$ is also isomorphic to the cokernel of $\mathbf{D}_{u}$. It follows that $d \pi(u, J)$ is a Fredholm operator with the same index as $\mathbf{D}_{u}$. Moreover the operator $d \pi(u, J)$ is onto precisely when $\mathbf{D}_{u}$ is onto. This implies that a regular value $J$ of $\pi$ is an almost complex structure with the property that $\mathbf{D}_{u}$ is onto for every somewhere injective curve $u \in \mathcal{M}^{*}\left(\mathcal{O}_{\Gamma}, J\right)=\pi^{-1}(J)$. In other words,

$$
\mathcal{J}^{\mathrm{reg}, l}:=\left\{J \in \mathcal{J}^{l} \mid \mathbf{D}_{u} \text { is onto for all } u \in \mathcal{M}^{*}\left(\mathcal{O}_{\Gamma}, J\right)\right\}
$$

the set of regular almost complex structures of class $C^{l}$ is the set of regular values of $\pi$. By the Sard-Smale theorem [Sma65], this set is of second category in the sense of Baire. Here we use the fact that $\mathcal{M}^{*}\left(\mathcal{O}_{\Gamma}, \mathcal{J}^{l}\right)$ and the projection $\pi$ are of class $C^{l-1}$ and we can apply Sard-Smale whenever $l-2 \geq \operatorname{ind}\left(\mathbf{D}_{u}\right)$. Thus the set $\mathcal{J}^{\text {reg, } l}$ is dense in $\mathcal{J}^{l}$ with respect to the $C^{l}$-topology for $l$ sufficiently large.

Proof of Theorem A.1 (ii). We must show that the set $\mathcal{J}^{\text {reg }}$ is of second category in $\mathcal{J}_{\text {Cyl }}^{\infty}$.

We fix metrics on $X$ and $\dot{\Sigma}$ that are translation invariant on the cylindrical ends and we denote by $\operatorname{dist}(\cdot, \cdot)$ the induced distance functions. For $N \in \mathbb{N}$ consider the set

$$
\mathcal{J}_{N}^{\text {reg }}:=\mathcal{J}_{N}^{\text {reg }}\left(\mathcal{O}_{\Gamma}\right) \subset \mathcal{J}_{\text {Cyl }}^{\infty}
$$

of all smooth almost complex structures $J$ such that the operator $\mathbf{D}_{u}$ is onto for every $J$-holomorphic curve $u \in \mathcal{M}^{*}\left(\mathcal{O}_{\Gamma}, J\right)$ that satisfies

(i) $\sup _{z \in \dot{\Sigma}}|d u(z)| \leq N$;

(ii) there exists a $z_{0} \in \dot{\Sigma}$ such that

$$
\operatorname{dist}\left(u\left(z_{0}\right), X \backslash \operatorname{int}(K)\right) \geq \frac{1}{N}, \quad\left|d u\left(z_{0}\right)\right| \geq \frac{1}{N}, \quad \inf _{z \in \dot{\Sigma} \backslash\left\{z_{0}\right\}} \frac{\operatorname{dist}\left(u\left(z_{0}\right), u(z)\right)}{\operatorname{dist}\left(z_{0}, z\right)} \geq \frac{1}{N} .
$$

Note that the set of such $u$ has a somewhere injective point mapped to int $(K)$. Furthermore, every asymptotically cylindrical $J$-holomorphic curve with an injective point mapped to $\operatorname{int}(K)$ satisfies these conditions for some value of $N \in \mathbb{N}$.

We claim that $\mathcal{J}_{N}^{\text {reg }}$ is open and dense in $\mathcal{J}_{\text {Cyl }}^{\infty}$. We first show that this set is open, which is equivalent to the complement being closed. Assume we have a sequence $J_{\nu} \notin \mathcal{J}_{N}^{\text {reg }}$, 
$J_{\nu} \rightarrow J$ in $C^{\infty}$. This means that for every $\nu$ there exists a $J_{\nu}$-holomorphic $u_{\nu}$ and a $z_{\nu} \in \dot{\Sigma}$ that satisfy conditions (i) and (ii) and such that $\mathbf{D}_{u_{\nu}}$ is not surjective. Since the first derivatives of $u_{\nu}$ are uniformly bounded and (ii) is a closed condition, by a standard elliptic bootstrapping argument [MS12, Theorem B.4.2] there exists a subsequence $u_{\nu_{i}}$ that converges uniformly with all derivatives to a smooth $J$-holomorphic curve $u$ that satisfies conditions (i) and (ii). Since the operators $\mathbf{D}_{u_{\nu_{i}}}$ are not surjective, it follows that $\mathbf{D}_{u}$ is not surjective either. This shows that $J \notin \mathcal{J}_{N}^{\text {reg }}$ and thus $\mathcal{J}_{N}^{\text {reg }}$ is open in the $C^{\infty}$-topology. We now prove that $\mathcal{J}_{N}^{\text {reg }}$ is dense in $\mathcal{J}_{\text {Cyl }}^{\infty}$. Let $\mathcal{J}_{N}^{\text {reg, }, l} \subset \mathcal{J}^{l}$ be the set of all $J \in \mathcal{J}^{l}$ such that the operator $\mathbf{D}_{u}$ is onto for every $u \in \mathcal{M}^{*}\left(\mathcal{O}_{\Gamma}, J\right)$ that satisfies conditions (i) and (ii). Now note that

$$
\mathcal{J}_{N}^{\mathrm{reg}}=\mathcal{J}_{N}^{\mathrm{reg}, l} \cap \mathcal{J}_{\mathrm{Cyl}}^{\infty} \text {. }
$$

Let $J \in \mathcal{J}_{\text {Cyl }}^{\infty} \subset \mathcal{J}^{l}$. Now by Proposition A.10 the set $\mathcal{J}^{\text {reg,l}}$ of (24) is dense in $\mathcal{J}^{l}$ for large $l$, so there exists a sequence $J_{l} \in \mathcal{J}^{\text {reg, } l}$ such that

$$
\left\|J_{l}-J\right\|_{C^{l}} \leq 2^{-l}
$$

Since $J_{l} \in \mathcal{J}_{N}^{\text {reg,l}}$ and $\mathcal{J}_{N}^{\text {reg,l}}$ is open in the $C^{l}$-topology, there exists an $\varepsilon_{l}$ such that for every $J^{\prime} \in \mathcal{J}^{l}$

$$
\left\|J_{l}-J^{\prime}\right\|_{C^{l}}<\varepsilon_{l} \Longrightarrow J^{\prime} \in \mathcal{J}_{N}^{\mathrm{reg}, l} .
$$

Choose $J_{l}^{\prime} \in \mathcal{J}_{\mathrm{Cyl}}^{\infty}$ to be any smooth element such that

$$
\left\|J_{l}^{\prime}-J_{l}\right\|_{C^{l}} \leq \min \left\{\varepsilon_{l}, 2^{-l}\right\} .
$$

Then $J_{l}^{\prime} \in \mathcal{J}_{N}^{\text {reg, } l} \cap \mathcal{J}_{\text {Cyl }}^{\infty}=\mathcal{J}_{N}^{\text {reg }}$ converges to $J$ in the $C^{\infty}$-topology. This shows that the set of $\mathcal{J}_{N}^{\text {reg }}$ is dense in $\mathcal{J}_{\text {Cyl }}^{\infty}$ as claimed. Thus $\mathcal{J}^{\text {reg }}$ is the intersection of a countable number of open dense sets $\mathcal{J}_{N}^{\text {reg }}, N \in \mathbb{N}$, and is so of second category.

Proof of Theorem A.2. Applying the ideas of the proof of Theorem A.1 (i) to the bundle $\mathcal{E}^{k-1, p, \delta} \rightarrow \mathcal{B}^{k, p, \delta} \times[0,1]$ gives us the manifold structure of $\mathcal{W}^{*}\left(\mathcal{O}_{\Gamma},\left\{J_{t}\right\}\right)$. For (ii) we proceed analogous to the proof of Theorem A.1. Define $\mathcal{J}^{l}\left(J_{0}, J_{1}\right)$ to be the space of $C^{l}$-homotopies in $\mathcal{J}^{l}$ from $J_{0}$ to $J_{1}$. Consider the universal moduli space $\mathcal{W}^{*}\left(\mathcal{O}_{\Gamma}, \mathcal{J}^{l}\left(J_{0}, J_{1}\right)\right)$. One can show that this space is a $C^{l-1}$-Banach manifold and that the projection map to $\mathcal{J}^{l}\left(J_{0}, J_{1}\right)$ is a $C^{l-1}$ Fredholm map. By Sard-Smale, the regular values of this map is dense for $l$ sufficiently large and produces the desired subset of regular homotopies. The conclusion to the case of smooth homotopies is then as in the proof of Theorem A.1.

\section{References}

[Abb14] C. Abbas. An introduction to compactness results in symplectic field theory. Springer, Heidelberg, 2014.

[Abo11] M. Abouzaid. A cotangent fibre generates the Fukaya category. Adv. Math., 228(2):894-939, 2011.

[Abr70] R. Abraham. Bumpy metrics. In Global Analysis (Proc. Sympos. Pure Math., Vol. XIV, Berkeley, Calif., 1968), pages 1-3. Amer. Math. Soc., Providence, R.I., 1970.

[Ano82] D. V. Anosov. Generic properties of closed geodesics. Izv. Akad. Nauk SSSR Ser. Mat., 46(4):675709, 896, 1982. 
[AOO16] L. Amorim, Y.-G. Oh, and J. Oliveira dos Santos. Exact Lagrangian submanifolds, Lagrangian spectral invariants and Aubry-Mather theory. ArXiv e-prints, March 2016.

[AS06] A. Abbondandolo and M. Schwarz. On the Floer homology of cotangent bundles. Comm. Pure Appl. Math., 59(2):254-316, 2006.

[BCG95] G. Besson, G. Courtois, and S. Gallot. Entropies et rigidités des espaces localement symétriques de courbure strictement négative. Geom. Funct. Anal., 5(5):731-799, 1995.

$\left[\mathrm{BEH}^{+} 03\right]$ F. Bourgeois, Y. Eliashberg, H. Hofer, K. Wysocki, and E. Zehnder. Compactness results in symplectic field theory. Geom. Topol., 7:799-888, 2003.

[BEP12] L. Buhovsky, M. Entov, and L. Polterovich. Poisson brackets and symplectic invariants. Selecta Math. (N.S.), 18(1):89-157, 2012.

[BHS16] L. Buhovsky, V. Humilière, and S. Seyfaddini. A $\mathrm{C}^{\wedge} 0$ counterexample to the Arnold conjecture. ArXiv e-prints, September 2016.

[BO16] L. Buhovsky and E. Opshtein. Some quantitative results in $\mathcal{C}^{0}$ symplectic geometry. Invent. Math., 205(1):1-56, 2016.

[Cha06] I. Chavel. Riemannian Geometry: A Modern Introduction. Cambridge Studies in Advanced Mathematics. Cambridge University Press, 2006.

[Che98] Y. V. Chekanov. Lagrangian intersections, symplectic energy, and areas of holomorphic curves. Duke Math. J., 95(1):213-226, 1998.

[CK94] C. B. Croke and B. Kleiner. Conjugacy and rigidity for manifolds with a parallel vector field. J. Differential Geom., 39(3):659-680, 1994.

[CM18] K. Cieliebak and K. Mohnke. Punctured holomorphic curves and Lagrangian embeddings. Invent. Math., 212(1):213-295, 2018.

[Cou14] S. Courte. Contact manifolds with symplectomorphic symplectizations. Geom. Topol., 18(1):1-15, 2014.

[Dra04] D. L. Dragnev. Fredholm theory and transversality for noncompact pseudoholomorphic maps in symplectizations. Comm. Pure Appl. Math., 57(6):726-763, 2004.

[DRGI16] G. Dimitroglou Rizell, E. Goodman, and A. Ivrii. Lagrangian isotopy of tori in $S^{2} \times S^{2}$ and $\mathbb{C} P^{2}$. Geom. Funct. Anal., 26(5):1297-1358, 2016.

[EGM16] M. Entov, Y. Ganor, and C. Membrez. Lagrangian isotopies and symplectic function theory. ArXiv e-prints, October 2016.

[Elı̌67] H. I. Elı̆asson. Geometry of manifolds of maps. J. Differential Geometry, 1:169-194, 1967.

[Gri98] A. A. Grigor'yants. On an interpretation of the torsion tensor of a connection. Izv. Vyssh. Uchebn. Zaved. Mat., (8):22-28, 1998.

[Gro99] M. Gromov. Metric structures for Riemannian and non-Riemannian spaces, volume 152 of Progress in Mathematics. Birkhäuser Boston, Inc., Boston, MA, 1999. Based on the 1981 French original [ MR0682063 (85e:53051)], With appendices by M. Katz, P. Pansu and S. Semmes, Translated from the French by Sean Michael Bates.

[Gut12] J. Gutt. The Conley-Zehnder index for a path of symplectic matrices. ArXiv e-prints, 2012.

[HLS15] V. Humilière, R. Leclercq, and S. Seyfaddini. Coisotropic rigidity and $C^{0}$-symplectic geometry. Duke Math. J., 164(4):767-799, 2015.

[HLS16] V. Humilière, R. Leclercq, and S. Seyfaddini. Reduction of symplectic homeomorphisms. Ann. Sci. Éc. Norm. Supér. (4), 49(3):633-668, 2016.

[HWZ96] H. Hofer, K. Wysocki, and E. Zehnder. Properties of pseudoholomorphic curves in symplectisations. I. Asymptotics. Ann. Inst. H. Poincaré Anal. Non Linéaire, 13(3):337-379, 1996.

[HWZ99] H. Hofer, K. Wysocki, and E. Zehnder. Properties of pseudoholomorphic curves in symplectizations. III. Fredholm theory. In Topics in nonlinear analysis, volume 35 of Progr. Nonlinear Differential Equations Appl., pages 381-475. Birkhäuser, Basel, 1999. 
[IS10] S. Ivashkovich and A. Sukhov. Schwarz reflection principle, boundary regularity and compactness for J-complex curves. Ann. Inst. Fourier (Grenoble), 60(4):1489-1513, 2010.

[Kra11] T. Kragh. Parametrized Ring-Spectra and the Nearby Lagrangian Conjecture. ArXiv e-prints, July 2011.

[LS94] F. Laudenbach and J.-C. Sikorav. Hamiltonian disjunction and limits of Lagrangian submanifolds. Internat. Math. Res. Notices, (4):161 ff., approx. 8 pp. (electronic), 1994.

[MS95] D. McDuff and D. Salamon. Introduction to symplectic topology. Oxford Mathematical Monographs. The Clarendon Press Oxford University Press, New York, 1995. Oxford Science Publications.

[MS12] D. McDuff and D. Salamon. J-holomorphic curves and symplectic topology, volume 52 of American Mathematical Society Colloquium Publications. American Mathematical Society, Providence, RI, second edition, 2012.

[MW95] M. J. Micallef and B. White. The structure of branch points in minimal surfaces and in pseudoholomorphic curves. Ann. of Math. (2), 141(1):35-85, 1995.

[Ops09] E. Opshtein. $\mathcal{C}^{0}$-rigidity of characteristics in symplectic geometry. Ann. Sci. Éc. Norm. Supér. (4), 42(5):857-864, 2009.

[PPS03] G. P. Paternain, L. Polterovich, and K. F. Siburg. Boundary rigidity for Lagrangian submanifolds, non-removable intersections, and Aubry-Mather theory. Mosc. Math. J., 3(2):593-619, 745, 2003. Dedicated to Vladimir I. Arnold on the occasion of his 65th birthday.

[Sal99] D. Salamon. Lectures on Floer homology. In Symplectic geometry and topology (Park City, UT, 1997), volume 7 of IAS/Park City Math. Ser., pages 143-229. Amer. Math. Soc., Providence, RI, 1999.

[Sch95] M. Schwarz. Cohomology operations from $S^{1}$-cobordisms in Floer homology. PhD thesis, E.T.H. Zurich, 1995.

[Sik89] J.-C. Sikorav. Rigidité symplectique dans le cotangent de $T^{n}$. Duke Math. J., 59(3):759-763, 1989.

[Sma65] S. Smale. An infinite dimensional version of Sard's theorem. Amer. J. Math., 87:861-866, 1965.

[SW06] D. A. Salamon and J. Weber. Floer homology and the heat flow. Geom. Funct. Anal., 16(5):10501138, 2006.

[Wen10] C. Wendl. Automatic transversality and orbifolds of punctured holomorphic curves in dimension four. Comment. Math. Helv., 85(2):347-407, 2010.

[Wen16] C. Wendl. Lectures on Symplectic Field Theory. ArXiv e-prints, December 2016.

[Zeh13] K. Zehmisch. The codisc radius capacity. Electron. Res. Announc. Math. Sci., 20:77-96, 2013.

Cedric Membrez

School of Mathematical Sciences

Tel Aviv University

Tel Aviv 69978, Israel

ckmembrez@gmail.com
Emmanuel Opshtein, Institut de Recherche Mathématique Avancée UMR 7501, Université de Strasbourg et CNRS 7 rue René Descartes 67000 Strasbourg, France

opshtein@unistra.fr 